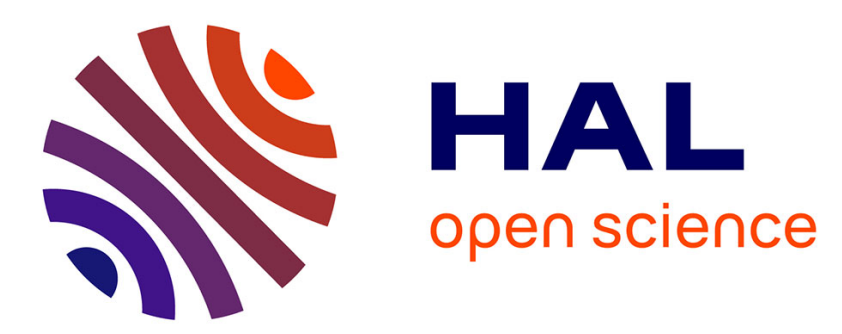

\title{
Reconstruction of Full Rank Algebraic Branching Programs
}

Neeraj Kayal, Vineet Nair, Chandan Saha, Sébastien Tavenas

\section{To cite this version:}

Neeraj Kayal, Vineet Nair, Chandan Saha, Sébastien Tavenas. Reconstruction of Full Rank Algebraic Branching Programs. ACM Transactions on Computation Theory, 2019, 11 (1), pp.1-56. $10.1145 / 3282427$. hal-02408621

\section{HAL Id: hal-02408621 \\ https://hal.science/hal-02408621}

Submitted on 13 Dec 2019

HAL is a multi-disciplinary open access archive for the deposit and dissemination of scientific research documents, whether they are published or not. The documents may come from teaching and research institutions in France or abroad, or from public or private research centers.
L'archive ouverte pluridisciplinaire HAL, est destinée au dépôt et à la diffusion de documents scientifiques de niveau recherche, publiés ou non, émanant des établissements d'enseignement et de recherche français ou étrangers, des laboratoires publics ou privés. 


\title{
Reconstruction of Full Rank Algebraic Branching Programs
}

\author{
Neeraj Kayal ${ }^{1}$, Vineet Nair ${ }^{2}$, Chandan $\mathrm{Saha}^{3}$, and \\ Sébastien Tavenas*4
}

1 Microsoft Research India, Bengaluru, India

neeraka@microsoft.com

2 Indian Institute of Science, Bengaluru, India

vineet.nair@csa.iisc.ernet.in

3 Indian Institute of Science, Bengaluru, India

chandan@csa.iisc.ernet.in

4 Université Savoie Mont Blanc, CNRS, LAMA, Chambéry, France

sebastien.tavenas@univ-smb.fr

\begin{abstract}
An algebraic branching program (ABP) A can be modelled as a product expression $X_{1} \cdot X_{2} \ldots X_{d}$, where $X_{1}$ and $X_{d}$ are $1 \times w$ and $w \times 1$ matrices respectively, and every other $X_{k}$ is a $w \times w$ matrix; the entries of these matrices are linear forms in $m$ variables over a field $\mathbb{F}$ (which we assume to be either $\mathbb{Q}$ or a field of characteristic poly $(m)$ ). The polynomial computed by $\mathrm{A}$ is the entry of the $1 \times 1$ matrix obtained from the product $\prod_{k=1}^{d} X_{k}$. We say A is a full rank ABP if the $w^{2}(d-2)+2 w$ linear forms occurring in the matrices $X_{1}, X_{2}, \ldots, X_{d}$ are $\mathbb{F}$-linearly independent. Our main result is a randomized reconstruction algorithm for full rank ABPs: Given blackbox access to an $m$-variate polynomial $f$ of degree at most $m$, the algorithm outputs a full rank ABP computing $f$ if such an ABP exists, or outputs 'no full rank ABP exists' (with high probability). The running time of the algorithm is polynomial in $m$ and $\beta$, where $\beta$ is the bit length of the coefficients of $f$. The algorithm works even if $X_{k}$ is a $w_{k-1} \times w_{k}$ matrix (with $w_{0}=w_{d}=1$ ), and $\mathbf{w}=\left(w_{1}, \ldots, w_{d-1}\right)$ is unknown.

The result is obtained by designing a randomized polynomial time equivalence test for the family of iterated matrix multiplication polynomial $\mathrm{IMM}_{\mathbf{w}, d}$, the $(1,1)$-th entry of a product of $d$ rectangular symbolic matrices whose dimensions are according to $\mathbf{w} \in \mathbb{N}^{d-1}$. At its core, the algorithm exploits a connection between the irreducible invariant subspaces of the Lie algebra of the group of symmetries of a polynomial $f$ that is equivalent to $\mathrm{IMM}_{\mathbf{w}, d}$ and the 'layer spaces' of a full rank ABP computing $f$. This connection also helps determine the group of symmetries of $\mathrm{IMM}_{\mathbf{w}, d}$ and show that $\mathrm{IMM}_{\mathbf{w}, d}$ is characterized by its group of symmetries.
\end{abstract}

1998 ACM Subject Classification F.1.3 Complexity Measures and Classes

Keywords and phrases Circuit reconstruction, algebraic branching programs, equivalence test, iterated matrix multiplication, Lie algebra

Digital Object Identifier 10.4230/LIPIcs.CCC.2017.21

* A part of this work was done during a postdoctoral stay in Microsoft Research India.

\footnotetext{
(c) (i) () Neeraj Kayal, Vineet Nair, Chandan Saha, and Sébastien Tavenas; licensed under Creative Commons License CC-BY 32nd Computational Complexity Conference (CCC 2017). Editor: Ryan O'Donnell; Article No. 21; pp. 21:1-21:61 Leibniz International Proceedings in Informatics LI I ICS Schloss Dagstuhl - Leibniz-Zentrum für Informatik, Dagstuhl Publishing, Germany

(e) COMPUTATIONAL COMPLEXITY CONFERENCE 


\section{Introduction}

\subsection{Circuit reconstruction}

Reconstruction of arithmetic circuits is the algebraic analogue of exact learning [5] of Boolean circuits using membership and equivalence queries. A reconstruction algorithm takes input an oracle access to an $m$-variate degree $d$ polynomial $f$ computed by a size $s$ arithmetic circuit from some circuit class $\mathcal{C}$, and outputs an arithmetic circuit (preferably from the same class) of not too large size ${ }^{1}$ computing $f$. The algorithm is allowed to make two kinds of adaptive queries to the oracle: It may ask for evaluation of $f$ at a point $\mathbf{a} \in \mathbb{F}^{m}$ chosen by the algorithm (membership query). It may also form a circuit $C$ (a hypothesis) and ask if the polynomial $g$, computed by $C$, equals $f$; if not, the oracle returns a point $\mathbf{b} \in \mathbb{F}^{m}$ such that $f(\mathbf{b}) \neq g(\mathbf{b})$ (equivalence query) ${ }^{2}$. The desired running time of the algorithm is polynomial in $m, d, s$ and the bit length of the coefficients of $f$.

Circuit reconstruction is a natural learning problem in algebraic complexity theory and is closely related to two other fundamental problems, lower bound and polynomial identity testing. Building on the ideas in [21, 2] and [28], Volkovich [42] showed that a polynomial time reconstruction algorithm for a circuit class $\mathcal{C}$ can be used to compute an $m$-variate multilinear polynomial $h$ in $2^{O(m)}$ time such that any circuit from $\mathcal{C}$ computing $h$ has size $2^{\Omega(m) 3}$. Also, an efficient reconstruction algorithm (that uses only membership queries) for a class of circuits automatically gives an efficient blackbox ${ }^{4}$ identity testing algorithm for the same class. In this sense, reconstruction is a 'harder' problem than lower bound and identity testing ${ }^{5}$. However, if we allow reconstruction algorithms to be randomized (thereby giving them the power of identity testing) then we can hope to have efficient reconstructions even for some classes of circuits for which efficient blackbox identity testing algorithms are not known yet. Indeed, a randomized polynomial time reconstruction algorithm for read-once oblivious algebraic branching programs (ROABP) was given in [29] much before the quasi-polynomial time hitting-set generators for the same model were designed [14, 3]. The case of read-once formulas is similar (see [39]). A randomized reconstruction algorithm need not use equivalence queries as a random point $\mathbf{b}$ is a witness for $f(\mathbf{b}) \neq g(\mathbf{b})$, if $f \neq g^{6}$. In this article, we will assume that reconstruction algorithms use only membership queries, unless we mention equivalence queries explicitly.

Another way to moderate the reconstruction setup is given by average-case reconstruction. Here the input polynomial $f$ is picked according to some 'natural' distribution on circuits from a class $\mathcal{C}$. This relaxation led to the development of randomized polynomial time reconstruction algorithm for some powerful circuit classes [17, 19] (albeit on average), including arithmetic formulas for which we do not know of any super-polynomial lower bound. The notion of average-case reconstruction is related to pseudo-random polynomial

1 We allow the algorithm to output sub-optimal size circuit as it is NP-hard to compute an optimal circuit for $f$ even for restricted classes like set-multilinear depth three circuits [20].

2 Throughout this article we will assume that the base field $\mathbb{F}$ is sufficiently large, so if $f(\mathbf{b})=g(\mathbf{b})$ for every $\mathbf{b} \in \mathbb{F}^{m}$ then $f=g$.

3 Such an implication is not known for an $h$ belonging to a VNP family.

4 The algorithm has blackbox access to $f$, i.e. it can make only membership queries to an oracle holding $f$.

5 Not much is known about the reverse direction: Do strong lower bounds or efficient blackbox identity testing for a circuit class imply efficient reconstruction for the same class? For certain interesting circuit classes, the techniques used for identity testing and lower bounds do help in efficient reconstruction (see $[39,17])$.

6 The algorithm in [29] is deterministic if we allow equivalence queries. 
families ${ }^{7}$ and the prospects/limitations of lower bound proofs: An efficient reconstruction algorithm for polynomials generated according to a distribution $\mathrm{D}$ on circuits from class $\mathcal{C}$ implies that $D$ does not generate a pseudo-random polynomial family. Such an algorithm gives evidence (contingent on the extent of naturalness of $\mathrm{D}$ ) that most circuits in $\mathcal{C}$ have sufficient "structural/mathematical" properties in them that the reconstruction algorithm is able to exploit efficiently to distinguish polynomials computed by them from random polynomials. This may hint at an intriguing possibility that $\mathcal{C}$ is adequately 'weak' and amenable to explicit lower bound proofs against it. On the contrary, if D does generate a pseudo-random polynomial family then certain widely used strategies to prove lower bounds will not work for $\mathcal{C}$, much like natural proofs for Boolean circuits [36] (see the discussion in $[1])$.

\section{Previous work on reconstruction}

We will assume that a circuit from class $\mathcal{C}$ computing the input polynomial $f$ has a sum gate at the output. Otherwise, we can apply the factorization algorithm in [22] to gain blackbox access to all the irreducible factors of $f$, thereby reducing the problem to a potentially simpler class of circuits at the cost of making the reconstruction algorithm randomized. Thus, depth two, depth three and depth four circuits would mean $\Sigma \Pi, \Sigma \Pi \Sigma$ and $\Sigma \Pi \Sigma \Pi$ circuits respectively.

Low depth circuits: A polynomial time reconstruction algorithm for depth two circuits follows from the sparse polynomial interpolation algorithm in [30]. By analysing the rank of the partial derivatives matrix, Klivans and Shpilka [29] gave a randomized reconstruction algorithm ${ }^{8}$ for depth three circuits with fan-in of every product gate bounded by $d$ in time polynomial in the size of the circuit and $2^{d}$. Prior to this, a polynomial time randomized reconstruction algorithm for set-multilinear depth three circuits followed from [7]. In both [29] and [7] the output hypothesis is an ROABP. For depth three circuits with two product gates, Shpilka [37] gave a randomized reconstruction algorithm over a finite field $\mathbb{F}$ that has running time quasi-polynomial ${ }^{9}$ in $m, d$ and $|\mathbb{F}|$. This algorithm was derandomized and extended to depth three circuits with constant number of product gates in [23]. The output hypothesis in [37] is a depth three circuit with two product gates (unless the circuit has a low simple $\operatorname{rank}^{10}$ ), but it works only over finite fields. Recently, Sinha [40] gave a polynomial time randomized reconstruction algorithm for depth three circuits with two product gates over rationals ${ }^{11}$; the output of Sinha's algorithm is also a depth three circuit with two product gates (unless the simple rank of the circuit is less than a fixed constant). For multilinear depth four circuits with two top level product gates, [18] gave a randomized polynomial time reconstruction algorithm that works over both finite fields and rationals.

Restricted formulas and ABP: Recently, Minahan and Volkovich [33] gave a polynomial time reconstruction algorithm for read-once formulas by strengthening the analysis in [38], the

\footnotetext{
7 Intuitively, a distribution D on $m$-variate degree- $d$ polynomials using a random seed of length $s=$ $(m d)^{O(1)}$ generates a pseudo-random polynomial family if any algorithm that distinguishes polynomials coming from $\mathrm{D}$ from uniformly-random $m$-variate degree- $d$ polynomials with a non-negligible bias, takes time exponential in $s$.

8 The algorithm is deterministic if equivalence queries are used.

${ }^{9}$ The running time is polynomial in $m,|\mathbb{F}|$ if the depth three circuit is additionally multilinear.

${ }^{10}$ The dimension of the span of the linear forms in the two gates after removing their gcd.

${ }^{11}$ The result holds over characteristic zero fields. We state it for rationals as bit complexity concerns us.
} 
latter has a quasi-polynomial time reconstruction algorithm for the same model. Forbes and Shpilka [14] gave quasi-polynomial time reconstruction algorithms for ROABP, set-multilinear $\mathrm{ABP}$ and non-commutative ABP by derandomizing ${ }^{12}$ the algorithm in [29]. Prior to this, the case of non-commutative ABP reconstruction was solved in [6] assuming blackbox access to the internal gates of the input ABP.

Average-case reconstruction: Few reconstruction algorithms are known under distributional assumptions on the inputs. Gupta, Kayal and Lokam [17] gave a randomized polynomial time reconstruction algorithm for random multilinear formulas picked from a natural distribution: every sum gate computes a random linear combinations of its two children (subformulas), and at every product gate the set of variables is partitioned randomly into two equal size sets between its two children (subformulas); the subformulas are then constructed recursively. In [19], a randomized polynomial time reconstruction algorithm was given for random formulas picked from the distribution of size $s$ complete binary trees with alternating layers of sum and product gates, and the linear forms at the leaves are chosen independently and uniformly at random.

\subsection{Motivation and model}

Motivation: Given the results in $[17,19]$, it is natural to study the complexity of average-case reconstruction for models more powerful than formulas, like ABPs. Another motivation is the following: Aaronson [1] gave a candidate for pseudo-random family of low degree polynomials over a finite field $\mathbb{F}$. There it is conjectured that the family $\left\{\operatorname{Det}_{d}(A \cdot \mathbf{x}): A \in \mathbb{F}^{2} \times m\right\}$, where Det $_{d}$ is the determinant of a $d \times d$ symbolic matrix and $|\mathbf{x}|=m$, is pseudo-random if $A$ is chosen uniformly at random from $\mathbb{F}^{d^{2} \times m}$ and $m \ll d$. If this is shown to be true (under plausible hardness assumptions) then that would demonstrate a natural-proofs-like barrier in the algebraic world. Although the conjecture is made for finite fields, it remains interesting to study even if the entries of $A$ are chosen from a large enough subset of $\mathbb{Q}$ (or char $(\mathbb{F})>d^{c}$ for a sufficiently large constant $c$ ). Moreover, since determinant is complete (under p-projections) for algebraic branching programs [32] and so is $\mathrm{IMM}_{w, d}$ - the $(1,1)$-th entry of a product of $d w \times w$ symbolic matrices - it is natural to ask if $\left\{\operatorname{IMM}_{w, d}(A \cdot \mathbf{x}): A \in \mathbb{F}^{n \times m}\right\}$ is also a pseudo-random polynomial family when $A$ is random and $m \ll w^{2} d$; here $n=w^{2}(d-2)+2 w$ is the number of variables in $\mathrm{IMM}_{w, d}$. If yes then we cannot hope to design an efficient reconstruction algorithm for algebraic branching programs in the average-case. On the other hand, if such an average-case reconstruction is possible then the above family generated by linear projections of $\mathrm{IMM}_{w, d}$ is not pseudo-random. This motivates us to pose Problem 3 below (rather optimistically), and study a couple of special cases when it can be solved - one is in this article and the other in an upcoming work $[27]^{13}$.

Algebraic branching program: Algebraic branching program (ABP), an arithmetic analogue of Boolean branching program, is a well-studied model in algebraic complexity theory specially because it captures the complexity of polynomials like the iterated matrix multiplication and the symbolic determinant. Separating the computational powers of formulas and ABPs, and that of ABPs and circuits are outstanding open problems in arithmetic circuit complexity.

\footnotetext{
${ }^{12}$ Replacing the equivalence queries by quasi-polynomial size hitting-sets for ROABP.

${ }^{13}$ See Section 1.4 for some details on this work.
} 
An $\mathrm{ABP}$ is defined below. For the rest of this article, the base field $\mathbb{F}$ would be the field of rationals $\mathbb{Q}^{14}$.

- Definition 1 (Algebraic branching program). An algebraic branching program (ABP) of width $w$ and length $d$ is a product expression $X_{1} \cdot X_{2} \ldots X_{d}$, where $X_{1}, X_{d}$ are row and column vectors of length $w$ respectively, and for $k \in[2, d-1], X_{k}$ is a $w \times w$ matrix. The entries in $X_{1}$ to $X_{d}$ are affine forms in the variables $\mathbf{x}=\left\{x_{1}, x_{2}, \ldots, x_{m}\right\}$. The polynomial computed by the ABP is the entry of the $1 \times 1$ matrix obtained from the product $\prod_{k=1}^{d} X_{k}$. An ABP of width $w$, length $d$, and in $m$ variables, and with the coefficients of the affine forms from $S \subseteq \mathbb{F}$, will be called a $(w, d, m, S)$-ABP.

An alternate definition: Alternatively, an ABP is defined as a layered directed acyclic graph with a source $s$ and a sink $t$. A width $w$ and length $d$ ABP has $d+1$ layers, where the first and the last layers contain one vertex each, labelled $s$ and $t$ respectively, and every other layer has $w$ vertices. There is an edge from every vertex in layer $k$ to every vertex in layer $k+1$, for all $k \in[d]$, and these edges between adjacent layers are labelled by affine forms in $\mathbf{x}$ variables. The weight of a path from $s$ to $t$ is the product of the edge labels in the path, and the polynomial computed by the ABP is the sum of the weights of all paths from $s$ to $t$. It is easy to verify that the two definitions of ABP are equivalent. We use either of these definitions in our arguments later based on suitability.

Average-case ABP reconstruction: In order to study average-case complexity of the reconstruction problem for ABPs, we need to define a distribution on polynomials computed by ABPs. A seemingly natural distribution is as follows: Consider the universe of all polynomials computed by $(w, d, m, S)$-ABPs for some finite set $S \subseteq \mathbb{F}$ of large enough size. Pick a polynomial $f$ uniformly at random from this universe, and give blackbox access to $f$ as input to a reconstruction algorithm. However, a distribution is 'realistic' only if there is an efficient sampling algorithm that outputs (some suitable circuit representation of) $f$ according to the distribution. For the above distribution, it is not clear if such an efficient sampling algorithm exists. A reason being, multiple different ABPs may be computing the same polynomial, so picking a random $\mathrm{ABP}$ is not sufficient to sample from this distribution. However, picking a random $\mathrm{ABP}$ (as described below) gives another natural distribution for which there is a trivial efficient sampling algorithm. Let $S_{\gamma}$ be the set of all positive and negative rational numbers with $\gamma$ bits before and after the decimal.

- Definition 2 (Random algebraic branching program). Given the parameters $w, d, m$ and $\gamma$, a random $\left(w, d, m, S_{\gamma}\right)$-ABP is a $\left(w, d, m, S_{\gamma}\right)$-ABP with coefficients of the affine forms chosen independently and uniformly at random from $S_{\gamma}{ }^{15}$.

Indeed there is a randomized sampling algorithm which when given the parameters $w, d, m$ and $\gamma$ outputs a random $\left(w, d, m, S_{\gamma}\right)$ - ABP in time $(w, d, m, \gamma)^{O(1)}$. An average-case ABP reconstruction problem can then be posed as follows.

- Problem 3 (Average-case ABP reconstruction). Design an algorithm which when given blackbox access to a polynomial $f$ computed by a random $\left(w, d, m, S_{\gamma}\right)-A B P$, outputs an

\footnotetext{
${ }^{14}$ Our results also hold over finite fields of sufficiently large (meaning, polynomial in the relevant parameters) characteristic.

${ }^{15}$ More generally, $S_{\gamma}$ can be any arbitrarily fixed set containing rational numbers of the form $\frac{p}{q}$, where $p$ and $q$ are $\gamma$ bit integers. For concreteness of the discussion we have fixed $S_{\gamma}$ in a specific way.
} 
ABP computing $f$ with high probability ${ }^{16}$. The desired running time of the algorithm is $(w, d, m, \gamma)^{O(1)}$.

Note that we allow the reconstruction algorithm to output any ABP computing $f$ which may not be a $\left(w, d, m, S_{\gamma}\right)$-ABP. The main requirement is that the running time should be polynomial in $w, d, m$ and $\gamma$.

\subsection{Our result}

We give a solution to the above problem, if the number of variables $m$ and the size of the set $S_{\gamma}$ are greater than $w^{2} d$ and $(m w d)^{2}$ respectively. Observe that if the random affine forms in the matrices $X_{1}$ to $X_{d}$ (as in Definition 2) have more than $w^{2} d$ variables then these affine forms are $\mathbb{F}$-linearly independent with high probability as $S_{\gamma}$ is also sufficiently large. This motivates us to define a full rank ABP. In the following discussion, by homogeneous degree 1 part of an affine form $a_{0}+\sum_{i=1}^{m} a_{i} x_{i}$ we mean $\sum_{i=1}^{m} a_{i} x_{i}$ where $a_{i} \in \mathbb{F}$.

- Definition 4 (Full rank algebraic branching program). A full rank ABP A of width bounded by $w$ and length $d$ is a product expression $X_{1} \cdot X_{2} \ldots X_{d}$, where $X_{1}, X_{2}$ are row and column vectors of lengths $w_{1}$ and $w_{d-1}$ respectively, and for $k \in[2, d-1] X_{k}$ is a $w_{k-1} \times w_{k}$ matrix such that $w_{k} \leq w$ for all $k \in[d-1]$; the entries in $X_{1}$ to $X_{d}$ are affine forms in $\mathbf{x}$ variables and moreover, the homogeneous degree 1 parts of these affine forms are $\mathbb{F}$-linearly independent. We say ABP A has width $\mathbf{w}=\left(w_{1}, w_{2}, \ldots, w_{d-1}\right) \in \mathbb{N}^{d-1}$.

The following is an example of a full rank ABP,

$$
\left[\begin{array}{lll}
1+x_{1}+x_{2} & 2+x_{2}+x_{3} & x_{3}+x_{4}
\end{array}\right]\left[\begin{array}{cc}
1+x_{4}+x_{5} & x_{5}+x_{6} \\
x_{6}+x_{7} & x_{7}+x_{8} \\
x_{8}+x_{9} & 4+x_{9}+x_{10}
\end{array}\right]\left[\begin{array}{c}
3+x_{10}+x_{11} \\
2+x_{11}
\end{array}\right]
$$

A canonical example: Another example of a polynomial computed by a full rank ABP is the iterated matrix multiplication polynomial $\mathrm{IMM}_{\mathbf{w}, d}$, which is the entry of the $1 \times 1$ matrix obtained from a product of $d$ symbolic matrices $X_{1}$ to $X_{d}$ with dimensions as in Definition 4 . The number of variables in $\mathrm{IMM}_{\mathbf{w}, d}$ is $n=w_{1}+\sum_{k=2}^{d-1} w_{k-1} w_{k}+w_{d-1}$. See Definition 2.3 for a slightly detailed definition of $\mathrm{IMM}_{\mathbf{w}, d}$. Generally in the literature, the matrices $X_{1}$ to $X_{d}$ have a uniform dimension $w$ (i.e. $w_{k}=w$ for every $k \in[d-1]$ ) and the polynomial is denoted by $\mathrm{IMM}_{w, d}$. We consider varying dimensions primarily because the algorithm in Theorem 5 below is able to handle this general setting, even if $\mathbf{w}$ is unknown.

Our main result is an efficient randomized algorithm to reconstruct full rank ABP.

- Theorem 5 (Full rank ABP reconstruction). There is a randomized algorithm that takes as input a blackbox for an $m$ variate polynomial $f$ over $\mathbb{F}$ of degree $d \in[5, m]$, and with high probability it does the following: if $f$ is computed by a full rank $A B P$ then the algorithm outputs a full rank ABP computing $f$, else it outputs ' $f$ does not admit a full rank ABP'. The running time is $\operatorname{poly}(m, \beta)^{17}$, where $\beta$ is the bit length of the coefficients of $f$.

\footnotetext{
${ }^{16}$ The probability is taken over the random choice of $f$ (the polynomial computed by a random $\left(w, d, m, S_{\gamma}\right)$ $\mathrm{ABP})$ as well as over the random bits used by the reconstruction algorithm, if it is randomized.

${ }^{17}$ Throughout this article poly $(m)$ denotes a sufficiently large polynomial function in $m$; $\operatorname{poly}(m, \beta)$ is defined similarly.
} 
Remarks: Theorem 5 implies an efficient average-case reconstruction algorithm for ABPs (Problem 3) when $m \geq w^{2} d$ and $\left|S_{\gamma}\right| \geq(m w d)^{2}$, as a random $\left(w, d, m, S_{\gamma}\right)$-ABP is full rank with high probability if $m$ and $\left|S_{\gamma}\right|$ are sufficiently large. The algorithm of Theorem 5 is given in Section 1.5. Following are a couple of remarks on this algorithm:

1. Uniqueness of full rank ABP: Suppose $f$ is computed by a full rank ABP of width $\mathbf{w}=\left(w_{1}, w_{2}, \ldots, w_{d-1}\right)$, and assume ${ }^{18}$ that $w_{k}>1$ for every $k \in[d-1]$. Then the output of the algorithm is a full rank $\mathrm{ABP}$ of width $\mathbf{w}$ or $\left(w_{d-1}, w_{d-2}, \ldots, w_{1}\right)$, with probability at least $1-\frac{1}{\operatorname{poly}(w, d)}$, where $w=\max _{k \in[d-1]}\left\{w_{k}\right\}$. In fact, any full rank ABP computing $f$ is 'unique' up to the symmetries ${ }^{19}$ of iterated matrix multiplication which we study in Section 6.

2. No knowledge of $\mathbf{w}$ : The algorithm does not need a priori knowledge of the width vector $\mathbf{w}$, it only knows the number of variables $m$ and the degree $d$ of $f$. The algorithm is able to derive $\mathbf{w}$ from blackbox access to $f$ (Section 1.5 gives a sketch of how this is done).

Observe that if $f$ is computed by a full rank ABP of width $\mathbf{w}$ then $f$ is an affine projection of the polynomial IMM $\mathbf{I M}_{\mathbf{w}, d}$ via a full rank transformation (see Definition 17). So the above theorem is identical to the theorem below.

- Theorem 6. Given blackbox access to an $m$ variate polynomial $f \in \mathbb{F}[\mathbf{x}]$ of degree $d \in[5, m]$, the problem of checking if there exist $a \mathbf{w} \in \mathbb{N}^{d-1}$, a $B \in \mathbb{F}^{n \times m}$ of rank $n$ equal to the number of variables in $\mathrm{IMM}_{\mathbf{w}, d}$, and $a \mathbf{b} \in \mathbb{F}^{n}$ such that $f=\mathrm{IMM}_{\mathbf{w}, d}(B \mathbf{x}+\mathbf{b})^{20}$, can be solved in randomized $\operatorname{poly}(m, \beta)$ time where $\beta$ is the bit length of the coefficients of $f$. Further, with probability at least $1-\frac{1}{\text { poly }(n)}$, the following is true: the algorithm returns $a \mathbf{w}, a B \in \mathbb{F}^{n \times m}$ of rank $n$, and $a \mathbf{b} \in \mathbb{F}^{n}$ such that $f=\operatorname{IMM}_{\mathbf{w}, d}(B \mathbf{x}+\mathbf{b})$ if such $\mathbf{w}, B$ and $\mathbf{b}$ exist, else it outputs ' $f$ does not admit a full rank $A B P$ '.

A full rank ABP for $f$ can be derived readily, once we compute $\mathbf{w}, B$ and $\mathbf{b}$ as above. Using known results on variable reduction and translation equivalence test (see Section 2.2) proving Theorem 6 reduces in polynomial time to giving an equivalence test (see Definition 18) for the $\mathrm{IMM}_{\mathbf{w}, d}$ polynomial - this reduction is described in Section 1.5.

- Theorem 7 (Equivalence test for IMM). Given blackbox access to a homogeneous $n$ variate polynomial $f \in \mathbb{F}[\mathbf{x}]$ of degree $d \in[5, n]$, where $|\mathbf{x}|=n$, the problem of checking if there exist $a \mathbf{w} \in \mathbb{N}^{d-1}$ and an invertible $A \in \mathbb{F}^{n \times n}$ such that $f=\mathrm{IMM}_{\mathbf{w}, d}(A \mathbf{x})$, can be solved in randomized $\operatorname{poly}(n, \beta)$ time where $\beta$ is the bit length of the coefficients of $f$. Further, with probability at least $1-\frac{1}{\operatorname{poly}(n)}$ the following holds: the algorithm returns $a \mathbf{w}$, and an invertible $A \in \mathbb{F}^{n \times n}$ such that $f=\operatorname{IMM}_{\mathbf{w}, d}(A \mathbf{x})$ if such $\mathbf{w}$ and $A$ exist, else it outputs 'no such w and $A$ exist'.

Remarks: Suppose $f=\mathrm{IMM}_{\mathbf{w}, d}(A \mathbf{x})$ for an invertible $A \in \mathbb{F}^{n \times n}$ and $\mathbf{w}=\left(w_{1}, w_{2}, \ldots, w_{d-1}\right)$. 1. Irreducibility of $\mathrm{IMM}_{\mathbf{w}, d}$ : We can assume without loss of generality that $w_{k}>1$ for every $k \in[d-1]$, implying $\mathrm{IMM}_{\mathbf{w}, d}$ is an irreducible polynomial. If $w_{k}=1$ for some $k \in[d-1]$ then $\mathrm{IMM}_{\mathbf{w}, d}$ is reducible, in which case we use the factorization algorithm in [22] to get blackbox access to the irreducible factors of $f$ and then apply Theorem 7 to each of these irreducible factors (Section 1.5 has more details on this).

\footnotetext{
${ }^{18}$ The first remark after Theorem 7 justifies this assumption.

${ }^{19}$ The stabilizer under the action of the general linear group.

${ }^{20} \mathrm{~A}$ variable set $\mathbf{x}=\left\{x_{1}, \ldots, x_{m}\right\}$ is treated as a column vector $\left(x_{1} \ldots x_{m}\right)^{T}$ in the expression $B \mathbf{x}+\mathbf{b}$ The affine form entries of the column $B \mathbf{x}+\mathbf{b}$ are then plugged in place of the variables of $\mathbf{I M M}_{\mathbf{w}, d}$ (following a variable ordering, like the one mentioned in Section 2.3).
} 
2. Uniqueness of $\mathbf{w}$ and $A$ : Assuming $w_{k}>1$ for every $k \in[d-1]$, it would follow from the proof of the theorem that $\mathbf{w}$ is unique in the following sense: if $f=\operatorname{IMM}_{\mathbf{w}^{\prime}, d}\left(A^{\prime} \mathbf{x}\right)$, where $A^{\prime} \in \mathbb{F}^{n \times n}$ is invertible, then either $\mathbf{w}^{\prime}=\mathbf{w}$ or $\mathbf{w}^{\prime}=\left(w_{d-1}, w_{d-2}, \ldots, w_{1}\right)$. Since $f=X_{1} \cdot X_{2} \ldots X_{d}=X_{d}^{T} \cdot X_{d-1}^{T} \ldots X_{1}^{T}, \mathbf{w}^{\prime}$ can indeed be $\left(w_{d-1}, w_{d-2}, \ldots, w_{1}\right)$. The invertible transformation $A$ is also unique up to the group of symmetries (see Defintion 19) of $\mathrm{IMM}_{\mathbf{w}, d}$ : if $\mathrm{IMM}_{\mathbf{w}, d}(A \mathbf{x})=\mathrm{IMM}_{\mathbf{w}, d}\left(A^{\prime} \mathbf{x}\right)$ then $A A^{\prime-1}$ is in the group of symmetries of $\mathrm{IMM}_{\mathbf{w}, d}$. In Section 6, we determine this group and show that $\mathrm{IMM}_{\mathbf{w}, d}$ is characterized by it.

3. A related result in [16]: Another useful definition of the iterated matrix multiplication polynomial is the trace of a product of $d w \times w$ symbolic matrices - let us denote this polynomial by $\mathrm{IMM}_{w, d}^{\prime}$. Both the variants, $\mathrm{IMM}_{w, d}^{\prime}$ and $\mathrm{IMM}_{w, d}$, are well-studied in the literature and their circuit complexities are polynomially related. However, an equivalence test for one does not immediately give an equivalence test for the other. This is partly because the group of symmetries of $\mathrm{IMM}_{w, d}^{\prime}$ and $\mathrm{IMM}_{w, d}$ are not exactly the same in nature (see Section 6 for a comparison).

Let $\mathbf{x}_{1}, \ldots, \mathbf{x}_{d}$ be the sets of variables in the $d$ matrices of $\mathrm{IMM}_{w, d}^{\prime}$ respectively. A polynomial $f\left(\mathbf{x}_{1}, \ldots, \mathbf{x}_{d}\right)$ is said to be multilinearly equivalent to $\mathrm{IMM}_{w, d}^{\prime}$ if there are invertible $w \times w$ matrices $A_{1}, \ldots, A_{d}$ such that $f=\mathrm{IMM}_{w, d}^{\prime}\left(A_{1} \mathbf{x}_{1}, \ldots, A_{d} \mathbf{x}_{d}\right)$. Grochow [16] showed the following result: Given the knowledge of the variable sets $\mathbf{x}_{1}, \ldots, \mathbf{x}_{d}$, an oracle to find roots of univariate polynomials over $\mathbb{C}$ and blackbox access to a polynomial $f$, there is a randomized algorithm to check whether $f$ is multilinearly equivalent to $\mathrm{IMM}_{w, d}^{\prime}$ using poly $(w, d)$ operations over $\mathbb{C}$. Due to the issue of representing complex numbers, the model of computation for this result may be assumed to be the Blum-ShubSmale model [10]. Theorem 7 is different from the result in [16] in a few ways: First, the equivalence test is for $\mathrm{IMM}_{\mathbf{w}, d}$ instead of $\mathrm{IMM}_{w, d}^{\prime}$. The algorithm in Theorem 7 operates without the knowledge of the variable sets $\mathbf{x}_{1}, \ldots, \mathbf{x}_{d}$ (in fact, without the knowledge of w). It only "sees" $n$ variables $x_{1}, \ldots, x_{n}$ that are input to the blackbox for $f$. Second, there is no requirement of a oracle for finding roots of univariates. The base field is $\mathbb{Q}$ or a field with sufficiently large characteristic and the model of computation is the Turing machine model. Third, Theorem 7 gives a general equivalence test whereas the algorithm in [16] checks only multilinear equivalence.

\subsection{Discussion}

To summarize, our main contribution is a polynomial time randomized equivalence test for $\mathrm{IMM}_{\mathbf{w}, d}$, even if $\mathbf{w}$ is unknown. Although, equivalence testing is an important problem in its own right, Theorem 5 does not address the average-case ABP reconstruction problem quite satisfactorily because of the restriction $m \geq w^{2} d^{21}$. Keeping the conjecture [1] on pseudo-random polynomial family in mind, the more interesting and challenging scenario is when $m \ll w^{2} d$ in Problem 3, and this case remains an open problem. We address this problem partially in an upcoming work (and equivalence tests feature in there too).

A forthcoming work [27]: If the width $w$ of the ABP is a constant, we still need $m=\Omega(d)$, for a random ABP to have full rank and Theorem 5 to be effective. The case of constant width $\mathrm{ABP}$ is interesting in its own right as they capture the complexity of arithmetic

${ }^{21}$ Besides, the model full rank ABP, although natural and powerful, is nevertheless incomplete - not every polynomial $f$ can be computed by a full rank ABP even if $f$ is multilinear (see Observation 60 ). 
formulas. In particular, if a polynomial $g$ is computed by a formula of size $s$ then $g$ can be computed as the $(1,1)$-th entry of a product of $s^{O(1)}$ many $3 \times 3$ matrices with affine form entries [8], and every polynomial computed by a size $s$ width $3 \mathrm{ABP}$ can be computed by a formula of size $s^{O(1)}$. With constant width $\mathrm{ABP}$ in mind, we study a version of Problem 3 (Problem 8 below) in [27], and make progress in certain cases (particularly for $w=3$ ) under the restriction $m \geq w^{2}$; that is for constant width, $m$ only needs to be larger than a constant. Problem 8 is also a natural matrix factorization problem.

- Problem 8 (Average-case matrix factorization). Design an algorithm which when given a $d \in \mathbb{N}$ and blackbox access to $w^{2}$ entries of a matrix $F=X_{1} \cdot X_{2} \ldots X_{d}$, where $X_{1}, X_{2}, \ldots, X_{d}$ are $w \times w$ matrices having entries affine forms in $m$ variables with coefficients chosen independently and uniformly at random from $S_{\gamma}$, computes $d w \times w$ matrices $Y_{1}, Y_{2}, \ldots, Y_{d}$ with affine form entries such that $F=Y_{1} \cdot Y_{2} \ldots Y_{d}$. The desired running time of the algorithm is $\operatorname{poly}(m, w, d, \gamma)$.

As before, we allow the coefficients of the affine forms in $Y_{1}, Y_{2}, \ldots, Y_{d}$ to not belong to $S_{\gamma}$.

In a certain sense, Problem 8 is a relaxed version of Problem 3: We have blackbox access to all the $w^{2}$ polynomials occurring as entries of the matrix product in Problem 8 , whereas in Problem 3 we have blackbox access to just a single polynomial which can be thought of as one entry of a matrix product. Nevertheless, if the coefficients of the affine forms in $X_{1}, X_{2}, \ldots, X_{d}$ are adversarially chosen in Problem 8 (instead of independently and uniformly at random from $S_{\gamma}$ ) then the problem becomes as hard as worst-case formula reconstruction (by [8]), and this makes the above average-case variant interesting to study.

\subsection{Algorithm and proof strategy}

An algorithm for reconstructing full rank ABP is given in Algorithm 1. At first, we trace the steps of this algorithm to show that proving Theorem 6 reduces to proving Theorem 7 using known methods. Then, we give an equivalence test for $\mathrm{IMM}_{\mathbf{w}, d}$ in Algorithm 2, which is the contribution of this work. Some relevant definitions, notations and concepts can be found in Section 2.

\subsubsection{Reduction to equivalence test for IMM}

We are given blackbox access to an $m$ variate polynomial $f(\tilde{\mathbf{x}})$ in Algorithm 1 where $\tilde{\mathbf{x}}=\left\{x_{1}, \ldots, x_{m}\right\}$. Suppose $f=\mathrm{IMM}_{\mathbf{w}^{\prime}, d}\left(B^{\prime} \tilde{\mathbf{x}}+\mathbf{b}^{\prime}\right)$ for some unknown $\mathbf{w}^{\prime} \in \mathbb{N}^{d-1}, \mathbf{b}^{\prime} \in \mathbb{F}^{n}$ and $B^{\prime} \in \mathbb{F}^{n \times m}$ of rank $n$, where $n$ is the number of variables in $\mathrm{IMM}_{\mathbf{w}^{\prime}, d}$.

Variable reduction (Step 2): The number of essential/redundant variables of a polynomial remains unchanged under affine projection via full rank transformation. Since $\mathrm{IMM}_{\mathbf{w}^{\prime}, d}$ has no redundant variables ${ }^{22}$, the number of essential variables of $f$ equals $n$. The algorithm eliminates the $m-n$ redundant variables in $f$ by applying Algorithm 8 and constructs a $C \in \mathrm{GL}(m)$ such that $g=f(C \tilde{\mathbf{x}})$ has only the essential variables $\mathbf{x}=\left\{x_{1}, \ldots, x_{n}\right\}$. It follows that $g=\mathrm{IMM}_{\mathbf{w}^{\prime}, d}\left(A^{\prime} \mathbf{x}+\mathbf{b}^{\prime}\right)$, where $A^{\prime} \in \mathrm{GL}(n)$ is the matrix $B^{\prime} \cdot C$ restricted to the first $n$ columns.

\footnotetext{
${ }^{22}$ Which follows easily from Claim 26.
} 
Equivalence test (Steps 5-9): Since $g=\mathrm{IMM}_{\mathbf{w}^{\prime}, d}\left(A^{\prime} \mathbf{x}+\mathbf{b}^{\prime}\right)$, its $d$-th homogeneous component $g^{[d]}=\mathrm{IMM}_{\mathbf{w}^{\prime}, d}\left(A^{\prime} \mathbf{x}\right)$. In other words, $g^{[d]}$ is equivalent to $\mathrm{IMM}_{\mathbf{w}^{\prime}, d}$ for an unknown $\mathbf{w}^{\prime} \in \mathbb{N}^{d-1}$. At this point, the algorithm calls Algorithm 2 to find a $\mathbf{w}$ and an $A \in \mathrm{GL}(n)$ such that $g^{[d]}=\operatorname{IMM}_{\mathbf{w}, d}(A \mathbf{x})$, and this is achieved with high probability.

Finding a translation (Steps 12-17): As $g=\mathrm{IMM}_{\mathbf{w}^{\prime}, d}\left(A^{\prime} \cdot\left(\mathbf{x}+A^{\prime-1} \mathbf{b}^{\prime}\right)\right)=g^{[d]}\left(\mathbf{x}+A^{-1} \mathbf{b}^{\prime}\right)$, $g$ is translation equivalent to $g^{[d]}$. With high probability, Algorithm 9 finds an $\mathbf{a} \in \mathbb{F}^{n}$ such that $g=g^{[d]}(\mathbf{x}+\mathbf{a})$, implying $g=\mathrm{IMM}_{\mathbf{w}, d}(A \mathbf{x}+A \mathbf{a})$. Thus $\mathbf{b}=A \mathbf{a}$ is a valid translation vector.

Final reconstruction (Steps 20-26): From the previous steps, we have $g=\mathrm{IMM}_{\mathbf{w}, d}(A \mathbf{x}+\mathbf{b})$. Although the variables $\left\{x_{n+1}, \ldots, x_{m}\right\}$ are absent in $g$, if we pretend that $g$ is a polynomial in all the $\tilde{\mathbf{x}}$ variables then $g=\mathrm{IMM}_{\mathbf{w}, d}\left(A_{0} \tilde{\mathbf{x}}+\mathbf{b}\right)$, where $A_{0}$ is an $n \times m$ matrix such that the $n \times n$ submatrix formed by restricting to the first $n$ columns of $A_{0}$ equals $A$ and the remaining $m-n$ columns of $A_{0}$ have all zero entries. Hence $f=g\left(C^{-1} \tilde{\mathbf{x}}\right)=\mathrm{IMM}_{\mathbf{w}, d}\left(A_{0} C^{-1} \tilde{\mathbf{x}}+\mathbf{b}\right)$ which explains the setting $B=A_{0} C^{-1}$ in step 20 . The identity testing in steps $21-23$ takes care of the situation when, to begin with, there are no $\mathbf{w}^{\prime} \in \mathbb{N}^{d-1}, \mathbf{b}^{\prime} \in \mathbb{F}^{n}$ and $B^{\prime} \in \mathbb{F}^{n \times m}$ of rank $n$ such that $f=\mathrm{IMM}_{\mathbf{w}^{\prime}, d}\left(B^{\prime} \tilde{\mathbf{x}}+\mathbf{b}^{\prime}\right)$.

\subsubsection{Equivalence test for IMM}

Algorithm 1 calls Algorithm 2 on a blackbox holding a homogeneous $n$ variate polynomial $f(\mathbf{x})$ of degree $d \leq n$, and expects a $\mathbf{w} \in \mathbb{N}^{d-1}$ and an $A \in \mathrm{GL}(n)$ in return such that $f=\mathrm{IMM}_{\mathbf{w}, d}(A \mathbf{x})$, if such $\mathbf{w}$ and $A$ exist. First, we argue that $f$ can be assumed to be an irreducible polynomial.

Assuming irreducibility of input $\boldsymbol{f}$ in Algorithm 2: The idea is to construct blackbox access to the irreducible factors of $f$ using the efficient randomized polynomial factorization algorithm in [22], and compute full rank ABP for each of these irreducible factors. The ABPs are then connected 'in series' to form a full rank ABP for $f$. This process succeeds with high probability. The details are as follows: If $f$ is not square-free (which can be easily checked using [22]) then $f$ cannot be equivalent to $\mathrm{IMM}_{\mathbf{w}, d}$ for any $\mathbf{w}$, as $\mathrm{IMM}_{\mathbf{w}, d}$ is always square-free. Suppose $f=f_{1} \cdots f_{k}$, where $f_{1}, \ldots, f_{k}$ are distinct irreducible factors of $f$. If there are $\mathbf{w}^{\prime} \in \mathbb{N}^{d-1}$ and $A^{\prime} \in \mathrm{GL}(n)$ such that $f=\mathrm{IMM}_{\mathbf{w}^{\prime}, d}\left(A^{\prime} \mathbf{x}\right.$ ), then the number of essential variables in $f$ is $n$ (as $\mathrm{IMM}_{\mathbf{w}^{\prime}, d}$ has no redundant variables). Also, $f_{1} \cdots f_{k}=h_{1}\left(A^{\prime} \mathbf{x}\right) \cdots h_{k}\left(A^{\prime} \mathbf{x}\right)$ where $h_{1}, \ldots, h_{k}$ are the irreducible factors of $\mathrm{IMM}_{\mathbf{w}^{\prime}, d}$. The irreducible factors of $\mathrm{IMM}_{\mathbf{w}^{\prime}, d}$ are 'smaller IMMs' in disjoint sets of variables ${ }^{23}$. Hence, by uniqueness of factorization, $f_{\ell}$ is computable by a full rank ABP for every $\ell \in[k]$. Let the degree of $f_{\ell}$ be $d_{\ell}$ and $n_{\ell}$ the number of essential variables in $f_{\ell}$. Then $n_{1}+\ldots+n_{k}=n$. Now observe that if we invoke Algorithm 1 on input $f_{\ell}$, it calls Algorithm 2 from within on an irreducible polynomial, as $f_{\ell}$ is homogeneous and irreducible. Algorithm 1 returns a $\mathbf{w}_{\ell} \in \mathbb{N}^{d_{\ell}-1}$ and $B_{\ell} \in \mathbb{F}^{n_{\ell} \times n}$ of rank $n_{\ell}$ such that $f_{\ell}=\mathrm{IMM}_{\mathbf{w}_{\ell}, d_{\ell}}\left(B_{\ell} \mathbf{x}\right)$ (ignoring the translation vector as $f_{\ell}$ is homogeneous). Let $\mathbf{w} \in \mathbb{N}^{d-1}$ be the vector $\left(\mathbf{w}_{1} 1 \mathbf{w}_{2} 1 \ldots 1 \mathbf{w}_{k}\right)^{24}$, and $A \in \mathbb{F}^{n \times n}$ such that the first $n_{1}$ rows of $A$ is $B_{1}$, next $n_{2}$ rows is $B_{2}$, and so on till last $n_{k}$ rows is $B_{k}$. Then, $f=\mathrm{IMM}_{\mathbf{w}, d}(A \mathbf{x})$. Clearly, $A$

\footnotetext{
${ }^{23}$ Recall, $\mathrm{IMM}_{\mathbf{w}, d}$ is irreducible if $w_{k}>1$ for every $k \in[d-1]$ where $\mathbf{w}=\left(w_{1}, \ldots, w_{d-1}\right)$.

${ }^{24}$ The notation means the entries of $\mathbf{w}_{1}$ are followed by 1 , followed by the entries of $\mathbf{w}_{2}$, then a 1 again, and so on.
} 


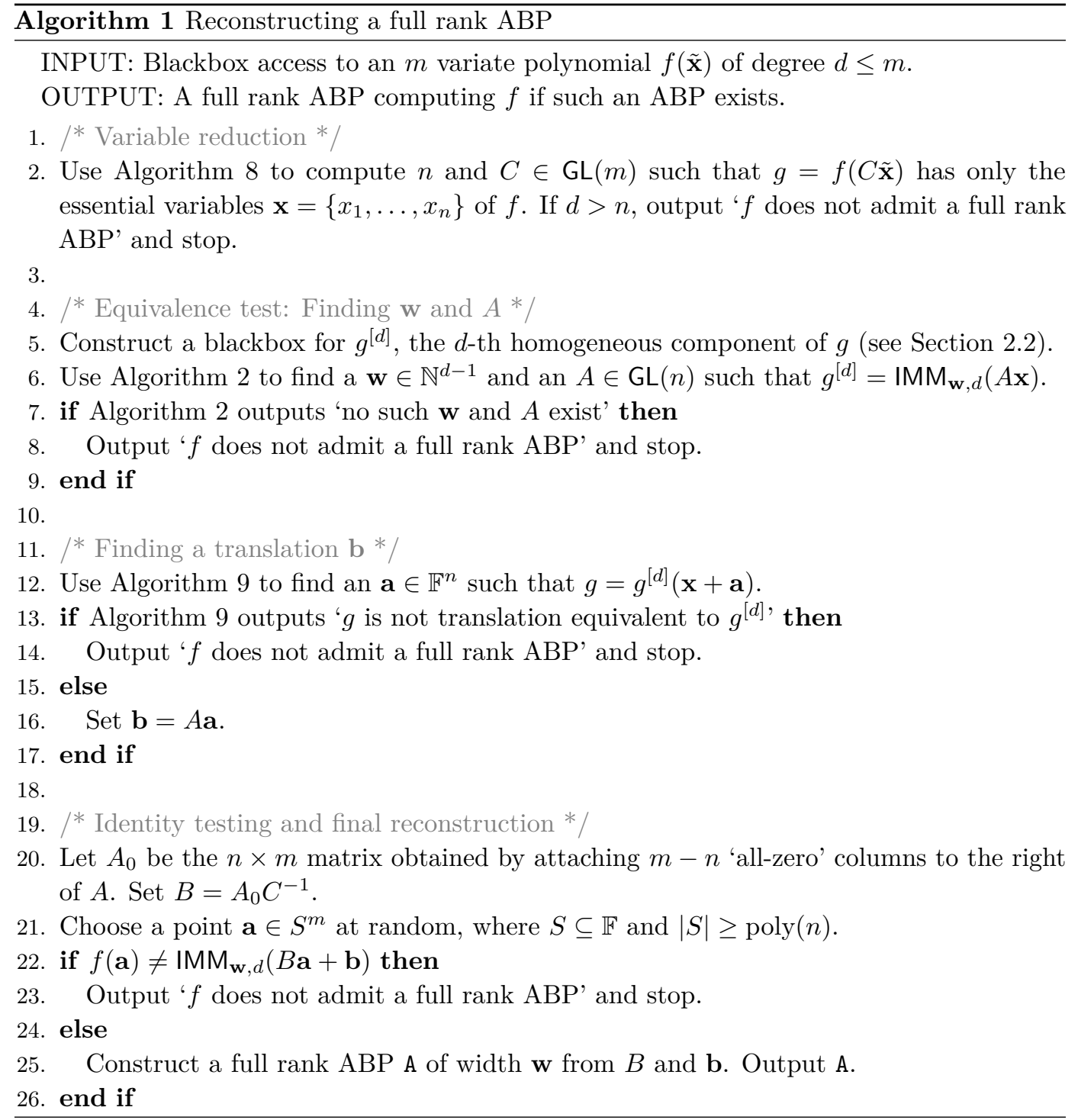

must be in $\mathrm{GL}(n)$ as the number of essential variables of $f$ is $n$. Thus, it is sufficient to describe Algorithm 2 on an input $f$ that is irreducible.

A comparison with [25] and our proof strategy: Kayal [25] gave equivalence tests for the permanent and determinant polynomials by making use of their Lie algebra (see Definition 20). Algorithm 2 also involves Lie algebra of IMM, but there are some crucial differences in the way Lie algebra is used in [25] and in here. The Lie algebra of permanent consists of diagonal matrices and hence commutative. By diagonalizing a basis of $\mathfrak{g}_{f}$ over $\mathbb{C}$, for an $f$ equivalent to permanent, we can reduce the problem to the much simpler permutation and scaling (PS) equivalence problem. The Lie algebra of $n \times n$ determinant, which is isomorphic to $\mathfrak{s l}_{n} \oplus \mathfrak{s l}_{n}$, is not commutative. However, a Cartan subalgebra of $\mathfrak{s l}_{n}$ consists of traceless diagonal matrices. This then helps reduce the problem to PS-equivalence by diagonalizing (over $\mathbb{C}$ ) a basis of the centralizer of a random element in $\mathfrak{g}_{f}$, for an $f$ equivalent to determinant. Both the equivalence tests involve simultaneous diagonalization of matrices over $\mathbb{C}$. It is a bit unclear 
how to carry through this step if the base field is $\mathbb{Q}$ and we insist on low bit complexity. The Lie algebra of IMM is not commutative. Also, we do not know if going to Cartan subalgebra helps, as we would like to avoid the simultaneous diagonalization step. Instead of Cartan subalgebras, we study invariant subspaces (Definition 12) of the Lie algebra $\mathfrak{g}_{\mathrm{IMM}}$. A detailed analysis of the Lie algebra (in Section 3) reveals the structure of the irreducible invariant subspaces of $\mathfrak{g}_{\mathrm{MM}}$. It is observed that these invariant subspaces are intimately connected to the layer spaces (see Definition 15) of any full rank ABP computing $f$. At a conceptual level, this connection helps us reconstruct a full rank ABP. Once we have access to the layer spaces, we can retrieve the unknown width vector $\mathbf{w}$ whence the problem reduces to the easier problem of reconstructing an almost set-multilinear ABP (Definition 29).

We now give some more details on Algorithm 2. Suppose there is a $\mathbf{w} \in \mathbb{N}^{d-1}$ such that $f$ is equivalent to $\mathrm{IMM}_{\mathrm{w}, d}$. The algorithm has four main steps:

1. Computing irreducible invariant subspaces (Steps 2-6): The algorithm starts by computing a basis of the Lie algebra $\mathfrak{g}_{f}$. It then invokes Algorithm 3 to compute bases of the $d$ irreducible invariant subspaces of $\mathfrak{g}_{f}$. Algorithm 3 works by picking a random element $R^{\prime}$ in $\mathfrak{g}_{f}$ and factoring its characteristic polynomial $h=g_{1} \cdots g_{s}$. By computing the closure of vectors (Definition 14) picked from null spaces of $g_{1}\left(R^{\prime}\right), \ldots, g_{s}\left(R^{\prime}\right)$, the algorithm is able to find bases of the required invariant spaces.

2. Computing layer spaces (Step 9): The direct relation between the irreducible invariant spaces of $\mathfrak{g}_{\mathrm{MM}}$ and the layers spaces of any full rank ABP computing $f$ (as shown in Lemma 49) is exploited by Algorithm 5 to compute bases of these layer spaces. This also helps establish that all the layer spaces, except two of them, are 'unique' (see Lemma 48). The second and second-to-last layer spaces of a full rank ABP are not unique; however the bigger space spanned by the first two layer spaces (similarly the last two layer spaces) is unique. Algorithm 5 finds bases for these two bigger spaces along with the $d-2$ remaining layer spaces.

3. Reduction to almost set-multilinear ABP (Steps 12-15): The layer spaces are then correctly reordered in Algorithm 6 using a randomized procedure to compute the appropriate evaluation dimensions (Definition 16). The reordering also yields a valid width vector w. At this point, the problem easily reduces to reconstructing a full rank almost setmultilinear ABP by mapping the bases of the layer spaces to distinct variables. This mapping gives an $\widehat{A} \in \mathrm{GL}(n)$ such that $f(\widehat{A} \mathbf{x})$ is computable by a full rank almost set-multilinear ABP of width w. It is 'almost set-multilinear' (and not 'set-multilinear') as the second and the second-to-last layer spaces are unavailable; instead, two bigger spaces are available as mentioned above.

4. Reconstructing a full rank almost set-mutlilinear ABP (Steps 18-22): Finally, we reconstruct a full rank almost set-mutlilinear ABP computing $f(\widehat{A} \mathbf{x})$ using Algorithm 7. This algorithm is inspired by a similar algorithm for reconstructing set-multilinear ABP in [29], but it is a little different from the latter as we are dealing with an 'almost' set-multilinear

$\mathrm{ABP}$. The reconstructed $\mathrm{ABP}$ readily gives an $A \in \mathrm{GL}(n)$ such that $f=\mathrm{IMM}_{\mathbf{w}, d}(A \mathbf{x})$.

A final identity testing (Steps 25-30) takes care of the situation when, to begin with, there is no $\mathbf{w} \in \mathbb{N}^{d-1}$ that makes $f$ equivalent to $\mathrm{IMM}_{\mathbf{w}, d}$.

\section{Preliminaries}

\subsection{Notations and definitions}

The group of invertible $n \times n$ matrices over $\mathbb{F}$ is represented by $\mathrm{GL}(n, \mathbb{F})$. Since $\mathbb{F}$ is fixed to be the field of rationals, we omit $\mathbb{F}$ and write $\mathrm{GL}(n)$. Natural numbers are denoted by 


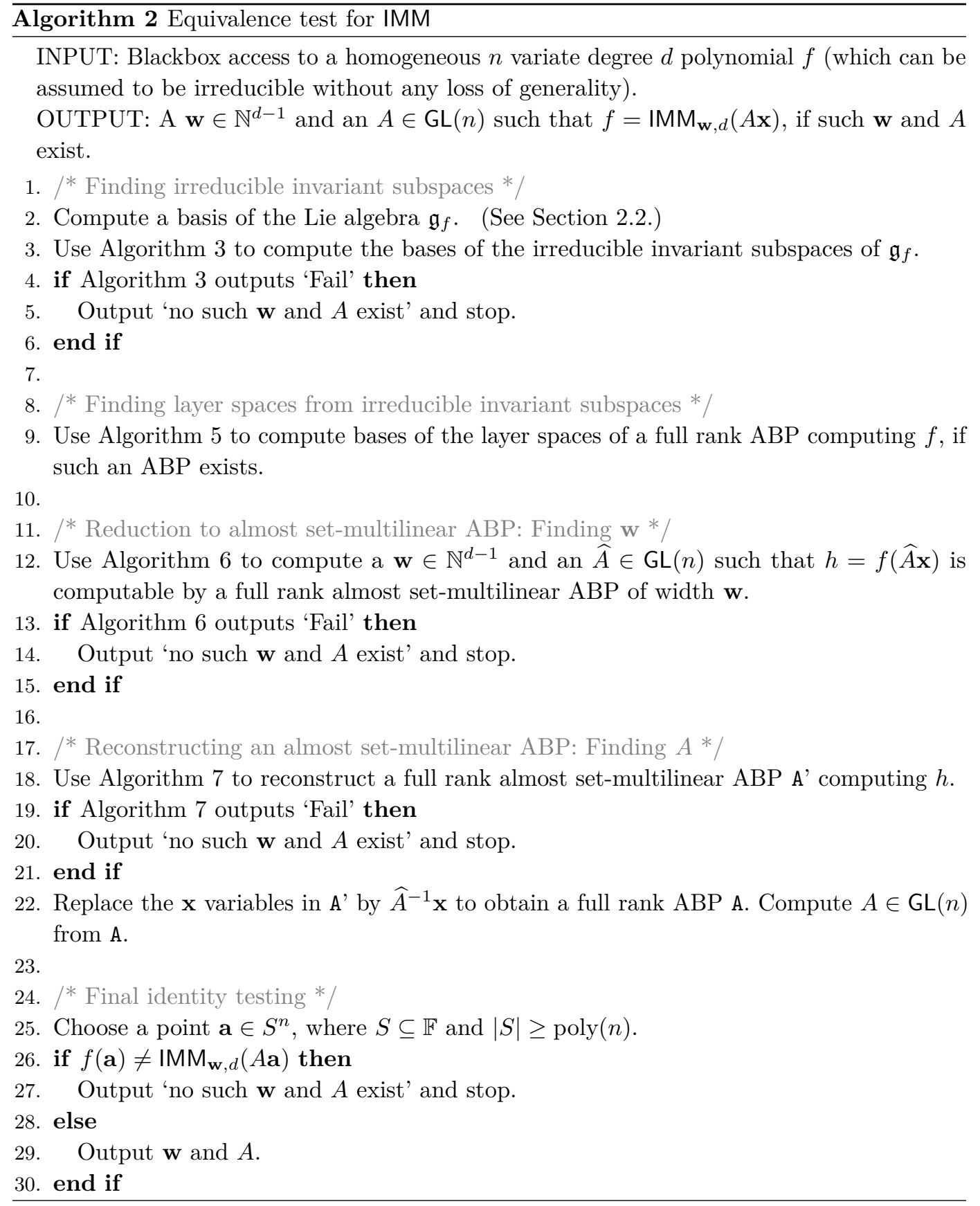

$\mathbb{N}=\{1,2, \ldots\}$. As a convention, we use $\mathbf{x}, \mathbf{y}$ and $\mathbf{z}$ to denote sets of variables, capital letters $A, B, C$ and so on to denote matrices, calligraphic letters like $\mathcal{U}, \mathcal{V}, \mathcal{W}$ to denote vector spaces over $\mathbb{F}$, and bold small letters like $\mathbf{u}, \mathbf{v}, \mathbf{w}$ to denote vectors in these spaces. All vectors considered in this article are column vectors, unless mentioned otherwise. An affine form in $\mathbf{x}=\left\{x_{1}, x_{2}, \ldots, x_{n}\right\}$ variables is $a_{0}+\sum_{i=1}^{n} a_{i} x_{i}$ where for $i \in[0, d] a_{i} \in \mathbb{F}$, and if $a_{0}=0$ then we call it a linear form. The first order partial derivative of the polynomial $f(\mathbf{x})$ with respect to $x_{i}$ is denoted as $\partial_{x_{i}}(f(\mathbf{x}))$. Below we set up some notations and terminologies. 


\subsubsection{Linear Algebra}

- Definition 9 (Direct sum). Let $\mathcal{U}, \mathcal{W}$ be subspaces of a vector space $\mathcal{V}$. Then $\mathcal{V}$ is said to be the direct sum of $\mathcal{U}$ and $\mathcal{W}$ denoted $\mathcal{V}=\mathcal{U} \oplus \mathcal{W}$, if $V=\mathcal{U}+\mathcal{W}$ and $\mathcal{U} \cap \mathcal{W}=\{\mathbf{0}\}$.

For $\mathcal{U}, \mathcal{W}$ subspaces of a vector space $\mathcal{V}, \mathcal{V}=\mathcal{U} \oplus \mathcal{W}$ if and only if for every $\mathbf{v} \in \mathcal{V}$ there exist unique $\mathbf{u} \in \mathcal{U}$ and $\mathbf{w} \in \mathcal{W}$ such that $\mathbf{v}=\mathbf{u}+\mathbf{w}$. Hence, $\operatorname{dim}(\mathcal{V})=\operatorname{dim}(\mathcal{U})+\operatorname{dim}(\mathcal{W})$.

- Definition 10 (Null space). Null space $\mathcal{N}$ of a matrix $M \in \mathbb{F}^{n \times n}$ is the space of all vectors $\mathbf{v} \in \mathbb{F}^{n}$, such that $M \mathbf{v}=\mathbf{0}$.

- Definition 11 (Coordinate subspace). Let $e_{i}=(0, \ldots, 1, \ldots, 0)$ be the unit vector in $\mathbb{F}^{n}$ with 1 at the $i$-th position and all other coordinates zero. A coordinate subspace of $\mathbb{F}^{n}$ is a space spanned by a subset of the $n$ unit vectors $\left\{e_{1}, e_{2}, \ldots, e_{n}\right\}$.

- Definition 12 (Invariant subspace). Let $M_{1}, M_{2}, \ldots, M_{k} \in \mathbb{F}^{n \times n}$. A subspace $\mathcal{U} \subseteq \mathbb{F}^{n}$ is called an invariant subspace of $\left\{M_{1}, M_{2}, \ldots, M_{k}\right\}$ if $M_{i} \mathcal{U} \subseteq \mathcal{U}$ for every $i \in[k]$. A nonzero invariant subspace $\mathcal{U}$ is irreducible if there are no invariant subspaces $\mathcal{U}_{1}$ and $\mathcal{U}_{2}$ such that $\mathcal{U}=\mathcal{U}_{1} \oplus \mathcal{U}_{2}$, where $\mathcal{U}_{1}$ and $\mathcal{U}_{2}$ are properly contained in $\mathcal{U}$.

The following observation is immediate.

- Observation 13. If $\mathcal{U}$ is an invariant subspace of $\left\{M_{1}, M_{2}, \ldots, M_{k}\right\}$ then for every $M \in$ $\mathcal{L} \stackrel{\text { def }}{=} \operatorname{span}_{\mathbb{F}}\left\{M_{1}, M_{2}, \ldots, M_{k}\right\}, M \mathcal{U} \subseteq \mathcal{U}$. Hence we say $\mathcal{U}$ is an invariant subspace of $\mathcal{L}, a$ space generated by matrices.

- Definition 14 (Closure of a vector). The closure of a vector $\mathbf{v} \in \mathbb{F}^{n}$ under the action of a space $\mathcal{L}$ spanned by a set of $n \times n$ matrices is the smallest invariant subspace of $\mathcal{L}$ containing $\mathbf{v}$.

Here, 'smallest' is with regard to dimension of invariant subspaces. Since intersection of two invariant subspaces is also an invariant subspace of $\mathcal{L}$, the smallest invariant subspace of $\mathcal{L}$ containing $\mathbf{v}$ is unique and is contained in every invariant subspace of $\mathcal{L}$ containing $\mathbf{v}$. Algorithm 4 in Section 4.2 computes the closure of a given vector $\mathbf{v}$ under the action of $\mathcal{L}$ whose basis is given.

By identifying a linear form $\sum_{i=1}^{n} a_{i} x_{i}$ with the vector $\left(a_{1}, \ldots, a_{n}\right) \in \mathbb{F}^{n}$ (and vice versa), we can associate the following vector spaces with an ABP.

- Definition 15 (Layer spaces of an ABP). Let $X_{1} \cdot X_{2} \ldots X_{d}$ be a full rank ABP A of length $d$ and width $\mathbf{w}=\left(w_{1}, w_{2}, \ldots, w_{d-1}\right)$, where $X_{1}$ to $X_{d}$ are as in Definition 4 . Let $\mathcal{X}_{i}$ be the vector space in $\mathbb{F}^{n}$ spanned by the homogeneous degree 1 parts of the affine forms ${ }^{25}$ in $X_{i}$ for $i \in[d]$; the spaces $\mathcal{X}_{1}, \mathcal{X}_{2}, \ldots, \mathcal{X}_{d}$ are called the layer spaces of $\mathrm{A}$.

\subsubsection{Evaluation dimension}

The rank of the partial derivative matrix of a polynomial $f$ was introduced in [34] and used subsequently in several works on lower bound, polynomial identity testing and circuit reconstruction (see [39]). The following definition (which makes the notion well defined for fields of finite characteristic) appears in $[14]^{26}$.

\footnotetext{
${ }^{25}$ Identify linear forms with vectors in $\mathbb{F}^{n}$ as mentioned above.

${ }^{26}$ They attributed the definition to Ramprasad Saptharishi.
} 
- Definition 16 (Evaluation dimension). The evaluation dimension of a polynomial $g \in \mathbb{F}[\mathbf{x}]$ with respect to a set $\mathbf{x}^{\prime} \subseteq \mathbf{x}$, denoted as $\operatorname{Evaldim}_{\mathbf{x}^{\prime}}(g)$, is defined as

$$
\operatorname{dim}\left(\operatorname{span}_{\mathbb{F}}\left\{\left.g(\mathbf{x})\right|_{\forall x_{j} \in \mathbf{x}^{\prime} x_{j}=\alpha_{j}}: \alpha_{j} \in \mathbb{F} \text { for every } x_{j} \in \mathbf{x}^{\prime}\right\}\right) .
$$

\subsubsection{Affine projection and equivalence testing}

Studying polynomials by applying linear transformations (from suitable matrix groups) on the variables is at the heart of invariant theory.

Definition 17 (Affine projection). An $m$ variate polynomial $f$ is an affine projection of a $n$ variate polynomial $g$, if there exists a matrix $A \in \mathbb{F}^{n \times m}$ and a $\mathbf{b} \in \mathbb{F}^{n}$ such that $f(\mathbf{x})=g(A \mathbf{x}+\mathbf{b})$.

In [25], it was shown that given an $m$ variate polynomial $f$ and an $n$ variate polynomial $g$, checking whether $f$ is an affine projection of $g$ is NP-hard, even if $f$ and $g$ are given in the dense representation (that is as list of coefficients of the monomials). In the above definition, we say $f$ is an affine projection of $g$ via a full rank transformation, if $m \geq n$ and $A$ has rank $n$. In the affine projection via full rank transformation problem, we are given an $m$ variate polynomial $f$ and an $n$ variate polynomial $g$ in some suitable representation, and we need to determine if $f$ is an affine projection of $g$ via a full rank transformation. $[24,25]$ studied the affine projection via full rank transformation problem for $g$ coming from fixed families and gave polynomial time randomized algorithms to check whether a degree $d$ polynomial $f$ given as blackbox is an affine projection of $g$ via a full rank transformation, where $g$ is the elementary symmetric polynomial/permanent/determinant/power symmetric polynomial or sum-of-products polynomial. As observed in [25], variable reduction and translation equivalence test (described in Section 2.2) help reduce the affine projection via full rank transformation problem to equivalence testing (see also Section 1.5).

- Definition 18 (Equivalent polynomials). An $n$ variate polynomial $f$ is equivalent to an $n$ variate polynomial $g$, if there exists a matrix $A \in \mathrm{GL}(n)$ such that $f(\mathbf{x})=g(A \mathbf{x})$.

The equivalence testing problem asks us to check if two $n$ variate polynomials $f$ and $g$ (given in some suitable representation) are equivalent. This problem is at least as hard as the graph isomorphism problem even when $f$ and $g$ are cubic forms given in dense representation [4]. There is a cryptographic application [35] that assumes the problem is hard also in the average-case for bounded degree $f$ and $g$ given in dense representation. If we restrict to checking if $f$ and $g$ are equivalent via a permutation matrix $A$, then the problem is shown to be in NP $\cap$ coAM [41].

\subsubsection{Group of symmetries and Lie algebra}

- Definition 19 (Group of symmetries). The group of symmetries of a polynomial $g \in \mathbb{F}[\mathbf{x}]$ in $n$ variables, denoted as $\mathcal{G}_{g}$, is the set of all $A \in \mathrm{GL}(n)$ such that $g(A \mathbf{x})=g(\mathbf{x})$.

The proof of Theorem 7 involves an analysis of the Lie algebra of the group of symmetries of $\mathrm{IMM}_{\mathbf{w}, d}$. We will abuse terminology slightly and say the Lie algebra of a polynomial to mean the Lie algebra of the group of symmetries of the polynomial. We will work with the following definition of Lie algebra of a polynomial (see [25]).

- Definition 20 (Lie algebra of a polynomial). The Lie algebra of a polynomial $f \in$ $\mathbb{F}\left[x_{1}, x_{2}, \ldots, x_{n}\right]$ denoted as $\mathfrak{g}_{f}$ is the set of all $n \times n$ matrices $E=\left(e_{i j}\right)_{i, j \in[n]}$ in $\mathbb{F}^{n \times n}$ such that $\sum_{i, j \in[n]} e_{i j} x_{j} \cdot \frac{\partial f}{\partial x_{i}}=0$. 
Remark: Observe that $\mathfrak{g}_{f}$ is a subspace of $\mathbb{F}^{n \times n}$. It can also be shown that the space $\mathfrak{g}_{f}$ satisfies the Lie bracket property: For any $E_{1}, E_{2} \in \mathfrak{g}_{f},\left[E_{1}, E_{2}\right] \stackrel{\text { def }}{=} E_{1} E_{2}-E_{2} E_{1}$ is also in $\mathfrak{g}_{f}$. We would not be needing this property, but would just use the vector space feature of $\mathfrak{g}_{f}$. The proof of the following well known fact is given in [25], see also Section 7.1 for a proof.

- Claim 21. If $f(\mathbf{x})=g(A \mathbf{x})$, where $f$ and $g$ are both $n$ variate polynomials and $A \in \mathrm{GL}(n)$, then the Lie algebra of $f$ is a conjugate of the Lie algebra of $g$ via $A$, i.e. $\mathfrak{g}_{f}=\left\{A^{-1} E A: E \in\right.$ $\left.\mathfrak{g}_{g}\right\}=: A^{-1} \mathfrak{g}_{g} A$.

The following observation relates the invariant subspaces of the Lie algebras of two equivalent polynomials.

- Observation 22. Suppose $f(\mathbf{x})=g(A \mathbf{x})$, where $\mathbf{x}=\left\{x_{1}, x_{2}, \ldots, x_{n}\right\}$ and $A \in \mathrm{GL}(n)$. Then $\mathcal{U} \in \mathbb{F}^{n}$ is an invariant subspace of $\mathfrak{g}_{g}$ if and only if $A^{-1} \mathcal{U}$ is an invariant subspace of $\mathfrak{g}_{f}$.

Proof. $\mathcal{U}$ is an invariant subspace of $\mathfrak{g}_{g}$ implies, for all $E \in \mathfrak{g}_{g}, E \mathcal{U} \subseteq \mathcal{U}$. Consider $E^{\prime} \in \mathfrak{g}_{f}$, using Claim 21 we know there exists $E \in \mathfrak{g}_{g}$ such that $A E^{\prime} A^{-1}=E$. Since $\mathcal{U}$ is an invariant subspace of $A E^{\prime} A^{-1}, A^{-1} \mathcal{U}$ is an invariant subspace of $E^{\prime}$. The proof of the other direction is similar.

\subsection{Algorithmic preliminaries}

We record some of the basic algorithmic tasks on polynomials that can be performed efficiently and which we require at different places in our algorithms and proofs.

\subsubsection{Computing homogeneous components of $f$}

The $i$-th homogeneous component (or the homogeneous degree $i$ part) of a degree $d$ polynomial $f$, denoted as $f^{[i]}$ is the sum of the degree $i$ monomials with coefficients as in $f$. Clearly, $f=f^{[d]}+f^{[d-1]}+\cdots+f^{[0]}$. Given an $n$ variate degree $d$ polynomial $f$ as a blackbox, there is an efficient algorithm to compute blackboxes for the $d$ homogeneous components of $f$. The idea is to multiply each variable by a new formal variable $t$, and then interpolate the coefficients of $t^{0}, t^{1}, \ldots, t^{d}$; the coefficient of $t^{i}$ is $f^{[i]}$.

\subsubsection{Computing derivatives of $f$}

Given a polynomial $f\left(x_{1}, x_{2}, \ldots, x_{n}\right)$ of degree $d$ as a blackbox, we can efficiently construct blackboxes for the derivatives $\partial_{x_{i}} f$, for all $i \in[n]$. The following observation suggests that it is sufficient to construct blackboxes for certain homogeneous components.

- Observation 23. If $g\left(x_{1}, x_{2}, \ldots, x_{n}\right)$ is a homogeneous polynomial of degree $d$ then for all $i \in[n] \partial_{x_{i}} g=\sum_{j=1}^{d} j \cdot x_{i}^{j-1}\left[g\left(x_{1}, x_{2}, \ldots, x_{i-1}, 1, x_{i+1}, \ldots, x_{n}\right)\right]^{[d-j]}$.

For every $i \in[n]$, constructing a blackbox for $\partial_{x_{i}} f$ is immediate from the above observation as $\partial_{x_{i}} f=\partial_{x_{i}} f^{[d]}+\partial_{x_{i}} f^{[d-1]}+\cdots+\partial_{x_{i}} f^{[1]}$.

\subsubsection{Space of linear dependencies of polynomials}

Let $f_{1}, f_{2}, \ldots, f_{m}$ be $n$ variate polynomials in $\mathbb{F}[\mathbf{x}]$ with degree bounded by $d$. The set $\mathcal{U}=\left\{\left(a_{1} a_{2} \ldots a_{m}\right)^{T} \in \mathbb{F}^{m} \mid \sum_{j \in[m]} a_{j} f_{j}=0\right\}$, called the space of $\mathbb{F}$-linear dependencies of $f_{1}, f_{2}, \ldots, f_{m}$ is a subspace of $\mathbb{F}^{m}$. We would like to find a basis of the space $\mathcal{U}$ given 
blackbox access to $f_{1}, f_{2}, \ldots, f_{m}$. Suppose the dimension of the $\mathbb{F}$-linear space spanned by the polynomials $f_{1}, f_{2}, \ldots, f_{m}$ is $m-r$ then $\operatorname{dim}(\mathcal{U})=r$. An algorithm to find a basis of $\mathcal{U}$ can be derived from the following claim.

- Claim 24. With probability at least $1-\frac{1}{\text { poly }(n)}$, the rank of the matrix $M=\left(f_{j}\left(\mathbf{b}_{i}\right)\right)_{i, j \in[m]}$ is $m-r$ where $\mathbf{b}_{1}, \mathbf{b}_{2}, \ldots, \mathbf{b}_{m}$ are chosen independently and uniformly at random from $S^{n} \subset \mathbb{F}^{n}$ with $|S|=d m \cdot \operatorname{poly}(n)$.

The proof of the claim which involves an application of the Schwartz-Zippel lemma is given in Section 7.1. The space $\mathcal{U}$ equals the null space of $M$ with high probability.

\subsubsection{Eliminating redundant variables}

- Definition 25 (Essential and redundant variables). We say an $n$ variate polynomial $f$ has $s$ essential variables if there exists an $A \in \mathrm{GL}(n)$ such that $f(A \mathbf{x})$ is an $s$ variate polynomial and there exists no $A^{\prime} \in \mathrm{GL}(n)$ such that $f\left(A^{\prime} \mathbf{x}\right)$ is a $t$ variate polynomial where $t<s$. An $n$ variate polynomial has $r$ redundant variables if it has $s=n-r$ essential variables.

If the number of essential variables in a polynomial $f\left(x_{1}, x_{2}, \ldots, x_{n}\right)$ is $s$ then without loss of generality we can assume that the first $s$ variables $x_{1}, x_{2}, \ldots, x_{s}$ are essential variables and the remaining variables are redundant. An algorithm to eliminate the redundant variables of a polynomial was considered in [12], and it was shown that if the coefficients of a polynomial are given as input then we can eliminate the redundant variables in polynomial time. Further, [24] gave an efficient randomized algorithm to eliminate the redundant variables in a polynomial given as blackbox. For completeness, we give the algorithm in [24] as part of the following claim.

- Claim 26. Let $r$ be the number of redundant variables in an $n$ variate polynomial $f$ of degree $d$. Then the dimension of the space $\mathcal{U}$ of $\mathbb{F}$-linear dependencies of $\left\{\partial_{x_{i}} f \mid i \in[n]\right\}$ is $r$. Moreover, we can construct an $A \in \mathrm{GL}(n)$ in randomized $\operatorname{poly}(n, d, \beta)$ time such that $f(A \mathbf{x})$ is free of the set of variables $\left\{x_{n-r+1}, x_{n-r+2}, \ldots, x_{n}\right\}$, where $\beta$ is the bit length of the coefficients of $f$.

The proof is given in Section 7.1.

\subsubsection{Efficient translation equivalence test}

Two $n$ variate degree $d$ polynomials $f, g \in \mathbb{F}[\mathbf{x}]$ are translation equivalent (also called shift equivalent in [13]) if there exists a point $\mathbf{a} \in \mathbb{F}^{n}$ such that $f(\mathbf{x}+\mathbf{a})=g(\mathbf{x})$. Translation equivalence test takes input blackbox access to two $n$ variate polynomials $f$ and $g$, and outputs an $\mathbf{a} \in \mathbb{F}^{n}$ such that $f(\mathbf{x}+\mathbf{a})=g(\mathbf{x})$ if $f$ and $g$ are translation equivalent else outputs ' $f$ and $g$ are not translation equivalent'. As before, let $\beta$ be the bit lengths of the coefficients of $f$ and $g$. A randomized poly $(n, d, \beta)$ time algorithm is presented in [13] to test translation equivalence and find an $\mathbf{a} \in \mathbb{F}^{n}$ such that $f(\mathbf{x}+\mathbf{a})=g(\mathbf{x})$, if such an a exists. Another randomized test was mentioned in [25], which we present as proof of the following lemma in Section 7.1.

Lemma 27. There is a randomized algorithm that takes input blackbox access to two $n$ variate, degree $d$ polynomials $f$ and $g$, and with probability at least $1-\frac{1}{\text { poly }(n)}$ does the following: if $f$ is translation equivalent to $g$, outputs an $\mathbf{a} \in \mathbb{F}^{n}$ such that $f(\mathbf{x}+\mathbf{a})=g(\mathbf{x})$, else outputs ' $f$ and $g$ are not translation equivalent'. The running time of the algorithm is $\operatorname{poly}(n, d, \beta)$, where $\beta$ is the bit length of the coefficients of $f$ and $g$. 


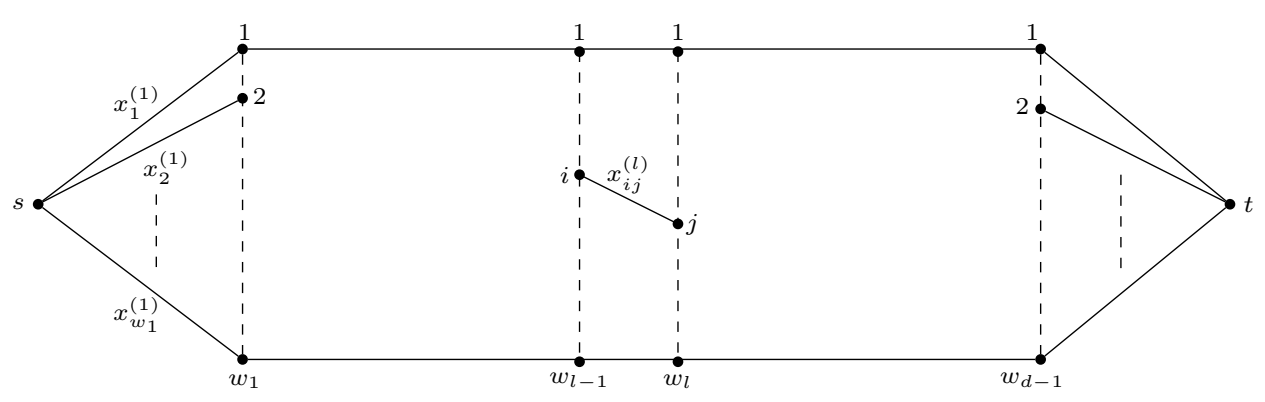

Figure 1 Naming of variables in $\mathrm{IMM}_{\mathbf{w}, d}$.

\subsubsection{Computing basis of Lie algebra}

The proof of the following lemma is given in [25], for completeness we include a proof in Section 7.1.

- Lemma 28. There is a randomized algorithm which when given blackbox access to an $n$ variate degree d polynomial $f$, computes a basis of $\mathfrak{g}_{f}$ with probability at least $1-\frac{1}{\operatorname{poly}(n)}$ in time $\operatorname{poly}(n, d, \beta)$ where $\beta$ is the bit length of the coefficients in $f$.

\subsection{Iterated matrix multiplication polynomial}

Let $\mathbf{w}=\left(w_{1}, w_{2}, \ldots, w_{d-1}\right) \subseteq \mathbb{N}^{d-1}$. Suppose $Q_{1}=\left(x_{1}^{(1)} x_{2}^{(1)} \ldots x_{w_{1}}^{(1)}\right), Q_{d}^{T}=\left(x_{1}^{(d)} x_{2}^{(d)} \ldots\right.$ $\left.x_{w_{d-1}}^{(d)}\right)$ be row vectors, and for $k \in[2, d-1], Q_{k}=\left(x_{i j}^{(k)}\right)_{i \in\left[w_{k-1}\right], j \in\left[w_{k}\right]}$ be a $w_{k-1} \times w_{k}$ matrix, where for $i \in\left[w_{1}\right] x_{i}^{(1)}$, for $i \in\left[w_{d-1}\right] x_{i}^{(d)}$ and for $i \in\left[w_{k-1}\right], j \in\left[w_{k}\right] x_{i j}^{(k)}$ are distinct variables. The iterated matrix multiplication polynomial $\mathrm{IMM}_{\mathbf{w}, d}$ is the entry of the $1 \times 1$ matrix obtained from the product $\prod_{i=1}^{d} Q_{i}$. When $d$ and $\mathbf{w}$ are clear from the context, we drop the subscripts and simply represent it by IMM. For all $k \in[d]$, we denote the set of variables in $Q_{k}$ as $\mathbf{x}_{k}$; Figure 1 depicts an $\mathrm{ABP}$ computing $\mathrm{IMM}_{\mathbf{w}, d}$ when the width is uniform, that is $w_{1}=w_{2}=\cdots=w_{d-1}$.

Ordering of variables in $\mathbf{I} \mathbf{M M}_{\mathbf{w}, \boldsymbol{d}}$ : $\quad$ From here on we will assume that the variables $\mathbf{x}_{1} \uplus \mathbf{x}_{2} \uplus$ $\cdots \uplus \mathbf{x}_{d}$ are ordered as follows: For $i<j$, the $\mathbf{x}_{i}$ variables have precedence over the $\mathbf{x}_{j}$ variables. Among the $\mathbf{x}_{l}$ variables, we follow column-major ordering, i.e $x_{11}^{(l)} \succ \cdots \succ x_{w_{l-1} 1}^{(l)} \succ \cdots \succ$ $x_{1 w_{l}}^{(l)} \succ \cdots \succ x_{w_{l-1} w_{l}}^{(l)}$. We would also refer to the variables of IMM as $\mathbf{x}=\left\{x_{1}, x_{2}, \ldots, x_{n}\right\}$ where $x_{i}$ is the $i$-th variable according to this ordering ${ }^{27}$, and $n=w_{1}+\sum_{k=2}^{d-1} w_{k-1} w_{k}+w_{d-1}$ is the total number of variables in IMM. For $A \in \mathbb{F}^{n \times n}$ we can naturally index the rows and columns of $A$ by the $\mathbf{x}$ variables such that the $i$-th row or column is indexed by the $i$-th variable.

\subsection{Almost set-multilinear ABP and a canonical representation}

In the proof of Theorem 7 , we eventually reduce the equivalence test problem to checking whether there exists an $A \in \mathrm{GL}(n)$, such that an input polynomial $h(\mathbf{x})$ (given as blackbox)

${ }^{27}$ The justification for identifying the variables $\mathbf{x}$ of $f$ with the variables of $\mathrm{IMM}_{\mathbf{w}, d}$ in this order is as follows: If $f$ is equivalent to $\mathrm{IMM}_{\mathbf{w}, d}$ then $f$ is also equivalent to $\mathrm{IMM}_{\mathbf{w}, d}(\mathbf{x})$ whose variables $\left\{x_{1}, \ldots, x_{n}\right\}$ are ordered as above. That $\mathbf{w}$ is a priori unknown to Algorithm 2 does not matter here. 
equals $\mathrm{IMM}_{\mathbf{w}, d}(A \mathbf{x})$, where $\mathbf{w}$ is known, $\mathbf{x}$ is the variables of $\mathrm{IMM}_{\mathbf{w}, d}$, and $A$ satisfies the following properties:

1. For all $k \in[d] \backslash\{2, d-1\}$, the rows indexed by $\mathbf{x}_{k}$ variables contain zero entries in columns indexed by variables other than $\mathbf{x}_{i}$.

2. The rows indexed by $\mathbf{x}_{2}$ and $\mathbf{x}_{d-1}$ variables contain zero entries in columns indexed by variables other than $\mathbf{x}_{1} \uplus \mathbf{x}_{2}$ and $\mathbf{x}_{d-1} \uplus \mathbf{x}_{d}$ respectively.

If there exists such a block-diagonal matrix $A$ then we say $h$ is computed by a full rank almost set-multilinear ABP as defined below.

- Definition 29 (Full rank almost set-multilinear ABP). A full rank almost set-multilinear $\mathrm{ABP}$ of width $\mathbf{w}=\left(w_{1}, w_{2}, \ldots, w_{d-1}\right)$ and length $d$ is a product of $d$ matrices, $X_{1} \cdot X_{2} \ldots X_{d}$, where $X_{k}$ 's are as in Definition 4 but with linear forms as entries. The linear forms in $X_{k}$ are in $\mathbf{x}_{k}$ variables, for all $k \in[d] \backslash\{2, d-1\}$, and for $X_{2}$ and $X_{d-1}$ the linear forms are in $\mathbf{x}_{1} \uplus \mathbf{x}_{2}$ and $\mathbf{x}_{d-1} \uplus \mathbf{x}_{d}$ variables respectively, where $\mathbf{x}_{1} \uplus \mathbf{x}_{2} \cdots \uplus \mathbf{x}_{d}$ is the set of variables in $\mathrm{IMM}_{\mathbf{w}, d}$.

Conventionally, in the definition of set-multilinear ABP, the entries of $X_{i}$ are linear forms in just $\mathbf{x}_{i}$ variables - the ABP in the above definition is almost set-multilinear as matrices $X_{2}$ and $X_{d-1}$ violate this condition. An efficient randomized reconstruction algorithm for set-multilinear ABP follows from [29]. In order to apply a similar reconstruction algorithm to full rank almost set-multilinear ABPs, we fix a canonical representation for the first two and the last two matrices as explained below.

Canonical form or representation: We say a full rank almost set-multilinear ABP of width $\mathbf{w}$ is in canonical form if the following hold:

(1a) $X_{1}=\left(x_{1}^{(1)} x_{2}^{(1)} \ldots x_{w_{1}}^{(1)}\right)$,

(1b) the linear forms in $X_{2}$ are such that for $l, i \in\left[w_{1}\right]$ and $l<i$, the variable $x_{l}^{(1)}$ has a zero coefficient in the $(i, j)$-th entry (linear form) of $X_{2}$, where $j \in\left[w_{2}\right]$.

(2a) $X_{d}=\left(x_{1}^{(d)} x_{2}^{(d)} \ldots x_{w_{d-1}}^{(d)}\right)^{T}$,

(2b) the linear forms in $X_{d-1}$ are such that for $l, j \in\left[w_{d-1}\right]$ and $l<j$, the variable $x_{l}^{(d)}$ has

a zero coefficient in the $(i, j)$-th entry (linear form) of $X_{d-1}$, where $i \in\left[w_{d-2}\right]$.

The following claim states that for every full rank almost set-multilinear ABP there is another ABP in canonical form computing the same polynomial, and the latter can be computed efficiently.

- Claim 30. Let $h$ be an $n$ variate, degree d polynomial computable by a full rank almost set-multilinear $A B P$ of width $\mathbf{w}=\left(w_{1}, w_{2}, \ldots, w_{d-1}\right)$ and length $d$. There is a randomized algorithm that takes input blackbox access to $h$ and the width vector $\mathbf{w}$, and outputs a full rank almost set-multilinear ABP of width $\mathbf{w}$ in canonical form computing $h$, with probability at least $1-\frac{1}{\operatorname{poly}(n)}$. The running time of the algorithm is $\operatorname{poly}(n, \beta)$, where $\beta$ is the bit length of the coefficients of $h$.

We prove the claim in Section 5.3. The algorithm is similar to reconstruction of set-multilinear ABP in [29], except that the latter needs to be adapted suitably as we are dealing with almost set-multilinear ABP.

\section{Lie algebra of IMM}

Dropping the subscripts $\mathbf{w}$ and $d$, we refer to $\mathrm{IMM}_{\mathbf{w}, d}$ as IMM. We show that the Lie algebra, $\mathfrak{g}_{\text {IMM }}$ consists of well-structured subspaces and by analysing these subspaces we are able to identify all the irreducible invariant subspaces of $\mathfrak{g}_{\mathrm{IMM}}$. 


\subsection{Structure of the Lie algebra $\mathfrak{g}_{\mathrm{MMM}}$}

Recall that $\mathbf{x}=\mathbf{x}_{1} \uplus \mathbf{x}_{2} \uplus \cdots \uplus \mathbf{x}_{d}$ are the variables of IMM which are also referred to as $\left\{x_{1}, x_{2}, \ldots, x_{n}\right\}^{28}$ for notational convenience.

- Lemma 31. Let $\mathcal{W}_{1}, \mathcal{W}_{2}, \mathcal{W}_{3}$ be the following sets (spaces) of matrices:

1. $\mathcal{W}_{1}$ consists of all matrices $D=\left(d_{i j}\right)_{i, j \in[n]}$ such that $D$ is diagonal and

$$
\sum_{i=1}^{n} d_{i i} x_{i} \cdot \frac{\partial \mathrm{IMM}}{\partial x_{i}}=0
$$

2. $\mathcal{W}_{2}$ consists of all matrices $B=\left(b_{i j}\right)_{i, j \in[n]}$ such that

$$
\sum_{i, j \in[n]} b_{i j} x_{j} \cdot \frac{\partial \mathrm{IMM}}{\partial x_{i}}=0
$$

where in every summand $b_{i j} \neq 0$ only if $x_{i} \neq x_{j}$ and $x_{i}, x_{j} \in \mathbf{x}_{l}$ for some $l \in[d]$.

3. $\mathcal{W}_{3}$ consists of all matrices $C=\left(c_{i j}\right)_{i, j \in[n]}$ such that

$$
\sum_{i, j \in[n]} c_{i j} x_{j} \cdot \frac{\partial \mathrm{IMM}}{\partial x_{i}}=0
$$

where in every summand $c_{i j} \neq 0$ only if either $x_{i} \in \mathbf{x}_{2}, x_{j} \in \mathbf{x}_{1}$ or $x_{i} \in \mathbf{x}_{d-1}, x_{j} \in \mathbf{x}_{d}$. Then $\mathfrak{g}_{\mathrm{IMM}}=\mathcal{W}_{1} \oplus \mathcal{W}_{2} \oplus \mathcal{W}_{3}$.

The proof of Lemma 31 is given in Section 7.2.

Elaboration on Lemma 31: An element $E=\left(e_{i j}\right)_{i, j \in[n]}$ of $\mathfrak{g}_{\mathrm{IMM}}$ is an $n \times n$ matrix with rows and columns indexed by variables of IMM following the ordering mentioned in Section 2.3. Since $\sum_{i, j \in[n]} e_{i j} x_{j} \cdot \frac{\partial \mathrm{IMM}}{\partial x_{i}}=0, E$ appears as shown in Figure 2, where the row indices correspond to derivatives and column indices correspond to shifts ${ }^{29}$.

The proof will show that $E$ is a sum of three matrices $D \in \mathcal{W}_{1}, B \in \mathcal{W}_{2}$ and $C \in \mathcal{W}_{3}$ such that

1. $D$ contributes to the diagonal entries.

2. $B$ contributes to the block-diagonal entries of $E$ corresponding to the locations:

- $\left(x_{i}^{(1)}, x_{j}^{(1)}\right)$ where $i, j \in\left[w_{1}\right]$ and $i \neq j$

- $\left(x_{i}^{(d)}, x_{j}^{(d)}\right)$ where $i, j \in\left[w_{d-1}\right]$ and $i \neq j$

- $\left(x_{i j}^{(l)}, x_{p q}^{(l)}\right)$ where $i, p \in\left[w_{l-1}\right]$ and $j, q \in\left[w_{l}\right]$ for $l \in[2, d-1]$, and $(i, j) \neq(p, q)$.

3. $C$ contributes to the two corner rectangular blocks corresponding to:

- rows labelled by $\mathbf{x}_{2}$ variables and columns labelled by $\mathbf{x}_{1}$ variables

- rows labelled by $\mathbf{x}_{d-1}$ variables and columns labelled by $\mathbf{x}_{d}$ variables.

In order to get a finer understanding of $\mathfrak{g}_{\mathrm{IMM}}$ and its dimension we look at the spaces $\mathcal{W}_{1}, \mathcal{W}_{2}$ and $\mathcal{W}_{3}$ closely, and henceforth call them the diagonal space, the block-diagonal space and the corner space respectively.

\footnotetext{
${ }^{28}$ Following the ordering mentioned in Section 2.3 .

${ }^{29}$ Borrowing terminology from the shifted partial derivatives measure [26].
} 


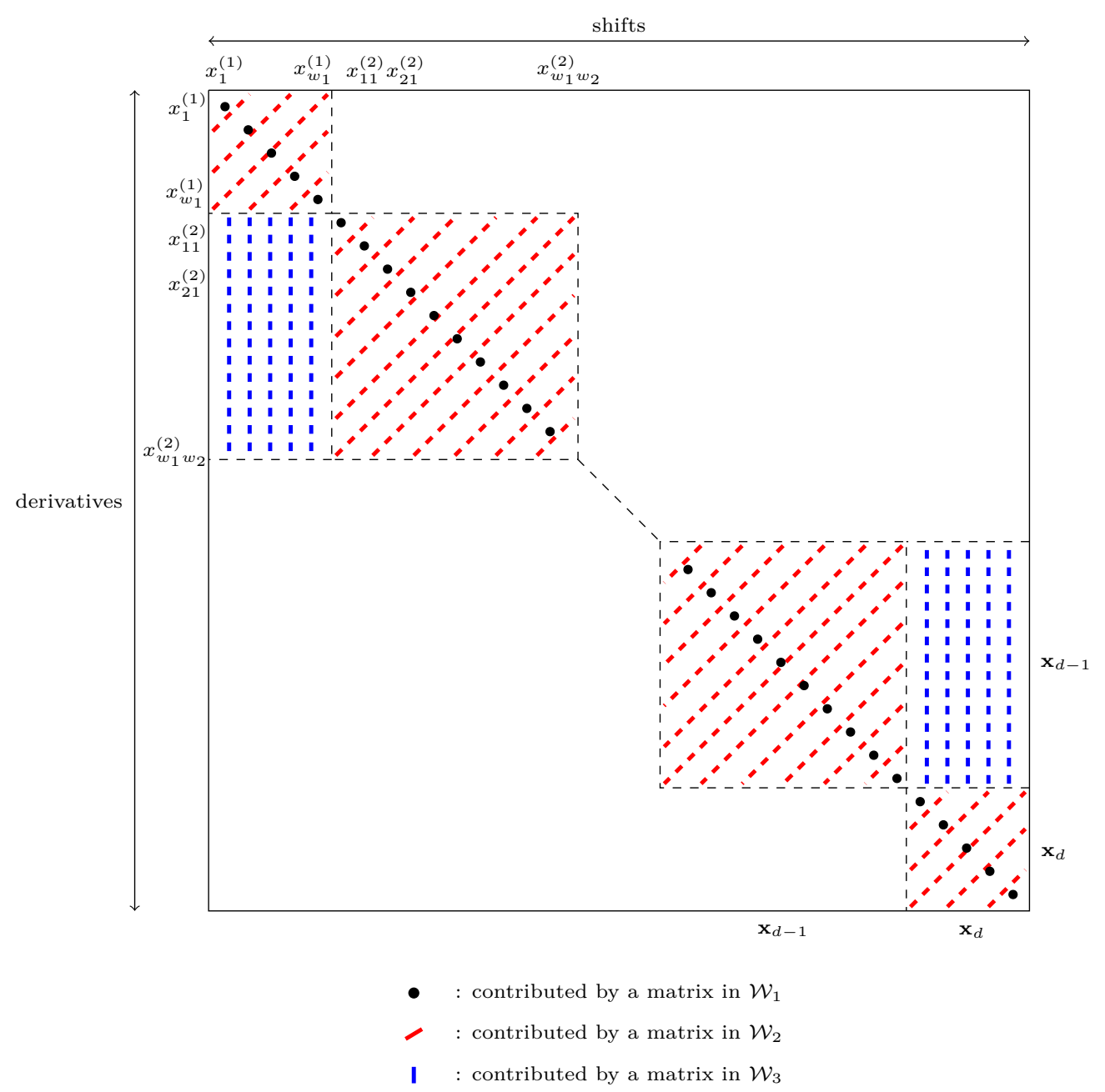

Figure 2 A matrix $E$ in $\mathfrak{g}_{\mathrm{IMM}}$.

\section{Corner space $\mathcal{W}_{3}$}

- Lemma 32 (Corner space). The space $\mathcal{W}_{3}=\mathcal{W}_{3}^{(a)} \oplus \mathcal{W}_{3}^{(b)}$ where $\mathcal{W}_{3}^{(a)}=\mathcal{A}_{1} \oplus \mathcal{A}_{2} \oplus \cdots \oplus \mathcal{A}_{w_{2}}$ and $\mathcal{W}_{3}^{(b)}=\mathcal{A}^{\prime}{ }_{1} \oplus \mathcal{A}^{\prime}{ }_{2} \oplus \cdots \oplus \mathcal{A}_{w_{d-2}}^{\prime}$ such that for every $i \in\left[w_{2}\right] \mathcal{A}_{i}$ is isomorphic to the space of $w_{1} \times w_{1}$ anti-symmetric matrices over $\mathbb{F}$, and for every $j \in\left[w_{d-2}\right] \mathcal{A}^{\prime}{ }_{j}$ is isomorphic to the space of $w_{d-1} \times w_{d-1}$ anti-symmetric matrices over $\mathbb{F}$. Hence $\operatorname{dim}\left(\mathcal{W}_{3}\right)=$ $\frac{1}{2}\left[w_{1} w_{2}\left(w_{1}-1\right)+w_{d-1} w_{d-2}\left(w_{d-1}-1\right)\right]$.

The proof is in Section 7.2. We briefly elaborate on the statement here.

Elaboration on Lemma 31: Every element $C \in \mathcal{W}_{3}$ can be expressed as a sum of two $n \times n$ matrices $C^{(a)} \in \mathcal{W}_{3}^{(a)}$ and $C^{(b)} \in \mathcal{W}_{3}^{(b)}$. $C^{(a)}$ looks as shown in Figure 3, where for every $i \in\left[w_{2}\right] C_{i}^{(a)}$ is an anti-symmetric matrix. The structure of $C^{(b)}$ is similar ${ }^{30}$ to that of $C^{(a)}$

\footnotetext{
${ }^{30}$ Once we rearrange the rows in $C^{(b)}$ indexed by variables in $\mathbf{x}_{d-1}$ according to row major ordering (instead of column major ordering) of variables in $\mathbf{x}_{d-1}$.
} 


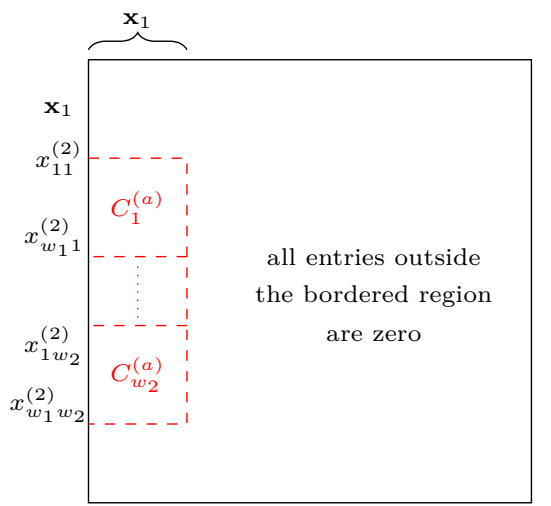

Figure 3 A matrix $C^{(a)}$ in $\mathcal{W}_{3}^{(a)}$.

with non zero entries restricted to the rows indexed by $\mathbf{x}_{d-1}$ variables and columns indexed by $\mathbf{x}_{d}$ variables.

\section{Block-diagonal space $\mathcal{W}_{2}$}

In the following lemma, $\mathcal{Z}_{w_{k}}$ denotes the space of $w_{k} \times w_{k}$ matrices with diagonal entries zero for $k \in[d-1]$. Also, for notational convenience we assume that $w_{0}=w_{d}=1$. We will also use the tensor product of matrices: if $A=\left(a_{i, j}\right) \in \mathbb{F}^{r \times s}$ and $B \in \mathbb{F}^{t \times u}$, then $A \otimes B$ is the $(r t) \times(s u)$ matrix given by

$$
A \otimes B=\left[\begin{array}{ccc}
a_{1,1} B & \cdots & a_{1, s} B \\
\vdots & \vdots & \vdots \\
a_{r, 1} B & \cdots & a_{r, s} B
\end{array}\right] .
$$

- Lemma 33 (Block-diagonal space). The space $\mathcal{W}_{2}=\mathcal{B}_{1} \oplus \mathcal{B}_{2} \oplus \cdots \oplus \mathcal{B}_{d-1}$ such that for every $k \in[d-1], \mathcal{B}_{k}$ is isomorphic to the $\mathbb{F}$-linear space spanned by $t_{k} \times t_{k}$ matrices of the form

$$
\left[\begin{array}{cc}
-Z^{T} \otimes I_{w_{k-1}} & 0 \\
0 & I_{w_{k+1}} \otimes Z
\end{array}\right]_{t_{k} \times t_{k}} \quad \text { where } Z \in \mathcal{Z}_{w_{k}} \text { and } t_{k}=w_{k}\left(w_{k-1}+w_{k+1}\right) .
$$

Hence, $\operatorname{dim}\left(\mathcal{W}_{2}\right)=\sum_{k=1}^{d-1}\left(w_{k}^{2}-w_{k}\right)$

The proof is in Section 7.2.

Elaboration on Lemma 33: An element $B \in \mathcal{W}_{2}$ is a sum of $d-1, n \times n$ matrices $B_{1}, B_{2}, \ldots$, $B_{d-1}$ such that for every $k \in[d-1], B_{k} \in \mathcal{B}_{k}$ and the non zero entries of $B_{k}$ are restricted to the rows and columns indexed by $\mathbf{x}_{k} \uplus \mathbf{x}_{k+1}$ variables. The submatrix in $B_{k}$ corresponding to these rows and columns looks as shown in Equation (1).

\section{Diagonal space $\mathcal{W}_{1}$}

In the next lemma, $\mathcal{Y}_{w_{k}}$ denotes the space of $w_{k} \times w_{k}$ diagonal matrices for $k \in[d-1]$. As before we assume $w_{0}=w_{d}=1$. 
- Lemma 34 (Diagonal Space). The space $\mathcal{W}_{1}$ contains the space $\mathcal{D}_{1} \oplus \mathcal{D}_{2} \oplus \cdots \oplus \mathcal{D}_{d-1}$ such that for every $k \in[d-1], \mathcal{D}_{k}$ is isomorphic to the $\mathbb{F}$-linear space spanned by $t_{k} \times t_{k}$ matrices of the form

$$
\left[\begin{array}{cc}
-Y \otimes I_{w_{k-1}} & 0 \\
0 & I_{w_{k+1}} \otimes Y
\end{array}\right]_{t_{k} \times t_{k}} \quad \text { where } Y \in \mathcal{Y}_{w_{k}} \text { and } t_{k}=w_{k}\left(w_{k-1}+w_{k+1}\right)
$$

Hence, $\operatorname{dim}\left(\mathcal{W}_{1}\right) \geq \sum_{k=1}^{d-1} w_{k}$.

The proof (still given in Section 7.2) is similar to that of Lemma 33.

Elaboration on Lemma 34: An element $D \in \mathcal{D}_{1} \oplus \mathcal{D}_{2} \oplus \cdots \oplus \mathcal{D}_{d-1}$ is a sum of $d-1, n \times n$ matrices $D_{1}, D_{2}, \ldots, D_{d-1}$ such that for every $k \in[d-1], D_{k} \in \mathcal{D}_{k}$ and the non zero entries of $D_{k}$ are restricted to the rows and columns indexed by $\mathbf{x}_{k} \uplus \mathbf{x}_{k+1}$ variables. The submatrix in $D_{k}$ corresponding to these rows and columns looks as shown in Equation (2).

\subsection{Random elements of $\mathfrak{g}_{\mathrm{MM}}$}

The algorithm in Theorem 7 involves picking a random matrix $R^{\prime}$ in $\mathfrak{g}_{f}$ and computing its characteristic polynomial $h(x)$. To ensure the correctness of the algorithm, $h(x)$ will have to be square free over $\mathbb{F}$. In Lemma 36 we show that the characteristic polynomial of a random matrix $R$ in $\mathfrak{g}_{\mathrm{IMM}}$ is square free with high probability. From Claim 21 this implies that if $f$ is equivalent to IMM then the characteristic polynomial of $R^{\prime}$ is also square free with high probability.

- Claim 35. There is a diagonal matrix $D \in \mathfrak{g}_{\mathrm{IMM}}$ with all entries distinct.

Proof. From Lemma 34, we know that for $k \in[d-1]$ the submatrix of $D_{k} \in \mathcal{D}_{k}$ defined by the rows and columns indexed by the variables in $\mathbf{x}_{k} \uplus \mathbf{x}_{k+1}$ is

$$
\left[\begin{array}{cc}
-Y_{k} \otimes I_{w_{k-1}} & 0 \\
0 & I_{w_{k+1}} \otimes Y_{k}
\end{array}\right]
$$

where $Y_{k} \in \mathcal{Y}_{k}$. Let the $(i, i)$-th entry of $Y_{k}$ be $y_{i}^{(k)}$ and pretend that these entries are distinct formal variables, say $\mathbf{y}$ variables. Consider the matrix $D=\sum_{i=1}^{d-1} D_{i}$ and observe the following:

(a) For $k \in[2, d-1]$, the $\left(x_{i j}^{(k)}, x_{i j}^{(k)}\right)$-th entry of $D$ is $y_{i}^{(k-1)}-y_{j}^{(k)}$ where $i \in\left[w_{k-1}\right]$ and $j \in\left[w_{k}\right]$

(b) The $\left(x_{i}^{(1)}, x_{i}^{(1)}\right)$-th and $\left(x_{j}^{(d)}, x_{j}^{(d)}\right)$-th entry of $D$ are $-y_{i}^{(1)}$ and $y_{j}^{(d-1)}$ respectively, where $i \in\left[w_{1}\right]$ and $j \in\left[w_{d-1}\right]$.

In particular, all the diagonal entries of $D$ are distinct linear forms in the $\mathbf{y}$ variables. Hence, if we assign values to the $\mathbf{y}$ variables uniformly at random from a set $S \subseteq \mathbb{F}$ such that $|S| \geq n^{2}$ then with non zero probability $D$ has all diagonal entries distinct after the random assignment.

- Lemma 36. If $\left\{L_{1}, L_{2}, \ldots, L_{m}\right\}$ is a basis of the Lie algebra $\mathfrak{g}_{\mathrm{MM}}$ then the characteristic polynomial of an element $L=\sum_{i=1}^{m} r_{i} L_{i}$, where $r_{i} \in_{R} \mathbb{F}$ is picked independently and uniformly at random from $\left[2 n^{3}\right]$, is square free with probability at least $1-\frac{1}{\operatorname{poly}(n)}$.

Proof. Pretend that the $r_{i}$ 's are formal variables. The characteristic polynomial $h_{\mathbf{r}}(x)$ of $L$ is a polynomial in $x$ with coefficients that are polynomial of degree at most $n$ in $\mathbf{r}=\left\{r_{1}, r_{2}, \ldots, r_{m}\right\}$ variables. 
- Observation 37. The discriminant of $h_{\mathbf{r}}(x), \operatorname{disc}\left(h_{\mathbf{r}}(x)\right):=\operatorname{res}_{x}\left(h_{\mathbf{r}}, \frac{\partial h_{\mathbf{r}}}{\partial x}\right)$, is a non zero polynomial in $\mathbf{r}$ variables of degree at most $n^{2}$, where $\operatorname{res}_{x}\left(h_{\mathbf{r}}, \frac{\partial h_{\mathbf{r}}}{\partial x}\right)$ is the resultant of $h_{\mathbf{r}}$ and $\frac{\partial h_{\mathbf{r}}}{\partial x}$ when treated as univariates in $x$.

Proof. $h_{\mathbf{r}}=\operatorname{Det}\left(x I_{n}-r_{1} L_{1}-\ldots-r_{\omega} L_{\omega}\right)$ is a degree $n$ homogeneous polynomial in the variables $x, r_{1}, \ldots, r_{\omega}$. Let $S \in \mathbb{F}[\mathbf{r}]^{(2 n-1)^{2}}$ be the Sylvester matrix of $h_{\mathbf{r}}$ and $\frac{\partial h_{\mathbf{r}}}{\partial x}$ with respect to $x$, i.e.

$$
S_{i, j}= \begin{cases}{\left[x^{n+i-j}\right] h_{\mathbf{r}}} & \text { if } 1 \leq i \leq n-1 \\ {\left[x^{i-j}\right] \frac{\partial h_{\mathbf{r}}}{\partial x}} & \text { otherwise }\end{cases}
$$

where $\left[x^{\delta}\right] g$ is the coefficient of the monomial $x^{\delta}$ in the polynomial $g$. Moreover, by homogeneity of $h_{\mathbf{r}},\left[x^{\delta}\right] h_{\mathbf{r}}$ (resp. $\left[x^{\delta}\right] \frac{\partial h_{\mathbf{r}}}{\partial x}$ ) is a homogeneous polynomial of degree $(n-\delta)$ (resp. $(n-1-\delta))$ with respect to the variables $\mathbf{r}$. Then, if $\sigma$ is a permutation of $[2 n-1]$ then $\prod_{i=1}^{2 n-1} S_{i, \sigma(i)}$ is a homogeneous polynomial in $\mathbf{r}$ of degree

$$
\sum_{i=1}^{n-1}-i+\sigma(i)+\sum_{i=n}^{2 n-1} n-1-i+\sigma(i)=n(n-1)-\left(\sum_{i=1}^{2 n-1} i\right)+\left(\sum_{i=1}^{2 n-1} \sigma(i)\right)=n(n-1) .
$$

Consequently, $\operatorname{disc}\left(h_{\mathbf{r}}(x)\right)$ is homogeneous and of degree $n(n-1)$. If $\operatorname{res}_{x}\left(h_{\mathbf{r}}, \frac{\partial h_{\mathbf{r}}}{\partial x}\right)$ is identically zero as a polynomial in $\mathbf{r}$ then for every setting of $\mathbf{r}$ to field elements $\operatorname{gcd}\left(h_{\mathbf{r}}, \frac{\partial h_{\mathbf{r}}}{\partial x}\right) \neq 1$ implying $h_{\mathbf{r}}$ is not square free. This would contradict Claim 35 as we can set the $\mathbf{r}$ variables appropriately such that $L$ is a diagonal matrix with distinct diagonal entries, and $h_{\mathbf{r}}$ for such a setting of the $\mathbf{r}$ variables is square free.

Since $\operatorname{disc}\left(h_{\mathbf{r}}(x)\right)$ is not an identically zero polynomial in the $\mathbf{r}$ variables and has degree less than $2 n^{2}$, if we set every $\mathbf{r}$ variable uniformly and independently at random to a value in $\left[2 n^{3}\right]$ then using Schwartz-Zippel lemma with probability at least $1-\frac{1}{\operatorname{poly}(n)}, \operatorname{gcd}\left(h_{\mathbf{r}}, \frac{\partial h_{\mathbf{r}}}{\partial x}\right)=1$. This implies with probability at least $1-\frac{1}{\operatorname{poly}(n)}, h_{\mathbf{r}}(x)$ is square free.

\subsection{Invariant subspaces of $\mathfrak{g}_{\mathrm{MM}}$}

The ordering of the variables in IMM allows us to identify them naturally with the unit vectors $e_{1}, e_{2}, \ldots, e_{n}$ in $\mathbb{F}^{n}$ - the vector $e_{i}$ corresponds to the $i$-th variable in the ordering. We will write $e_{x}$ to refer to the unit vector corresponding to the variable $x$. Let $\mathcal{U}_{1,2}$ represent the coordinate subspace spanned by the unit vectors corresponding to the variables in $\mathbf{x}_{1} \uplus \mathbf{x}_{2}$. Similarly $\mathcal{U}_{k}$ represents the coordinate subspace spanned by the unit vectors corresponding to the variables in $\mathbf{x}_{k}$ for $k \in[2, d-1]$, and $\mathcal{U}_{d-1, d}$ represents the coordinate subspace spanned by the unit vectors corresponding to the variables in $\mathbf{x}_{d-1} \uplus \mathbf{x}_{d}$. In Lemma 39, we establish that $\mathcal{U}_{1,2}, \mathcal{U}_{2}, \ldots, \mathcal{U}_{d-1}, \mathcal{U}_{d-1, d}$ are the only irreducible invariant subspaces of $\mathfrak{g}_{\text {IMм }}$.

- Claim 38. Let $\mathcal{U}$ be a nonzero invariant subspace of $\mathfrak{g}_{\mathrm{Mм}}$. If $\mathbf{u}=\left(u_{1}, u_{2}, \ldots, u_{n}\right)^{T} \in \mathcal{U}$ and $u_{j} \neq 0$ then $e_{j} \in \mathcal{U}$, implying $\mathcal{U}$ is a coordinate subspace.

Proof. Claim 35 states that there is a diagonal matrix $D \in \mathfrak{g}_{\mathrm{MM}}$ with distinct diagonal entries $\lambda_{1}, \lambda_{2}, \ldots, \lambda_{n}$. Since $\mathcal{U}$ is invariant for $D$, if $\mathbf{u}=\left(u_{1}, u_{2}, \ldots, u_{n}\right)^{T} \in \mathcal{U}$ then $\left(\lambda_{1}^{i} u_{1}, \lambda_{2}^{i} u_{2}, \ldots\right.$, $\left.\lambda_{n}^{i} u_{n}\right) \in \mathcal{U}$ for every $i \in \mathbb{N}$. Let $S_{\mathbf{u}}:=\left\{j \in[n] \mid u_{j} \neq 0\right\}$ be the support of $\mathbf{u} \neq 0$. As $\lambda_{1}, \lambda_{2}, \ldots, \lambda_{n}$ are distinct, the vectors $\left(\lambda_{1}^{i} u_{1}, \lambda_{2}^{i} u_{2}, \ldots, \lambda_{n}^{i} u_{n}\right)$ are $\mathbb{F}$-linearly independent for $0 \leq i<\left|S_{\mathbf{u}}\right|$. Hence, the unit vector $e_{j} \in \mathcal{U}$ for every $j \in S_{\mathbf{u}}$. It follows that $\mathcal{U}$ is the coordinate subspace spanned by those $e_{j}$ for which $j \in S_{\mathbf{u}}$ for some $\mathbf{u} \in \mathcal{U}$. 
- Lemma 39. The only irreducible invariant subspaces of $\mathfrak{g}_{\mathrm{Iмм}}$ are $\mathcal{U}_{1,2}, \mathcal{U}_{2}, \ldots, \mathcal{U}_{d-1}$, $\mathcal{U}_{d-1, d}$.

Proof. It follows from Lemma 31 and Figure 2 that $\mathcal{U}_{1,2}, \mathcal{U}_{2}, \ldots, \mathcal{U}_{d-1}, \mathcal{U}_{d-1, d}$ are invariant subspaces. We show in the next two claims that the spaces $\mathcal{U}_{1,2}, \mathcal{U}_{2}, \ldots, \mathcal{U}_{d-1}, \mathcal{U}_{d-1, d}$ are irreducible. The proofs are given in Section 7.2.

- Claim 40. No invariant subspace of $\mathfrak{g}_{\mathrm{IMM}}$ is properly contained in $\mathcal{U}_{k}$ for $k \in[2, d-1]$.

Claim 41. The invariant subspaces $\mathcal{U}_{1,2}$ and $\mathcal{U}_{d-1, d}$ are irreducible, and the only invariant subspace properly contained in $\mathcal{U}_{1,2}$ (respectively $\mathcal{U}_{d-1, d}$ ) is $\mathcal{U}_{2}$ (respectively $\mathcal{U}_{d-1}$ ).

We in fact show in the proof of Claim 40 that the closure of $e_{x}$ under the action of $\mathfrak{g}_{\mathrm{Im}}$ is $\mathcal{U}_{k}$ for any $x \in \mathbf{x}_{k}$, where $k \in[2, d-1]$. Similarly, in the proof of Claim 41 we show that the closure of $e_{x}$ under the action of $\mathfrak{g}_{\mathrm{IMM}}$ is $\mathcal{U}_{1,2}$ (respectively $\mathcal{U}_{d-1, d}$ ) for any $x \in \mathbf{x}_{1}$ (respectively $\left.x \in \mathbf{x}_{d}\right)$. This observation helps infer that the spaces $\mathcal{U}_{1,2}, \mathcal{U}_{2}, \ldots, \mathcal{U}_{d-1}, \mathcal{U}_{d-1, d}$ are the only irreducible invariant subspaces of $\mathfrak{g}_{\mathrm{IMM}}$ : Suppose $\mathcal{V}$ is an irreducible invariant subspace. If $e_{x} \in \mathcal{V}$ for some $x \in \mathbf{x}_{k}$ where $k \in[2, d-1]$, then $\mathcal{U}_{k} \subseteq \mathcal{V}$ as $\mathcal{U}_{k}$ is the closure of $e_{x}$. If $e_{x} \in \mathcal{V}$ for some $x \in \mathbf{x}_{1}$ (respectively $x \in \mathbf{x}_{d}$ ) then $\mathcal{U}_{1,2} \subseteq \mathcal{V}$ (respectively $\mathcal{U}_{d-1, d} \subseteq \mathcal{V}$ ) as $\mathcal{U}_{1,2}$ (respectively $\mathcal{U}_{d-1, d}$ ) is the closure of $e_{x}$. Therefore $\mathcal{V}$ is a direct sum of some of the irreducible invariant subspaces $\mathcal{U}_{1,2}, \mathcal{U}_{2}, \ldots, \mathcal{U}_{d-1}, \mathcal{U}_{d-1, d}$. Since $\mathcal{V}$ is irreducible, it is equal to one of these irreducible invariant subspaces.

- Corollary 42 (Uniqueness of decomposition). The decomposition,

$$
\mathbb{F}^{n}=\mathcal{U}_{1,2} \oplus \mathcal{U}_{3} \oplus \cdots \oplus \mathcal{U}_{d-2} \oplus \mathcal{U}_{d-1, d}
$$

is unique in the following sense; if $\mathbb{F}^{n}=\mathcal{V}_{1} \oplus \mathcal{V}_{2} \oplus \cdots \oplus \mathcal{V}_{s}$, where $\mathcal{V}_{i}^{\prime} s$ are irreducible invariant subspaces of $\mathfrak{g}_{\mathrm{IMM}}$, then $s=d-2$ and for every $i \in[s], \mathcal{V}_{i}$ is equal to $\mathcal{U}_{1,2}$ or $\mathcal{U}_{d-1, d}$, or some $\mathcal{U}_{k}$ for $k \in[3, d-2]$.

Proof. Since $\mathcal{V}_{i}$ 's are irreducible invariant subspaces, from Lemma 39 it follows that for every $i \in[s] \mathcal{V}_{i}$ equals one among $\mathcal{U}_{1,2}, \mathcal{U}_{2}, \ldots, \mathcal{U}_{d-1}, \mathcal{U}_{d-1, d}$. Since $\mathcal{V}_{1}, \mathcal{V}_{2}, \ldots, \mathcal{V}_{s}$ span the entire $\mathbb{F}^{n}$, the only possible decomposition is $\mathbb{F}^{n}=\mathcal{U}_{1,2} \oplus \mathcal{U}_{3} \oplus \cdots \oplus \mathcal{U}_{d-2} \oplus \mathcal{U}_{d-1, d}$.

\section{Lie algebra of $f$ equivalent to IMM}

Let $f$ be an $n$ variate polynomial such that $f=\mathrm{IMM}_{\mathbf{w}, d}(A \mathbf{x})$, where $\mathbf{w}=\left(w_{1}, w_{2}, \ldots, w_{d-1}\right)$ $\in \mathbb{N}^{d-1}$ and $A \in \mathrm{GL}(n)$. It follows, $n=w_{1}+\sum_{i=2}^{d-1} w_{i-1} w_{i}+w_{d-1}$. From Observation 22 and Lemma 39 we know $A^{-1} \mathcal{U}_{1,2}, A^{-1} \mathcal{U}_{2}, \ldots, A^{-1} \mathcal{U}_{d-1}, A^{-1} \mathcal{U}_{d-1, d}$ are the only irreducible invariant subspaces of $\mathfrak{g}_{f}$, and $A^{-1} \mathcal{U}_{2}$ (respectively $A^{-1} \mathcal{U}_{d-1}$ ) is the only invariant subspace properly contained in $A^{-1} \mathcal{U}_{1,2}$ (respectively $A^{-1} \mathcal{U}_{d-1, d}$ ). Also from Corollary 42 it follows that $\mathbb{F}^{n}=A^{-1} \mathcal{U}_{1,2} \oplus A^{-1} \mathcal{U}_{3} \oplus \cdots \oplus A^{-1} \mathcal{U}_{d-2} \oplus A^{-1} \mathcal{U}_{d-1, d}$. In this section, we give an efficient randomized algorithm to compute a basis of each of the spaces $A^{-1} \mathcal{U}_{1,2}, A^{-1} \mathcal{U}_{2}, \ldots$, $A^{-1} \mathcal{U}_{d-1}, A^{-1} \mathcal{U}_{d-1, d}$ given only blackbox access to $f$ (but no knowledge of $\mathbf{w}$ or $A$ ).

\subsection{Computing invariant subspaces of the Lie algebra $\mathfrak{g}_{f}$}

First, we efficiently compute a basis $\left\{L_{1}^{\prime}, L_{2}^{\prime}, \ldots, L_{m}^{\prime}\right\}$ of $\mathfrak{g}_{f}$ using the algorithm stated in Lemma 28. By Claim 21, $L_{1}=A L_{1}^{\prime} A^{-1}, L_{2}=A L_{2}^{\prime} A^{-1}, \ldots, L_{m}=A L_{m}^{\prime} A^{-1}$ form a basis of $\mathfrak{g}_{\mathrm{MM}}$. Suppose $R^{\prime}=\sum_{i=1}^{m} r_{i} L_{i}^{\prime}$ is a random element of $\mathfrak{g}_{f}$, chosen by picking the $r_{i}$ 's independently and uniformly at random from $\left[2 n^{3}\right]$. Then $R=A R^{\prime} A^{-1}=\sum_{i=1}^{m} r_{i} L_{i}$ is a 


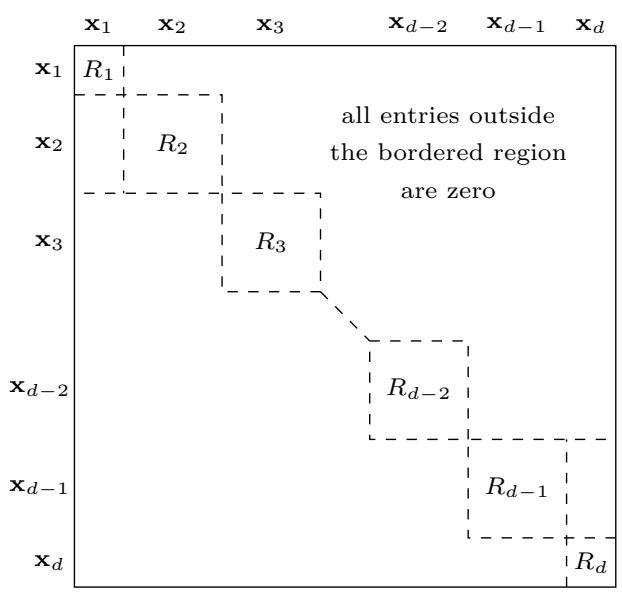

Figure 4 Random element $R$ in $\mathfrak{g}_{\mathrm{IMM}}$.

random element of $\mathfrak{g}_{\text {Імм }}$ and it follows from Lemma 36 that the characteristic polynomial of $R$ is square free with probability at least $1-\frac{1}{\operatorname{poly}(n)}$. So assume henceforth that the characteristic polynomial of $R$ (and hence also of $R^{\prime}$ ) is square free.

Moreover, from Figure 2 it follows that $R$ has the structure as shown in Figure 4.

Let $h(x)=\prod_{i=1}^{d} h_{i}(x)$ be the characteristic polynomial of $R$ and $R^{\prime}$, where $h_{i}(x)$ is the characteristic polynomial of $R_{i}$, and $g_{1}(x), g_{2}(x), \ldots, g_{s}(x)$ be the distinct irreducible factors of $h(x)$ over $\mathbb{F}$. Suppose $\mathcal{N}_{i}^{\prime}$ is the null space of $g_{i}\left(R^{\prime}\right)$. Thus $\mathcal{N}_{i}$, the null space of $g_{i}(R)$ (equal to $A \cdot g_{i}\left(R^{\prime}\right) \cdot A^{-1}$ ), is $A \mathcal{N}_{i}^{\prime}$ for $i \in[s]$. We study the null spaces $\mathcal{N}_{1}, \mathcal{N}_{2}, \ldots, \mathcal{N}_{s}$ in the next two claims and show how to extract out the irreducible invariant subspaces of $\mathfrak{g}_{f}$ from $\mathcal{N}_{1}^{\prime}, \mathcal{N}_{2}^{\prime}, \ldots, \mathcal{N}_{s}^{\prime}$ (as specified in Algorithm 3). The proofs of these claims (using simple linear algebra) can be found in Section 7.3.

- Claim 43. For all $i \in[s]$, let $\mathcal{N}_{i}$ and $\mathcal{N}_{i}^{\prime}$ be the null spaces of $g_{i}(R)$ and $g_{i}\left(R^{\prime}\right)$. Then:

1. $\mathbb{F}^{n}=\mathcal{N}_{1} \oplus \mathcal{N}_{2} \oplus \cdots \oplus \mathcal{N}_{s}=\mathcal{N}_{1}^{\prime} \oplus \mathcal{N}_{2}^{\prime} \oplus \cdots \oplus \mathcal{N}_{s}^{\prime}$.

2. For all $i \in[s], \operatorname{dim}\left(\mathcal{N}_{i}\right)=\operatorname{dim}\left(\mathcal{N}_{i}^{\prime}\right)=\operatorname{deg}_{x}\left(g_{i}\right)$.

- Claim 44. Suppose $g_{i}(x)$ is an irreducible factor of the characteristic polynomial $h_{k}(x)$ of $R_{k}$ (depicted in Figure 4) for some $k \in[d]$. Then the following holds:

1. If $k \in[2, d-1]$ then $\mathcal{N}_{i} \subseteq \mathcal{U}_{k}$ (equivalently $\mathcal{N}_{i}^{\prime} \subseteq A^{-1} \mathcal{U}_{k}$ ).

2. If $k=1$ then $\mathcal{N}_{i} \subseteq \mathcal{U}_{1,2}$ (equivalently $\mathcal{N}_{i}^{\prime} \subseteq A^{-1} \mathcal{U}_{1,2}$ ), and if $k=d$ then $\mathcal{N}_{i} \subseteq \mathcal{U}_{d-1, d}$ (equivalently $\left.\mathcal{N}_{i}^{\prime} \subseteq A^{-1} \mathcal{U}_{d-1, d}\right)$.

- Claim 45.

1. If $g_{l_{1}}(x), g_{l_{2}}(x), \ldots, g_{l_{r}}(x)$ are all the irreducible factors of $h_{k}(x)$ for $k \in[2, d-1]$ then $A^{-1} \mathcal{U}_{k}=\mathcal{N}_{l_{1}}^{\prime} \oplus \mathcal{N}_{l_{2}}^{\prime} \oplus \cdots \oplus \mathcal{N}_{l_{r}}^{\prime}$.

2. If $g_{l_{1}}(x), g_{l_{2}}(x), \ldots, g_{l_{r}}(x)$ are all the irreducible factors of $h_{1}(x) h_{2}(x)$ (respectively $h_{d-1}(x)$ $\left.h_{d}(x)\right)$ then $A^{-1} \mathcal{U}_{1,2}=\mathcal{N}_{l_{1}}^{\prime} \oplus \mathcal{N}_{l_{2}}^{\prime} \oplus \cdots \oplus \mathcal{N}_{l_{r}}^{\prime}$ (respectively $A^{-1} \mathcal{U}_{d-1, d}=\mathcal{N}_{l_{1}}^{\prime} \oplus \mathcal{N}_{l_{2}}^{\prime} \oplus \cdots \oplus \mathcal{N}_{l_{r}}^{\prime}$ ).

Proof. If $k \in[2, d-1]$ then $\mathcal{N}_{l_{1}}^{\prime}+\mathcal{N}_{l_{2}}^{\prime}+\cdots+\mathcal{N}_{l_{r}}^{\prime}$ is a direct sum and $\operatorname{dim}\left(A^{-1} \mathcal{U}_{k}\right)=\operatorname{deg}_{x}\left(h_{k}\right)=\sum_{j=1}^{r} \operatorname{deg}_{x}\left(g_{l_{j}}\right)=\sum_{j=1}^{r} \operatorname{dim}\left(\mathcal{N}_{l_{j}}^{\prime}\right)$, which follow from Claim 43.

Hence from Claim 44, $A^{-1} \mathcal{U}_{k}=\mathcal{N}_{l_{1}}^{\prime} \oplus \mathcal{N}_{l_{2}}^{\prime} \oplus \cdots \oplus \mathcal{N}_{l_{r}}^{\prime}$. The proof for the second part is similar. 


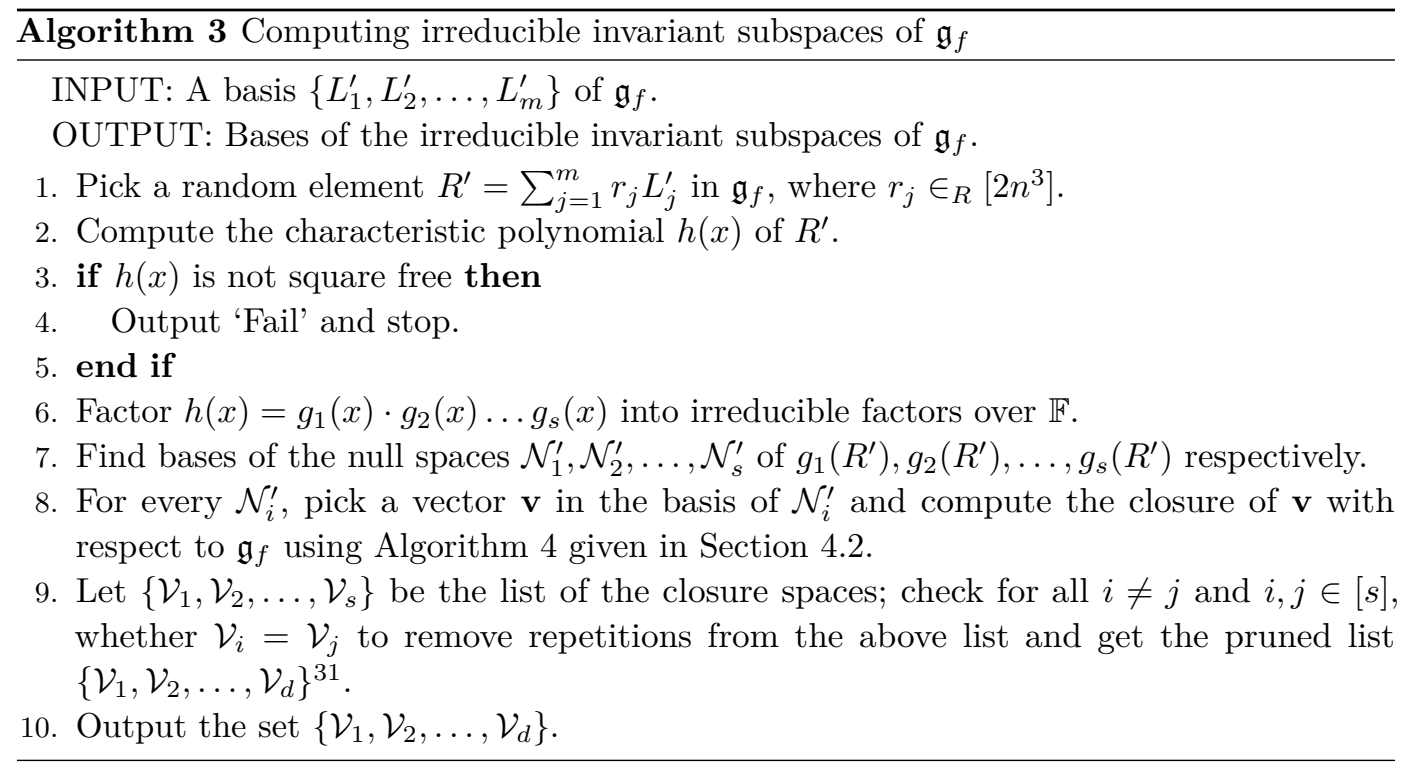

- Lemma 46. Given as input bases of the null spaces $\mathcal{N}_{1}^{\prime}, \mathcal{N}_{2}^{\prime}, \ldots, \mathcal{N}_{s}^{\prime}$ we can compute bases of the spaces $A^{-1} \mathcal{U}_{1,2}, A^{-1} \mathcal{U}_{2}, \ldots, A^{-1} \mathcal{U}_{d-1}, A^{-1} \mathcal{U}_{d-1, d}$ in deterministic polynomial time.

Proof. Recall $\mathcal{N}_{i}^{\prime}$ is the null space of $g_{i}\left(R^{\prime}\right)$, where $g_{i}(x)$ is an irreducible factor of $h_{k}(x)$ for some $k \in[d]$.

Case A: $\boldsymbol{k} \in[\mathbf{2}, \boldsymbol{d}-\mathbf{1}]$ : From Claim 44 it follows that $\mathcal{N}_{i}^{\prime} \subseteq A^{-1} \mathcal{U}_{k}$. Pick a basis vector $\mathbf{v}$ in $\mathcal{N}_{i}^{\prime}$ and compute the closure of $\mathbf{v}$ under the action of $\mathfrak{g}_{f}$ using Algorithm 4 given in Section 4.2. Since the closure of $\mathbf{v}$ is the smallest invariant subspace of $\mathfrak{g}_{f}$ containing $\mathbf{v}$, by Claim 40 the closure of $\mathbf{v}$ equals $A^{-1} \mathcal{U}_{k}$.

Case B: $\boldsymbol{k}=\mathbf{1}$ or $\boldsymbol{k}=\boldsymbol{d}$ : The arguments for $k=1$ and $k=d$ are similar. We prove it for $k=1$. From Claim 44 we have $\mathcal{N}_{i}^{\prime} \subseteq A^{-1} \mathcal{U}_{1,2}$. Pick a basis vector $\mathbf{v}$ of $\mathcal{N}_{i}^{\prime}$ and compute its closure under the action of $\mathfrak{g}_{f}$ using Algorithm 4. Similar to case A, this gives us an invariant subspace of $\mathfrak{g}_{f}$ contained in $A^{-1} \mathcal{U}_{1,2}$ and by Claim 41 this invariant subspace is either $A^{-1} \mathcal{U}_{2}$ or $A^{-1} \mathcal{U}_{1,2}$. However, $\mathcal{N}_{i}^{\prime} \cap A^{-1} \mathcal{U}_{2}$ (by Corollary 45) is empty, as $g_{i}(x)$ is an irreducible factor of $h_{1}(x)\left(\right.$ not $\left.h_{2}(x)\right)$. Hence $\mathbf{v} \notin A^{-1} \mathcal{U}_{2}$ and the closure of $\mathbf{v}$ must be $A^{-1} \mathcal{U}_{1,2}$.

To summarize, first we pick a random element $R^{\prime}$ in $\mathfrak{g}_{f}$, find its characteristic polynomial $h(x)$ and factorize $h(x)$ to get the irreducible factors $g_{1}(x), g_{2}(x), \ldots, g_{s}(x)$. Then we compute the null spaces $\mathcal{N}_{1}^{\prime}, \mathcal{N}_{2}^{\prime}, \ldots, \mathcal{N}_{s}^{\prime}$ of $g_{1}\left(R^{\prime}\right), g_{2}\left(R^{\prime}\right), \ldots, g_{s}\left(R^{\prime}\right)$ respectively. By applying Claim 46 , we find the invariant subspaces of $\mathfrak{g}_{f}, A^{-1} \mathcal{U}_{1,2}, A^{-1} \mathcal{U}_{2}, \ldots, A^{-1} \mathcal{U}_{d-1}, A^{-1} \mathcal{U}_{d-1, d}$ from these null spaces. We present this formally in Algorithm 3.

\section{Comments on Algorithm 3}

(a) Observe that in step 6 of the algorithm we need $\mathbb{F}$ to be $\mathbb{Q}$ (as assumed) or a finite field because univariate factorization can be done effectively over such fields [31, 9, 11].

\footnotetext{
${ }^{31}$ Reusing symbols.
} 


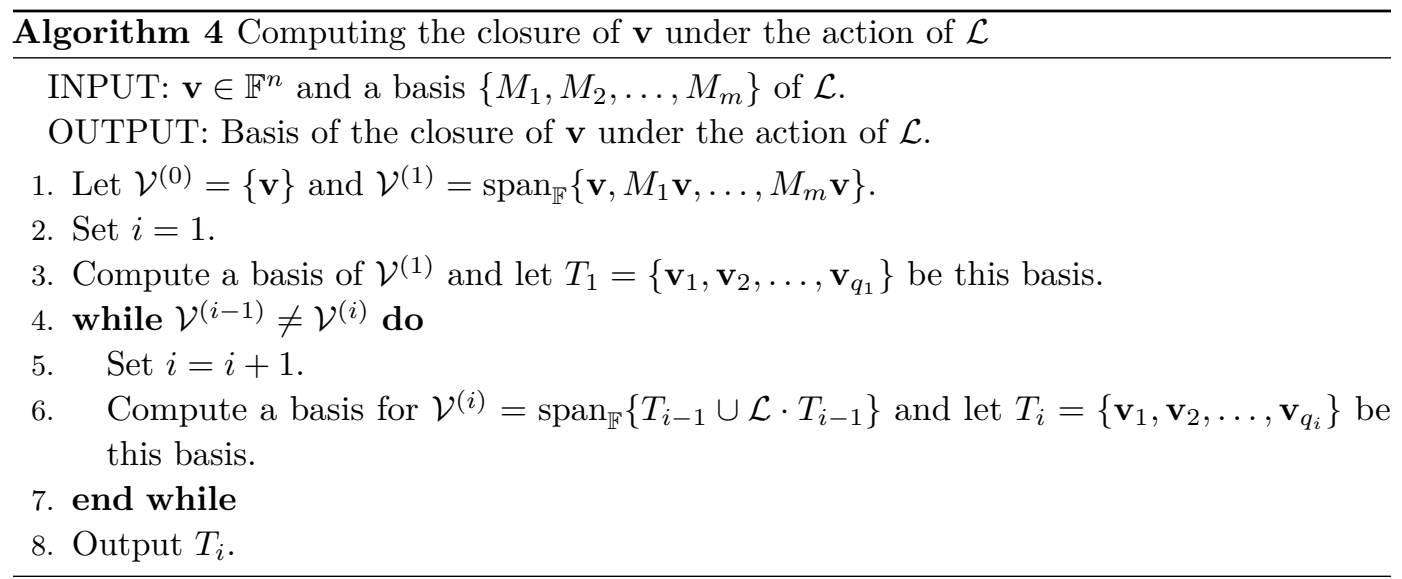

(b) When Algorithm 3 is invoked in Algorithm 2 for an $n$ variate degree $d$ polynomial $f$, there may not exists a $\mathbf{w} \in \mathbb{N}^{d-1}$ and an $A \in \mathrm{GL}(n)$ such that $f=\mathrm{IMM}_{\mathbf{w}, d}(A \mathbf{x})$. We point out a few additional checks that need to be added to the above algorithm to handle this case. In step 9, if the pruned list (after removing repetitions) has size other than $d$ then output 'Fail'. Also from Claim 41, exactly two subspaces in the pruned list $\left\{\mathcal{V}_{1}, \mathcal{V}_{2}, \ldots, \mathcal{V}_{d}\right\}$, say $\mathcal{V}_{2}$ and $\mathcal{V}_{d-1}$, should be subspaces of other vector spaces, say $\mathcal{V}_{1}$ and $\mathcal{V}_{d}$ respectively. We can find these two spaces by doing a pairwise check among the $d$ vector spaces. If such subspaces do not exist among $\mathcal{V}_{1}, \mathcal{V}_{2}, \ldots, \mathcal{V}_{d}$ then output 'Fail'. Further, if $\mathbb{F}^{n} \neq \mathcal{V}_{1} \oplus \mathcal{V}_{3} \oplus \cdots \oplus \mathcal{V}_{d-2} \oplus \mathcal{V}_{d}$ (assuming $\mathcal{V}_{2} \subseteq \mathcal{V}_{1}$ and $\mathcal{V}_{d-1} \subseteq \mathcal{V}_{d}$ ) then output 'Fail'.

(c) It follows from the above discussion, if $f=\mathrm{IMM}_{\mathbf{w}, d}(A \mathrm{x})$ then we can assume $\mathcal{V}_{3}, \mathcal{V}_{4}, \ldots$, $\mathcal{V}_{d-2}$ are the spaces $A^{-1} \mathcal{U}_{3}, A^{-1} \mathcal{U}_{4}, \ldots, A^{-1} \mathcal{U}_{d-2}$ in some unknown order. The spaces $\mathcal{V}_{1}, \mathcal{V}_{2}$ and $\mathcal{V}_{d}, \mathcal{V}_{d-1}$ are either the spaces $A^{-1} \mathcal{U}_{1,2}, A^{-1} \mathcal{U}_{2}$ and $A^{-1} \mathcal{U}_{d-1, d}, A^{-1} \mathcal{U}_{d-1}$ respectively, or the spaces $A^{-1} \mathcal{U}_{d-1, d}, A^{-1} \mathcal{U}_{d-1}$ and $A^{-1} \mathcal{U}_{1,2}, A^{-1} \mathcal{U}_{2}$ respectively.

\subsection{Closure of a vector under the action of $\mathfrak{g}_{f}$}

Algorithm 4 computes the closure of $\mathbf{v} \in \mathbb{F}^{n}$ under the action of a space $\mathcal{L}$ spanned by $n \times n$ matrices. Let $\left\{M_{1}, M_{2}, \ldots, M_{m}\right\}$ be a basis of $\mathcal{L}$ where $M_{i} \in \mathbb{F}^{n \times n}$. For a set of vectors $T=\left\{\mathbf{v}_{1}, \mathbf{v}_{2}, \ldots, \mathbf{v}_{q}\right\} \subseteq \mathbb{F}^{n}$, let $\mathcal{L} \cdot T$ denote the set $\left\{M_{a} \mathbf{v}_{b} \mid a \in[m]\right.$ and $\left.b \in[q]\right\}$.

- Claim 47. Algorithm 4 computes the closure of $\mathbf{v} \in \mathbb{F}^{n}$ under the action of $\mathcal{L}$ in time polynomial in $n$ and the bit length of the entries of $\mathbf{v}$ and $M_{1}, M_{2}, \ldots, M_{m}$.

Proof. The closure of $\mathbf{v}$ under the action of $\mathcal{L}$ is the $\mathbb{F}$-linear span of all vectors of the form $\mu$.v, where $\mu$ is a non-commutative monomial in $M_{1}, M_{2}, \ldots, M_{m}$ (including unity). Algorithm 4 computes exactly this set and hence the closure of $\mathbf{v}$. Moreover, $\operatorname{dim}\left(\mathcal{V}^{(i)}\right) \leq n$ and in every iteration of the while loop $\operatorname{dim}\left(\mathcal{V}^{(i)}\right)>\operatorname{dim}\left(\mathcal{V}^{(i-1)}\right)$, until $\mathcal{V}^{(i)}=\mathcal{V}^{(i-1)}$. Hence, Algorithm 4 runs in time polynomial in $n$ and the bit length of the entries of $\mathbf{v}$ and $M_{1}, M_{2}, \ldots, M_{m}$.

\section{Reconstruction of full rank ABP for $f$}

Let $f$ be a polynomial equivalent to $\mathrm{IMM}_{\mathbf{w}, d}$ for some (unknown) $\mathbf{w} \in \mathbb{N}^{d-1}$. In this section, we show that the invariant subspaces of $\mathfrak{g}_{f}$ let us compute a $\mathbf{w} \in \mathbb{N}^{d-1}$ and an $A \in \mathrm{GL}(n)$ such that $f=\mathrm{IMM}_{\mathbf{w}, d}(A \mathbf{x})$. Since $f$ is equivalent to $\mathrm{IMM}_{\mathbf{w}, d}$, it is computable by a full rank ABP $X_{1} \cdot X_{2} \ldots X_{d-1} \cdot X_{d}$ of width $\mathbf{w}$ and length $d$ with linear form entries in the 
matrices. We call this full rank ABP A which, as explained below, is not the only full rank ABP computing $f$.

Many full rank ABPs for $f$ : The full rank $\mathrm{ABP} X_{1}^{\prime} \cdot X_{2}^{\prime} \cdots X_{d}^{\prime}$ resulting from each of the following three transformations on A still computes $f$,

1. Transposition: Set $X_{k}^{\prime}=X_{d+1-k}^{T}$ for $k \in[d]$.

2. Left-right multiplications: Let $A_{1}, \ldots, A_{d-1}$ be matrices such that $A_{k} \in \mathrm{GL}\left(w_{k}\right)$ for every $k \in[d-1]$. Set $X_{1}^{\prime}=X_{1} \cdot A_{1}, X_{d}^{\prime}=A_{d-1}^{-1} \cdot X_{d}$, and $X_{k}^{\prime}=A_{k-1}^{-1} \cdot X_{k} \cdot A_{k}$ for $k \in[2, d-1]$.

3. Corner translations: Suppose $\left\{C_{11}, C_{12}, \ldots, C_{1 w_{2}}\right\}$ and $\left\{C_{d 1}, C_{d 2}, \ldots, C_{d w_{d-2}}\right\}$ are two sets containing anti-symmetric matrices in $\mathbb{F}^{w_{1} \times w_{1}}$ and $\mathbb{F}^{w_{d-1} \times w_{d-1}}$ respectively. Let $Y_{2} \in \mathbb{F}[\mathbf{x}]^{w_{1} \times w_{2}}$ (respectively $Y_{d-1} \in \mathbb{F}[\mathbf{x}]^{w_{d-2} \times w_{d-1}}$ ) be a matrix with its $i$-th column (respectively $i$-th row) equal to $C_{1 i} \cdot X_{1}^{T}$ (respectively $X_{d}^{T} \cdot C_{d i}$ ). Set $X_{2}^{\prime}=X_{2}+Y_{2}$, $X_{d-1}^{\prime}=X_{d-1}+Y_{d-1}$, and $X_{k}^{\prime}=X_{k}$ for $k \in[d] \backslash\{2, d-1\}$.

In each of the above three cases $f=X_{1}^{\prime} \cdot X_{2}^{\prime} \cdots X_{d}^{\prime}$; this is easy to verify for cases 1 and 2 , in case 3 observe that $X_{1} \cdot C_{1 i} \cdot X_{1}^{T}=X_{d}^{T} \cdot C_{d i} \cdot X_{d}=0$.

It turns out that the full rank ABPs obtained by (repeatedly) applying the above three transformations on A are the only full rank ABPs computing $f$. This would follow from the discussion in Section 6. Although there are multiple full rank ABPs for $f$, the layer spaces of these ABPs are unique (Lemma 48). This uniqueness of the layer spaces essentially facilitates the recovery of a full rank $\mathrm{ABP}$ for $f$. Let us denote the span of the linear forms ${ }^{32}$ in $X_{1}$ and $X_{2}$ (respectively $X_{d-1}$ and $X_{d}$ ) by $\mathcal{X}_{1,2}$ (respectively $\mathcal{X}_{d-1, d}$ ).

- Lemma 48 (Uniqueness of the layer spaces of full rank ABP for $f$ ). Suppose $X_{1} \cdot X_{2} \cdots X_{d}$ and $X_{1}^{\prime} \cdot X_{2}^{\prime} \cdots X_{d}^{\prime}$ are two full rank $A B P s$ of widths $\mathbf{w}=\left(w_{1}, w_{2}, \ldots, w_{d-1}\right)$ and $\mathbf{w}^{\prime}=$ $\left(w_{1}^{\prime}, w_{2}^{\prime}, \ldots, w_{d-1}^{\prime}\right)$ respectively, computing the same polynomial $f$. Then one of the following two cases is true:

(a) $w_{k}^{\prime}=w_{k}$ for $k \in[d-1]$, and the spaces $\mathcal{X}_{1}^{\prime}, \mathcal{X}_{1,2}^{\prime}, \mathcal{X}_{3}^{\prime}, \ldots, \mathcal{X}_{d-1, d}^{\prime}, \mathcal{X}_{d}^{\prime}$ are the spaces $\mathcal{X}_{1}, \mathcal{X}_{1,2}, \mathcal{X}_{3}, \ldots, \mathcal{X}_{d-1, d}, \mathcal{X}_{d}$ respectively.

(b) $w_{k}^{\prime}=w_{d-k}$ for $k \in[d-1]$, and the spaces $\mathcal{X}_{1}^{\prime}, \mathcal{X}_{1,2}^{\prime}, \mathcal{X}_{3}^{\prime}, \ldots, \mathcal{X}_{d-1, d}^{\prime}, \mathcal{X}_{d}^{\prime}$ are the spaces $\mathcal{X}_{d}, \mathcal{X}_{d-1, d}, \mathcal{X}_{d-2}, \ldots, \mathcal{X}_{1,2}, \mathcal{X}_{1}$ respectively.

The lemma would help characterize the group of symmetries of IMM in Section 6; the proof would follow readily from Claim 50 in Section 5.2. With an eye on Section 6 and for better clarity in the reduction to almost set-multilinear ABP in Section 5.2, we take a slight detour and show next how to compute these 'unique' layer spaces of A.

\subsection{Computing layer spaces from invariant subspaces of $\mathfrak{g}_{f}$}

Algorithm 3 outputs bases of the irreducible invariant subspaces $\left\{\mathcal{V}_{i} \mid i \in[d]\right\}$ of $\mathfrak{g}_{f}$. Recall, we assumed without loss of generality that $\mathcal{V}_{2}$ and $\mathcal{V}_{d-1}$ are subspaces of $\mathcal{V}_{1}$ and $\mathcal{V}_{d}$ respectively. The spaces $\mathcal{V}_{1}, \mathcal{V}_{2}$ and $\mathcal{V}_{d}, \mathcal{V}_{d-1}$ are either the spaces $A^{-1} \mathcal{U}_{1,2}, A^{-1} \mathcal{U}_{2}$ and $A^{-1} \mathcal{U}_{d-1, d}, A^{-1} \mathcal{U}_{d-1}$ respectively, or the spaces $A^{-1} \mathcal{U}_{d-1, d}, A^{-1} \mathcal{U}_{d-1}$ and $A^{-1} \mathcal{U}_{1,2}, A^{-1} \mathcal{U}_{2}$ respectively. Every other $\mathcal{V}_{k}$ is equal to $A^{-1} \mathcal{U}_{\sigma(k)}$ for some permutation $\sigma$ on $[3, d-2](\sigma$ is not known at the end of Algorithm 3). Hence,

$$
\mathbb{F}^{n}=\mathcal{V}_{1} \oplus \mathcal{V}_{3} \oplus \cdots \oplus \mathcal{V}_{d-2} \oplus \mathcal{V}_{d}
$$

\footnotetext{
${ }^{32}$ Identify linear forms with vectors in $\mathbb{F}^{n}$ as mentioned in Definition 15.
} 


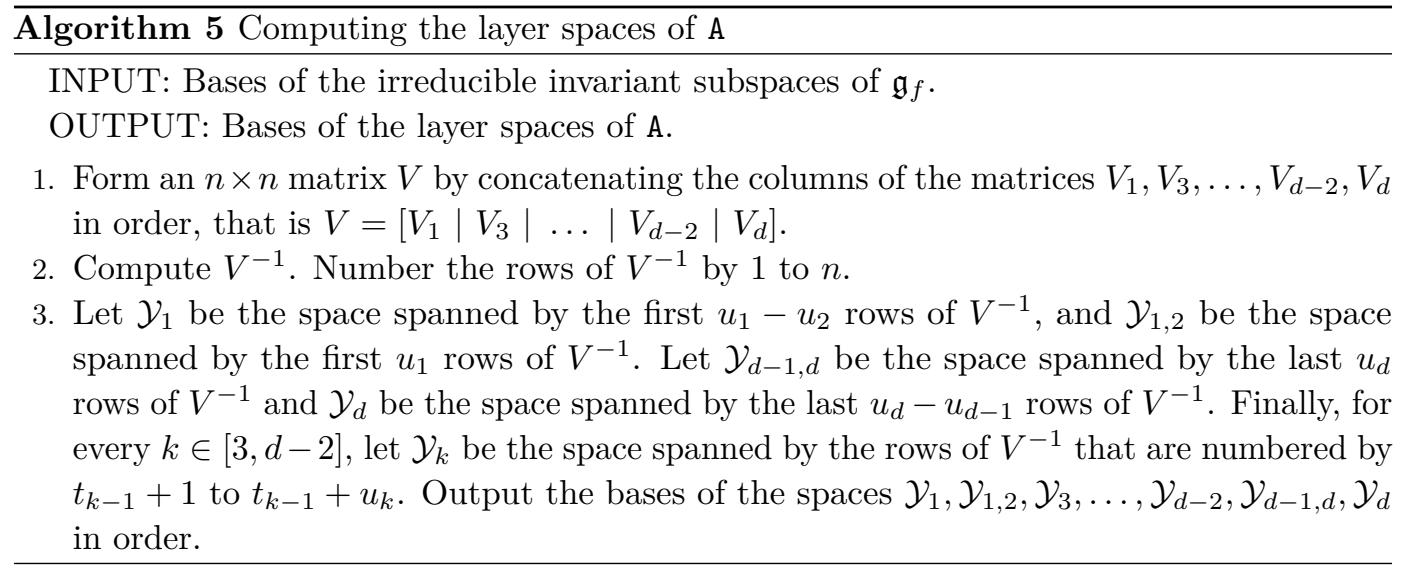

Since $\mathcal{V}_{2} \subseteq \mathcal{V}_{1}$, we can start with a basis of $\mathcal{V}_{2}$ and fill in more elements from the basis of $\mathcal{V}_{1}$ to get a new basis of $\mathcal{V}_{1}$. Thus we can assume the basis of $\mathcal{V}_{2}$ is contained in the basis of $\mathcal{V}_{1}$. Likewise, the basis of $\mathcal{V}_{d-1}$ is contained in the basis of $\mathcal{V}_{d}$.

Order the basis vectors of $\mathcal{V}_{1}$ such that the basis vectors of $\mathcal{V}_{2}$ are at the end and order the basis vectors of $\mathcal{V}_{d}$ such that the basis vectors of $\mathcal{V}_{d-1}$ are at the beginning. For $k \in[3, d-2]$, the basis vectors of $\mathcal{V}_{k}$ are ordered in an arbitrary way. Let $u_{k}$ denote the dimension of $\mathcal{V}_{k}$ for $k \in[d]$. We identify the space $\mathcal{V}_{k}$ with an $n \times u_{k}$ matrix $V_{k}$, where the $i$-th column in $V_{k}$ is the $i$-th basis vector of $\mathcal{V}_{k}$ in the above specified order. Algorithm 5 computes the layer spaces of A using $V_{1}$ to $V_{d}$. Let $t_{2}=u_{1}$ and $t_{k}=u_{k}+t_{k-1}$ for $k \in[3, d-2]$.

Comments on Algorithm 5: Algorithm 2 invokes Algorithm 5 only after Algorithm 3, which returns 'Fail' if $\mathbb{F}^{n} \neq \mathcal{V}_{1} \oplus \mathcal{V}_{3} \oplus \cdots \oplus \mathcal{V}_{d-2} \oplus \mathcal{V}_{d}$ (see comments after Algorithm 3). This ensures Equation (3) is satisfied and so $V^{-1}$ exists in step 2 of the above algorithm, even if there are no $\mathbf{w} \in \mathbb{N}^{d-1}$ and $A \in \mathrm{GL}(n)$ such that $f=\operatorname{IMM}_{\mathbf{w}, d}(A \mathbf{x})$.

- Lemma 49. If $f=X_{1} \cdot X_{2} \cdots X_{d}$ and $\mathcal{Y}_{1}, \mathcal{Y}_{1,2}, \mathcal{Y}_{3}, \ldots, \mathcal{Y}_{d-2}, \mathcal{Y}_{d-1, d}, \mathcal{Y}_{d}$ is the output of Algorithm 5 then there is a permutation $\sigma$ on $[3, d-2]$ such that the following hold:

1. For every $k \in[3, d-2], \mathcal{Y}_{k}=\mathcal{X}_{\sigma(k)}$.

2. Either $\mathcal{Y}_{1}, \mathcal{Y}_{1,2}$ and $\mathcal{Y}_{d}, \mathcal{Y}_{d-1, d}$ are $\mathcal{X}_{1}, \mathcal{X}_{1,2}$ and $\mathcal{X}_{d}, \mathcal{X}_{d-1, d}$ respectively, or $\mathcal{Y}_{1}, \mathcal{Y}_{1,2}$ and $\mathcal{Y}_{d}, \mathcal{Y}_{d-1, d}$ are $\mathcal{X}_{d}, \mathcal{X}_{d-1, d}$ and $\mathcal{X}_{1}, \mathcal{X}_{1,2}$ respectively.

The proof is given in Section 7.4.

\subsection{Reduction to almost set-multilinear ABP}

The outline: Once the invariant spaces of $\mathfrak{g}_{f}$ are computed, the reduction proceeds like this: As observed in the proof of Lemma 49, the matrix $V$ in Algorithm 5 equals $A^{-1} E$ where $E$ looks as shown in Figure 14. If $f=\mathrm{IMM}_{\mathbf{w}, d}(A \mathbf{x})$ then $f(V \mathbf{x})=\mathrm{IMM}_{\mathbf{w}, d}(E \mathbf{x})$. Owing to the structure of $E, f(V \mathbf{x})$ is computed by a full rank almost set-multilinear ABP, except that the ordering of the groups of variables occurring in the different layers of the ABP is unknown as $\sigma$ is unknown. The 'correct' ordering along with a width vector can be retrieved by applying evaluation dimension, thereby completing the reduction. For a slightly neater presentation of the details (and with the intent of proving Lemma 48), we deviate from this strategy a little bit and make use of the layer spaces that have already been computed by Algorithm 5. 


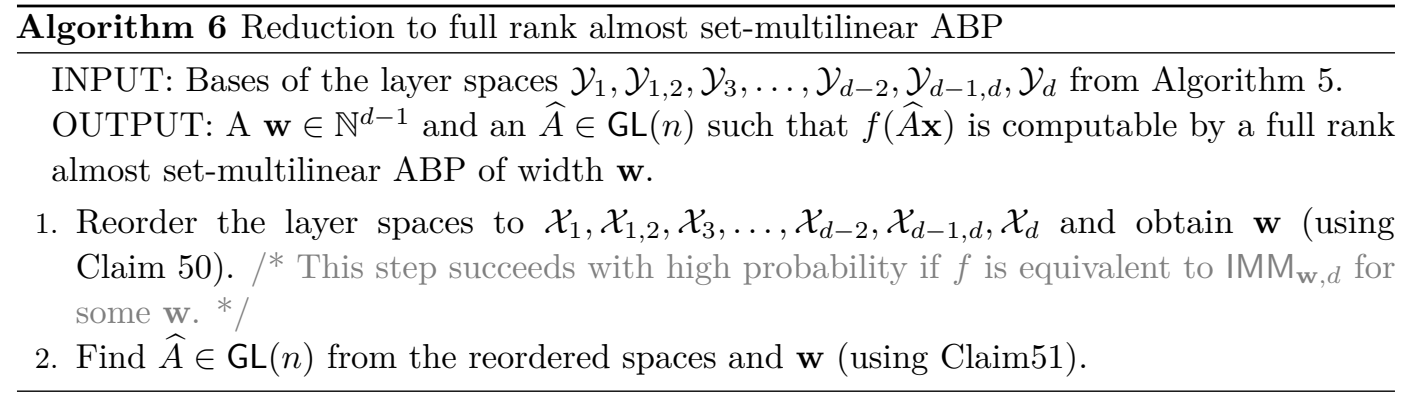

The details: Algorithm 5 computes the spaces $\mathcal{Y}_{1}, \mathcal{Y}_{1,2}, \mathcal{Y}_{3}, \ldots, \mathcal{Y}_{d-2}, \mathcal{Y}_{d-1, d}, \mathcal{Y}_{d}$ which (according to Lemma 49$)$ are either the spaces $\mathcal{X}_{1}, \mathcal{X}_{1,2}, \mathcal{X}_{\sigma(3)}, \ldots, \mathcal{X}_{\sigma(d-2)}, \mathcal{X}_{d-1, d}, \mathcal{X}_{d}$ respectively, or the spaces $\mathcal{X}_{d}, \mathcal{X}_{d-1, d}, \mathcal{X}_{\sigma(3)}, \ldots, \mathcal{X}_{\sigma(d-2)}, \mathcal{X}_{1,2}, \mathcal{X}_{1}$ respectively, for some unknown permutation $\sigma$ on $[3, d-2]$. The claim below (proved in Section 7.4) shows how to correctly reorder these layer spaces.

- Claim 50. There is a randomized polynomial time algorithm that takes input the bases of the layer spaces $\mathcal{Y}_{1}, \mathcal{Y}_{1,2}, \mathcal{Y}_{3}, \ldots, \mathcal{Y}_{d-2}, \mathcal{Y}_{d-1, d}, \mathcal{Y}_{d}$ and with probability at least $1-\frac{1}{\operatorname{poly}(n)}$ reorders these layer spaces and outputs a width vector $\mathbf{w}^{\prime}$ such that the reordered sequence of spaces and $\mathbf{w}^{\prime}$ are:

1. either $\mathcal{X}_{1}, \mathcal{X}_{1,2}, \mathcal{X}_{3}, \ldots, \mathcal{X}_{d-2}, \mathcal{X}_{d-1, d}, \mathcal{X}_{d}$ and $\left(w_{1}, w_{2}, \ldots, w_{d-1}\right)$ respectively,

2. or $\mathcal{X}_{d}, \mathcal{X}_{d-1, d}, \mathcal{X}_{d-2}, \ldots, \mathcal{X}_{3}, \mathcal{X}_{1,2}, \mathcal{X}_{1}$ and $\left(w_{d}, w_{d-1}, \ldots, w_{1}\right)$ respectively.

Note: Until the algorithm in the claim is applied to reorder the spaces, Algorithm 2 is totally oblivious of the width vector $\mathbf{w}$ (it has been used only in the analysis thus far). So, due to the legitimacy of the transposition transformation mentioned at the beginning of this section, we may as well assume that the $\mathbf{w}^{\prime}$ in the above claim is in fact our $\mathbf{w}$, and the output ordered sequence of spaces is $\mathcal{X}_{1}, \mathcal{X}_{1,2}, \mathcal{X}_{3}, \ldots, \mathcal{X}_{d-2}, \mathcal{X}_{d-1, d}, \mathcal{X}_{d}$.

- Claim 51. Given bases of the spaces $\mathcal{X}_{1}, \mathcal{X}_{1,2}, \mathcal{X}_{3}, \ldots, \mathcal{X}_{d-2}, \mathcal{X}_{d-1, d}, \mathcal{X}_{d}$ and $\mathbf{w}$, we can find an $\widehat{A} \in \mathrm{GL}(n)$ in polynomial time such that $f(\widehat{A} \mathbf{x})$ is computable by a full rank almost set-multilinear $A B P$ of width $\mathbf{w}$.

Proof. Identify the variables $x_{1}, \ldots, x_{n}$ with the variables $\mathbf{x}_{1} \uplus \ldots \uplus \mathbf{x}_{d}$ of $\mathrm{IMM}_{\mathbf{w}, d}$ following the ordering prescribed in Section 2.3. The map $\mathbf{x} \mapsto \widehat{A} \mathbf{x}$ should satisfy the following conditions:

(a) For every $k \in[3, d-2]$, the linear forms corresponding ${ }^{33}$ to the basis vectors of $\mathcal{X}_{k}$ map to distinct variables in $\mathbf{x}_{k}$.

(b) The linear forms corresponding to the basis vectors in $\mathcal{X}_{1}$ (similarly, $\mathcal{X}_{d}$ ) map to distinct variables in $\mathbf{x}_{1}$ ( similarly, $\mathbf{x}_{d}$ ).

(c) The linear forms corresponding to the basis vectors in $\mathcal{X}_{1,2}$ (similarly, $\mathcal{X}_{d-1, d}$ ) map to distinct variables in $\mathbf{x}_{1} \uplus \mathbf{x}_{2}$ ( similarly, $\mathbf{x}_{d-1} \uplus \mathbf{x}_{d}$ ).

Conditions (b) and (c) can be simultaneously satisfied as the basis of $\mathcal{X}_{1}$ (similarly, $\mathcal{X}_{d}$ ) is contained in the basis of $\mathcal{X}_{1,2}$ (similarly, $\mathcal{X}_{d-1, d}$ ) by construction. Such an $\widehat{A}$ can be easily obtained.

We summarize the discussion in Algorithm 6.

\footnotetext{
${ }^{33}$ Recall, linear forms in $\mathbf{x}$ variables and vectors in $\mathbb{F}^{n}$ are naturally identified with each other.
} 
Comments on Algorithm 6: The proof of Claim 50 includes Observation 67 which helps Algorithm 6 in step 1 to reorder the layer spaces. If $f$ is not equivalent to $\mathrm{IMM}_{\mathbf{w}, d}$ for some w then Algorithm 6 may fail in step 1, as at some stage it may not be able to find a variable set $\mathbf{z}_{k}$ such that Evaldim $\mathbf{y}_{j} \uplus \mathbf{z}_{k}(h)<\left|\mathbf{z}_{k}\right|$ (see proof of Observation 67). When Algorithm 2 invokes Algorithm 6, if step 1 fails then the latter outputs 'Fail' and stops.

\subsection{Reconstructing almost set-multilinear ABP}

We prove Claim 30 in this section. Let $h=f(\widehat{A} \mathbf{x})$; identify $\mathbf{x}$ with the variables $\mathbf{x}_{1} \uplus \ldots \uplus \mathbf{x}_{d}$ of $\mathrm{IMM}_{\mathbf{w}, d}$ as before. From Claim $51, h$ is computable by a full rank almost set-multilinear ABP of width w. Algorithm 2 uses Algorithm 7 to reconstruct a full rank almost set-multilinear ABP for $h$ and then it replaces $\mathbf{x}$ by $\widehat{A}^{-1} \mathbf{x}$ to output a full rank ABP for $f$. The correctness of Algorithm 7 is presented as part of the proof of Claim 30. We begin with the following two observations the proofs of which appear in Section 7.4.

- Observation 52. If $h$ is computable by a full rank almost set-multilinear ABP of width $\mathbf{w}$ then there is a full rank almost set-multilinear ABP of width $\mathbf{w}$ in canonical form computing $h$.

- Observation 53. Let $X_{1} \cdot X_{2} \cdots X_{d}$ be a full rank almost set-multilinear $A B P$, and $C_{k}=$ $X_{k} \cdots X_{d}$ for $k \in[2, d]$. Let the $l$-th entry of $C_{k}$ be $h_{k l}$ for $l \in\left[w_{k-1}\right]$. Then the polynomials $\left\{h_{k 1}, h_{k 2}, \cdots, h_{k w_{k-1}}\right\}$ are $\mathbb{F}$-linearly independent.

Notations for Algorithm 7: For $k \in[d-1]$, let $t_{k}=\left|\mathbf{x}_{1} \uplus \mathbf{x}_{2} \uplus \cdots \uplus \mathbf{x}_{k}\right|$ and $m_{k}=$ $\left|\mathbf{x}_{k+1} \uplus \mathbf{x}_{k+2} \uplus \cdots \uplus \mathbf{x}_{d}\right|$. The $(i, j)$-th entry of a matrix $X$ is denoted by $X(i, j)$, and $e_{w_{k}, i}$ denotes a vector in $\mathbb{F}^{w_{k}}$ with the $i$-th entry 1 and other entries 0 . Let $\mathbf{y}_{i}$ denote the following partial assignment to the $\mathbf{x}_{1}$ variables: $x_{i}^{(1)}, \ldots, x_{w_{1}}^{(1)}$ are kept intact, while the remaining variables are set to zero. Similarly, $\mathbf{z}_{j}$ denotes the following partial assignment to the $\mathbf{x}_{d}$ variables: $x_{j}^{(d)}, \ldots, x_{w_{d-1}}^{(d)}$ are kept intact, while the remaining variables are set to zero. The notation $h\left(\mathbf{a}_{i}, \mathbf{x}_{k}, \mathbf{b}_{j}\right)$ means the variables $\mathbf{x}_{1} \uplus \ldots \uplus \mathbf{x}_{k-1}$ are given the assignment $\mathbf{a}_{i} \in \mathbb{F}^{t_{k-1}}$ and the variables $\mathbf{x}_{k+1} \uplus \ldots \uplus \mathbf{x}_{d}$ are given the assignment $\mathbf{b}_{j} \in \mathbb{F}^{m_{k}}$. The connotations for $h\left(\mathbf{y}_{i}, \mathbf{x}_{2}, \mathbf{b}_{j}\right)$ and $h\left(\mathbf{a}_{i}, \mathbf{x}_{d-1}, \mathbf{z}_{j}\right)$ are similar. The function $\operatorname{poly}(n)$ is a suitably large polynomial function in $n$, say $n^{7}$.

Proof of Claim 30. By Observation 52, there is a full rank ABP $X_{1}^{\prime} \cdot X_{2}^{\prime} \cdots X_{d}^{\prime}$ in canonical form computing $h$. Hence $X_{1}=X_{1}^{\prime}=\left(x_{1}^{(1)} x_{2}^{(1)} \ldots x_{w_{1}}^{(1)}\right)$ and $X_{d}=X_{d}^{\prime}=\left(x_{1}^{(d)} x_{2}^{(d)} \ldots x_{w_{d-1}}^{(d)}\right)$. We show next that with probability at least $1-\frac{1}{\operatorname{poly}(n)}$, Algorithm 7 constructs $X_{2}, X_{3}, \ldots$, $X_{d-1}$ such that $X_{2}=X_{2}^{\prime} \cdot T_{2}, X_{d-1}=T_{d-2}^{-1} \cdot X_{d-1}^{\prime}$ and $X_{k}=T_{k-1}^{-1} \cdot X_{k}^{\prime} \cdot T_{k}$ for every $k \in[3, d-2]$, where $T_{i} \in \mathrm{GL}\left(w_{i}\right)$ for $i \in[2, d-2]$.

Steps 3-13: The matrix $X_{2}$ is formed in these steps. By Observation 53, the polynomials $h_{31}, \ldots, h_{3 w_{2}}$ are $\mathbb{F}$-linearly independent. Since $\mathbf{b}_{1}, \mathbf{b}_{2}, \ldots, \mathbf{b}_{w_{2}}$ are randomly chosen in step 3 , the matrix $T_{2}$ with $(r, c)$-th entry $h_{3 r}\left(\mathbf{b}_{c}\right)$ is in $\mathrm{GL}\left(w_{2}\right)$ with high probability. Let $X_{2}^{\prime} T_{2}(i, j)$ be the $(i, j)$-th entry of $X_{2}^{\prime} T_{2}$. Observe that

$$
h\left(\mathbf{y}_{i}, \mathbf{x}_{2}, \mathbf{b}_{j}\right)=X_{2}^{\prime} T_{2}(i, j) \cdot x_{i}^{(1)}+\ldots+X_{2}^{\prime} T_{2}\left(w_{1}, j\right) \cdot x_{w_{1}}^{(1)} .
$$

As $h\left(\mathbf{y}_{i}, \mathbf{x}_{2}, \mathbf{b}_{j}\right)$ is a quadratic polynomial, we can compute it from blackbox access using the sparse polynomial interpolation algorithm in [30]. By induction on the rows, $X_{2}(p, j)=$ $X_{2}^{\prime} T_{2}(p, j)$ for every $p \in\left[i+1, w_{1}\right]$ and $j \in\left[w_{2}\right]$. So in step $8, g_{j}=X_{2}^{\prime} T_{2}(i, j) \cdot x_{i}^{(1)}$ leading to $X_{2}(i, j)=X_{2}^{\prime} T_{2}(i, j)$ in step 9 . 


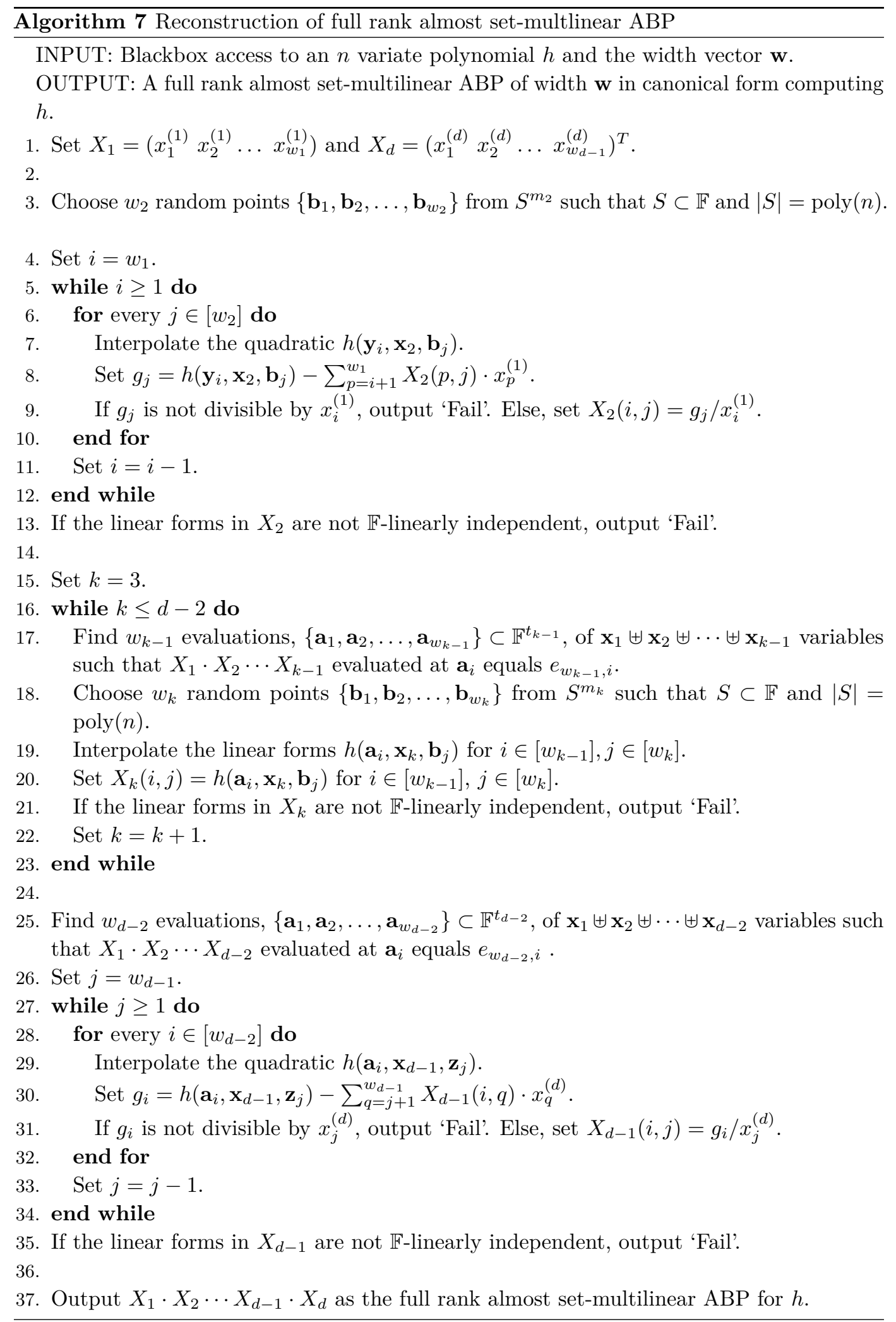


Steps 15-23: The matrices $X_{3}, \ldots, X_{d-2}$ are formed in these steps. By the time the algorithm reaches step 17 , it has already computed $X_{2}, \ldots, X_{k-1}$ such that $X_{2}=X_{2}^{\prime} T_{2}$ and $X_{q}=T_{q-1}^{-1} X_{q}^{\prime} T_{q}$ for $q \in[3, k-1]$, where $T_{q} \in \mathrm{GL}\left(w_{q}\right)$. So, $X_{1}^{\prime} \ldots X_{k-1}^{\prime}=X_{1} \ldots X_{k-1} T_{k-1}^{-1}$. As the linear forms in $X_{1}, \ldots, X_{k-1}$ are $\mathbb{F}$-linearly independent (otherwise the algorithm would have terminated in step 13 or 21 ), we can easily compute points $\left\{\mathbf{a}_{1}, \mathbf{a}_{2}, \ldots, \mathbf{a}_{w_{k-1}}\right\}$ satisfying the required condition in step 17. By Observation 53, the polynomials $h_{(k+1) 1}, \ldots, h_{(k+1) w_{k}}$ are $\mathbb{F}$-linearly independent. Since $\mathbf{b}_{1}, \mathbf{b}_{2}, \ldots, \mathbf{b}_{w_{k}}$ are randomly chosen in step 18 , the matrix $T_{k}$ with $(r, c)$-th entry $h_{(k+1) r}\left(\mathbf{b}_{c}\right)$ is in $\mathrm{GL}\left(w_{k}\right)$ with high probability. Now observe that $h\left(\mathbf{a}_{i}, \mathbf{x}_{k}, \mathbf{b}_{j}\right)$ is the $(i, j)$-th entry of $T_{k-1}^{-1} X_{k}^{\prime} T_{k}$, which implies $X_{k}=T_{k-1}^{-1} X_{k}^{\prime} T_{k}$ from step 20.

Steps 25-35: In these steps, matrix $X_{d-1}$ is formed. The argument showing $X_{d-1}=$ $T_{d-2}^{-1} X_{d-1}^{\prime}$ is similar to the argument used for steps $3-13$, except that now we induct on columns instead of rows.

The output $\mathrm{ABP} X_{1} \ldots X_{d}$ is in canonical form as $X_{1}^{\prime} \ldots X_{d}^{\prime}$ is also in canonical form. It is clear that the total running time of the algorithm is $\operatorname{poly}(n, \beta)$, where $\beta$ is the bit length of the coefficients of $h$ which influences the bit length of the values returned by the blackbox.

\section{Symmetries of IMM}

Recall from Section 2.3, IMM $\mathrm{IM}_{\mathbf{w}, d}$ (for brevity IMM) is the $n$ variate polynomial computed by the full rank ABP $Q_{1} \cdot Q_{2} \cdots Q_{d}$ where the set of variables in $Q_{k}$ is $\mathbf{x}_{k}$ for every $k \in[d]$. In this section, we determine the group of symmetries of IMM (denoted by $\mathcal{G}_{\text {IMM }}$ ) and show that IMM is characterized by its symmetries. We make a note of a few notations and terminologies below.

\section{Notations}

- Calligraphic letters $\mathcal{H}, \mathcal{C}, \mathcal{M}$ and $\mathcal{T}$ denote subgroups of $\mathcal{G}_{\text {Iмм }}$. Let $\mathcal{C}$ and $\mathcal{H}$ be subgroups of $\mathcal{G}_{\text {IMM }}$ such that $\mathcal{C} \cap \mathcal{H}=I_{n}$ and for every $H \in \mathcal{H}$ and $C \in \mathcal{C}, H \cdot C \cdot H^{-1} \in \mathcal{C}$. Then $\mathcal{C} \rtimes \mathcal{H}$ denotes the semidirect product of $\mathcal{C}$ and $\mathcal{H}^{34}$.

- For every $A \in \mathcal{G}_{\text {IMM }}$ the full rank ABP obtained by replacing $\mathbf{x}$ by $A \mathbf{x}$ in $Q_{1} \cdot Q_{2} \cdots Q_{d}$ is termed as the full rank ABP determined by $A$. This full rank ABP also computes IMM.

- Let $X$ be a matrix with entries as linear forms in $\mathbf{y} \uplus \mathbf{z}$ variables. We break $X$ into two parts $X(\mathbf{y})$ and $X(\mathbf{z})$ such that $X=X(\mathbf{y})+X(\mathbf{z})$. The $(i, j)$-th linear form in $X(\mathbf{y})$ (respectively $X(\mathbf{z}))$ is the part of the $(i, j)$-th linear form of $X$ in $\mathbf{y}$ (respectively $\mathbf{z}$ ) variables.

\subsection{The group $\mathcal{G}_{\text {IMM }}$}

Three subgroups of $\mathcal{G}_{\text {IMM }}$ : As before, let $\mathbf{w}=\left(w_{1}, w_{2}, \ldots, w_{d-1}\right)$ and $w_{k}>1$ for every $k \in[d-1]$. In Theorem 54 below, we show that $\mathcal{G}_{\mathrm{IMM}}$ is generated by three special subgroups.

1. Transposition subgroup $\mathcal{T}$ : If $w_{k} \neq w_{d-k}$ for any $k \in[d-1]$ then $\mathcal{T}$ is the trivial group containing only $I_{n}$. Otherwise, if $w_{k}=w_{d-k}$ for every $k \in[d-1]$ then $\mathcal{T}$ is the group consisting of two elements $I_{n}$ and $T$. The matrix $T$ is such that the full rank ABP determined by $T$ is $Q_{d}^{T} \cdot Q_{d-1}^{T} \cdots Q_{1}^{T}$. Clearly, $T$ is a permutation matrix and $T^{2}=I_{n}$.

\footnotetext{
${ }^{34} \mathcal{C} \rtimes \mathcal{H}$ is the set $\mathcal{C H}$ which can be easily shown to be a subgroup of $\mathcal{G}_{\text {IMM }}$, and it also follows that $\mathcal{C}$ is a normal subgroup of $\mathcal{C} \rtimes \mathcal{H}$.
} 
2. Left-right multiplications subgroup $\mathcal{M}$ : $\operatorname{An} M \in \mathrm{GL}(n)$ is in $\mathcal{M}$ if and only if the full rank $\mathrm{ABP} X_{1} \cdot X_{2} \cdots X_{d}$ determined by $M$ has the following structure: There are matrices $A_{1}, \ldots, A_{d-1}$ with $A_{k} \in \mathrm{GL}\left(w_{k}\right)$ for every $k \in[d-1]$, such that $X_{1}=Q_{1} \cdot A_{1}$, $X_{d}=A_{d-1}^{-1} \cdot Q_{d}$, and $X_{k}=A_{k-1}^{-1} \cdot Q_{k} \cdot A_{k}$ for $k \in[2, d-1]$. It is easy to verify that $\mathcal{M}$ is a subgroup of $\mathcal{G}_{\mathrm{IMM}}$ and is isomorphic to the direct product $\mathrm{GL}\left(w_{1}\right) \times \ldots \times \mathrm{GL}\left(w_{d-1}\right)$.

3. Corner translations subgroup $\mathcal{C}: \mathrm{A} C \in \mathrm{GL}(n)$ is in $\mathcal{C}$ if and only if the full rank ABP $X_{1} \cdot X_{2} \cdots X_{d}$ determined by $C$ has the following structure: There are two sets $\left\{C_{11}, C_{12}, \ldots, C_{1 w_{2}}\right\}$ and $\left\{C_{d 1}, C_{d 2}, \ldots, C_{d w_{d-2}}\right\}$ containing anti-symmetric matrices in $\mathbb{F}^{w_{1} \times w_{1}}$ and $\mathbb{F}^{w_{d-1} \times w_{d-1}}$ respectively such that $X_{2}=Q_{2}+Y_{2}$ and $X_{d-1}=Q_{d-1}+Y_{d-1}$, where $Y_{2} \in \mathbb{F}\left[\mathbf{x}_{1}\right]^{w_{1} \times w_{2}}$ (respectively $Y_{d-1} \in \mathbb{F}\left[\mathbf{x}_{d}\right]^{w_{d-2} \times w_{d-1}}$ ) is a matrix with its $i$-th column (respectively $i$-th row) equal to $C_{1 i} \cdot Q_{1}^{T}$ (respectively $Q_{d}^{T} \cdot C_{d i}$ ). For every other $k \in[d] \backslash\{2, d-1\}, X_{k}=Q_{k}$. Observe that $Q_{1} \cdot C_{1 i} \cdot Q_{1}^{T}=Q_{d}^{T} \cdot C_{d i} \cdot Q_{d}=0$. It can also be verified that $\mathcal{C}$ is an abelian subgroup of $\mathcal{G}_{\text {IMM }}$ and is isomorphic to the direct product $\mathcal{A}_{w_{1}}^{w_{2}} \times \mathcal{A}_{w_{d-1}}^{w_{d-2}}$, where $\mathcal{A}_{w}$ is the group of $w \times w$ anti-symmetric matrices under matrix addition and $\mathcal{A}_{w}^{k}$ is the $k$ times direct product of this group.

Theorem 54 (Symmetries of IMM). $\mathcal{G}_{\text {IMM }}=\mathcal{C} \rtimes \mathcal{H}$, where $\mathcal{H}=\mathcal{M} \rtimes \mathcal{T}$.

We prove Theorem 54 below. Following are a couple of remarks on it.

\section{Remarks}

(a) Characterization: Let $f$ be an $n$ variate degree $d$ polynomial satisfying the following: For any $n$ variate degree $d$ polynomial $g, \mathcal{G}_{f}=\mathcal{G}_{g}$ if and only if $f=\alpha \cdot g$ for some nonzero $\alpha \in \mathbb{F}$. Then $f$ is said to be characterized by $\mathcal{G}_{f}$. We prove IMM is characterized by $\mathcal{G}_{\text {IMM }}$ in Lemma 59. The groups $\mathcal{M}$ and $\mathcal{C}$ generate the 'continuous symmetries' of IMM.

(b) Comparison with a related work: In [15] a different choice of the IMM polynomial is considered, namely the trace of a product of $d$ square symbolic matrices - let us call this polynomial $\mathrm{IMM}^{\prime 35}$. The group of symmetries of $\mathrm{IMM}^{\prime}$ is determined in [15] and it is shown that $\mathrm{IMM}^{\prime}$ is characterized by $\mathcal{G}_{\mathrm{IMM}^{\prime}}$. The group of symmetries of $\mathrm{IMM}^{\prime}$, like IMM, is generated by the transposition subgroup, the left-right multiplication subgroup, and (instead of the corner translations subgroup) the circular transformations subgroup - an element in this subgroup cyclically rotates the order of the matrices and hence does not change the trace of the product.

\section{Proof of Theorem 54}

We begin with the following observation which is immediate from Lemma 48.

- Observation 55. If $X_{1} \cdot X_{2} \cdots X_{d}$ is a width $\mathbf{w}^{\prime}=\left(w_{1}^{\prime}, w_{2}^{\prime}, \ldots, w_{d-1}^{\prime}\right)$ full rank $A B P$ computing $\mathrm{IMM}_{\mathbf{w}, d}$ then either

1. $w_{k}^{\prime}=w_{k}$ for $k \in[d-1]$, and the spaces $\mathcal{X}_{1}, \mathcal{X}_{1,2}, \mathcal{X}_{3}, \ldots, \mathcal{X}_{d-1, d}, \mathcal{X}_{d}$ are the spaces $\mathcal{Q}_{1}, \mathcal{Q}_{1,2}, \mathcal{Q}_{3}, \ldots, \mathcal{Q}_{d-1, d}, \mathcal{Q}_{d}$ respectively, or

2. $w_{k}^{\prime}=w_{d-k}$ for $k \in[d-1]$, and the spaces $\mathcal{X}_{1}, \mathcal{X}_{1,2}, \mathcal{X}_{3}, \ldots, \mathcal{X}_{d-1, d}, \mathcal{X}_{d}$ are the spaces $\mathcal{Q}_{d}, \mathcal{Q}_{d-1, d}, \mathcal{Q}_{d-2}, \ldots, \mathcal{Q}_{1,2}, \mathcal{Q}_{1}$ respectively.

\footnotetext{
${ }^{35}$ The complexities of IMM and $\mathrm{IMM}^{\prime}$ are polynomially related to each other, in particular both are complete for algebraic branching programs under p-projections. But their groups of symmetries are slightly different.
} 


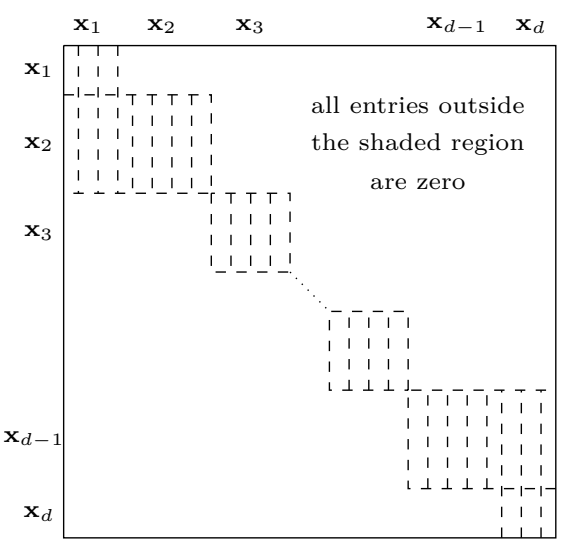

Figure 5 Matrix $A$ in $\mathcal{G}_{\mathrm{IMM}}$.

From the definitions of $\mathcal{T}, \mathcal{M}$ and $\mathcal{C}$ it follows that $\mathcal{C} \cap \mathcal{M}=\mathcal{C} \cap \mathcal{T}=\mathcal{M} \cap \mathcal{T}=I_{n}$. The claim below shows $\mathcal{G}_{\text {Iмм }}$ is generated by $\mathcal{C}, \mathcal{M}$ and $\mathcal{T}$.

- Claim 56. For every $A \in \mathcal{G}_{\mathrm{IMM}}$, there exist $C \in \mathcal{C}, M \in \mathcal{M}$ and $\tilde{T} \in \mathcal{T}$ such that $A=C \cdot M \cdot \tilde{T}$.

Proof. Let $X_{1} \cdot X_{2} \cdots X_{d}$ be the full rank ABP A of width w determined by $A$. If $w_{k}=w_{d-k}$ for $k \in[d-1]$ then the spaces $\mathcal{X}_{1}, \mathcal{X}_{1,2}, \mathcal{X}_{3}, \ldots, \mathcal{X}_{d-1, d}, \mathcal{X}_{d}$ are either equal to $\mathcal{Q}_{1}, \mathcal{Q}_{1,2}, \mathcal{Q}_{3}$, $\ldots, \mathcal{Q}_{d-1, d}, \mathcal{Q}_{d}$ respectively or $\mathcal{Q}_{d}, \mathcal{Q}_{d-1, d}, \mathcal{Q}_{d-2}, \ldots, \mathcal{Q}_{1,2}, \mathcal{Q}_{1}$ respectively (from Observation 55). Otherwise if $w_{k} \neq w_{d-k}$ for any $k \in[d-1]$ then the spaces $\mathcal{X}_{1}, \mathcal{X}_{1,2}, \mathcal{X}_{3}, \ldots$, $\mathcal{X}_{d-1, d}, \mathcal{X}_{d}$ have only one choice and are equal to $\mathcal{Q}_{1}, \mathcal{Q}_{1,2}, \mathcal{Q}_{3}, \ldots, \mathcal{Q}_{d-1, d}, \mathcal{Q}_{d}$ respectively. We deal with these two choices of layer spaces separately.

Case A: $\quad$ Suppose $\mathcal{X}_{1}, \mathcal{X}_{1,2}, \mathcal{X}_{3}, \ldots, \mathcal{X}_{d-1, d}, \mathcal{X}_{d}$ are equal to $\mathcal{Q}_{1}, \mathcal{Q}_{1,2}, \mathcal{Q}_{3}, \ldots, \mathcal{Q}_{d-1, d}, \mathcal{Q}_{d}$ respectively. Hence $A$ looks as shown in Figure 5.

The linear forms in $X_{2}, X_{d-1}$ are in variables $\mathbf{x}_{1} \uplus \mathbf{x}_{2}, \mathbf{x}_{d-1} \uplus \mathbf{x}_{d}$ respectively. Further,

$$
\prod_{k=1}^{d} X_{k}=X_{1} \cdot\left(X_{2}\left(\mathbf{x}_{1}\right)+X_{2}\left(\mathbf{x}_{2}\right)\right) \cdot\left(\prod_{k=3}^{d-2} X_{k}\right) \cdot\left(X_{d-1}\left(\mathbf{x}_{d-1}\right)+X_{d-1}\left(\mathbf{x}_{d}\right)\right) \cdot X_{d}=\text { IMM. }^{36}
$$

Since $\mathrm{A}$ is a full rank $\mathrm{ABP}$ and each monomial in IMM contains one variable from each set $\mathbf{x}_{k}$

$$
X_{1} \cdot X_{2}\left(\mathbf{x}_{2}\right) \cdot\left(\prod_{k=3}^{d-2} X_{k}\right) \cdot X_{d-1}\left(\mathbf{x}_{d-1}\right) \cdot X_{d}=\text { IMM, and also }
$$

$X_{1} \cdot X_{2}\left(\mathbf{x}_{1}\right) \cdot \prod_{k=3}^{d-2} X_{k} \cdot X_{d-1}\left(\mathbf{x}_{d-1}\right) \cdot X_{d}=0 \quad$ and $\quad X_{1} \cdot X_{2}\left(\mathbf{x}_{2}\right) \cdot \prod_{k=3}^{d-2} X_{k} \cdot X_{d-1}\left(\mathbf{x}_{d}\right) \cdot X_{d}=0$ implying

$$
X_{1} \cdot X_{2}\left(\mathbf{x}_{1}\right)=0_{w_{2}}^{T} \text { and } \quad X_{d-1}\left(\mathbf{x}_{d}\right) \cdot X_{d}=0_{w_{d-2}}
$$

where $0_{w}$ is a zero (column) vector in $\mathbb{F}^{w}$. Observation 57 , the proof of which is in Section 7.5, proves the existence of a matrix $M \in \mathcal{M}$ such that the full rank ABP determined by $M$ is $X_{1} \cdot X_{2}\left(\mathbf{x}_{2}\right) \cdot X_{3} \cdots X_{d-2} \cdot X_{d-1}\left(\mathbf{x}_{d-1}\right) \cdot X_{d}$. 
- Observation 57. There are matrices $A_{1}, \ldots, A_{d-1}$ with $A_{k} \in \mathrm{GL}\left(w_{k}\right)$ for every $k \in[d-1]$, such that $X_{1}=Q_{1} \cdot A_{1}, X_{2}\left(\mathbf{x}_{2}\right)=A_{1}^{-1} \cdot Q_{2} \cdot A_{2}, X_{d-1}\left(\mathbf{x}_{d-1}\right)=A_{d-2}^{-1} \cdot Q_{d-1} \cdot A_{d-1}$, $X_{d}=A_{d-1}^{-1} \cdot Q_{d}$, and $X_{k}=A_{k-1}^{-1} \cdot Q_{k} \cdot A_{k}$ for $k \in[3, d-2]$.

We now show the existence of a $C \in \mathcal{C}$ such that the full rank $\mathrm{ABP}$ determined by $C \cdot M$ is $X_{1} \cdot X_{2} \cdots X_{d}$, from which the claim follows by letting $\tilde{T}=I_{n}$. Since the linear forms in $X_{1}$ are $\mathbb{F}$-linearly independent, there are $w_{1} \times w_{1}$ matrices $\left\{C_{11}, C_{12}, \ldots, C_{1 w_{2}}\right\}$ such that the $i$-th column of $X_{2}\left(\mathbf{x}_{1}\right)$ is $C_{1 i} X_{1}^{T}$. So from Equation (4), $X_{1} \cdot C_{1 i} \cdot X_{1}^{T}=0$ (equivalently $\left.Q_{1} \cdot C_{1 i} \cdot Q_{1}^{T}=0\right)$ implying $C_{1 i}$ is an anti-symmetric matrix for every $i \in\left[w_{2}\right]$. Similarly, there are $w_{d-1} \times w_{d-1}$ anti-symmetric matrices $\left\{C_{d 1}, C_{d 2}, \ldots, C_{d w_{d-2}}\right\}$ such that the $i$-th row of $X_{d-1}\left(\mathbf{x}_{d}\right)$ is $X_{d}^{T} C_{d i}$. Let $C \in \mathrm{GL}(n)$ be such that the ABP determined by it is $Q_{1} Q_{2}^{\prime} Q_{3} \cdots Q_{d-2} Q_{d-1}^{\prime} Q_{d}$ where $Q_{2}^{\prime}=Q_{2}+Y_{2}$ and $Q_{d-1}^{\prime}=Q_{d-1}+Y_{d-1}$, the $i$-th column (respectively $i$-th row) of $Y_{2}$ (respectively $Y_{d-1}$ ) is $C_{1 i} Q_{1}^{T}$ (respectively $Q_{d-1}^{T} C_{d i}$ ). By construction, $C \in \mathcal{C}$ and the ABP determined by $C \cdot M$ is $X_{1} \cdot X_{2} \cdots X_{d}$.

Case B: $\quad$ Suppose $\mathcal{X}_{1}, \mathcal{X}_{1,2}, \mathcal{X}_{3}, \ldots, \mathcal{X}_{d-1, d}, \mathcal{X}_{d}$ are the spaces $\mathcal{Q}_{d}, \mathcal{Q}_{d-1, d}, \mathcal{Q}_{d-2}, \ldots, \mathcal{Q}_{1,2}, \mathcal{Q}_{1}$ respectively. This implies $w_{k}=w_{d-k}$ for $k \in[d-1]$ and hence the full rank ABP determined by $T$ is $Q_{d}^{T} \cdot Q_{d-1}^{T} \cdots Q_{1}^{T}$. From here the existence of $M \in \mathcal{M}$ and $C \in \mathcal{C}$ such that the full rank ABP determined by $M \cdot C \cdot T$ is $X_{1} \cdot X_{2} \cdots X_{d}$ follows just as in Case A. This completes the proof of the claim.

Observe that if $T \in \mathcal{T}$ then for every $M \in \mathcal{M}, T \cdot M \cdot T^{-1} \in \mathcal{M}$. Let $\mathcal{H}=\mathcal{M} \rtimes \mathcal{T}$. Clearly, $\mathcal{C} \cap \mathcal{H}=I_{n}$. The following claim along with Claim 56 then conclude the proof of Theorem 54 .

- Claim 58. For every $C \in \mathcal{C}$ and $H \in \mathcal{H}, H \cdot C \cdot H^{-1} \in \mathcal{C}$.

Proof. Let $H=M \cdot T$ where $M \in \mathcal{M}$ and $T \in \mathcal{T}$, and $A=M T \cdot C \cdot T^{-1} M^{-1}$. Suppose $X_{1} \cdot X_{2} \cdots X_{d-1} \cdot X_{d}$ is the ABP determined by $A$. The matrices $T$ and $T^{-1}$ in $A$ together ensure that the spaces $\mathcal{X}_{1}, \mathcal{X}_{1,2}, \mathcal{X}_{3}, \ldots, \mathcal{X}_{d-1, d}, \mathcal{X}_{d}$ are equal to $\mathcal{Q}_{1}, \mathcal{Q}_{1,2}, \mathcal{Q}_{3}, \ldots, \mathcal{Q}_{d-1, d}, \mathcal{Q}_{d}$ respectively. Also the matrices $M$ and $M^{-1}$ together ensure that $X_{i}=Q_{i}$ for $i \in[d] \backslash\{2, d-1\}$, $X_{2}\left(\mathbf{x}_{2}\right)=Q_{2}$ and $X_{d-1}\left(\mathbf{x}_{d-1}\right)=Q_{d-1}$. Arguing as in Claim 56, we can infer that $A \in \mathcal{C}$.

\subsection{Characterization of IMM by $\mathcal{G}_{\text {IMM }} 0$}

For every $f=\alpha \cdot$ IMM, where $\alpha \in \mathbb{F}$ and $\alpha \neq 0$, it is easily observed that $\mathcal{G}_{f}=\mathcal{G}_{\text {IMм }}$. We prove the converse in the following lemma for any homogeneous degree $d$ polynomial in the $\mathbf{x}$ variables.

- Lemma 59. Let $f$ be a homogeneous degree $d$ polynomial in $n$ variables $\mathbf{x}=\mathbf{x}_{1} \uplus \ldots \uplus \mathbf{x}_{d}$. If $|\mathbb{F}|>d+1$ and the left-right multiplications subgroup $\mathcal{M}$ of $\mathcal{G}_{\mathrm{IMM}}$ is contained in $\mathcal{G}_{f}$ then $f=\alpha \cdot \mathrm{IMM}$ for some nonzero $\alpha \in \mathbb{F}$.

Proof. First, we show that such an $f$ is set-multilinear in the sets $\mathbf{x}_{1}, \ldots, \mathbf{x}_{d}$ : Every monomial in $f$ has exactly one variable from each of the sets $\mathbf{x}_{1}, \ldots, \mathbf{x}_{d}$. As $|\mathbb{F}|>d+1$, there is a nonzero $\rho \in \mathbb{F}$ that is not an $e$-th root of unity for any $e \leq d$. Every element in $\mathcal{M}$ is defined by $d-1$ matrices $A_{1}, \ldots, A_{d-1}$ such that $A_{k} \in \mathrm{GL}\left(w_{k}\right)$ for every $k \in[d-1]$. For a $k \in[d-1]$, consider the element $M \in \mathcal{M}$ that is defined by $A_{k}=\rho \cdot I_{w_{k}}$ and $A_{l}=I_{w_{l}}$ for $l \in[d-1]$ and $l \neq k$. Then, $f(M \cdot \mathbf{x})=f\left(\mathbf{x}_{1}, \ldots, \rho \mathbf{x}_{k}, \rho^{-1} \mathbf{x}_{k+1}, \ldots, \mathbf{x}_{d}\right)$, which by assumption is $f$. Comparing the coefficients of the monomials of $f(M \cdot \mathbf{x})$ and $f$, we observe that in every monomial of $f$ the number of variables from $\mathbf{x}_{k}$ and $\mathbf{x}_{k+1}$ must be the same as $\rho$ is not an $e$-th root of unity for any $e \leq d$. Since $k$ is chosen arbitrarily and $f$ is homogeneous of degree $d, f$ must be set-multilinear in the sets $\mathbf{x}_{1}, \ldots, \mathbf{x}_{d}$. 
The proof is by induction on the degree of $f$. For $i \in\left[w_{1}\right]$, let $\mathbf{x}_{2 i}$ be the set of variables in the $i$-th row of $Q_{2}$, and $Q_{2 i}$ be the $1 \times w_{2}$ matrix containing the $i$-th row of $Q_{2}$. Let $\mathrm{IMM}_{i}$ be the degree $d-1$ iterated matrix multiplication polynomial computed by the ABP $Q_{2 i} \cdot Q_{3} \cdots Q_{d}$. As $f$ is set-multilinear, it can be expressed as

$$
f=g_{1} \cdot x_{1}^{(1)}+\ldots+g_{w_{1}} \cdot x_{w_{1}}^{(1)},
$$

where $g_{1}, \ldots, g_{w_{1}}$ are set-multilinear polynomials in the sets $\mathbf{x}_{2}, \ldots, \mathbf{x}_{d}$. Moreover, we can argue that $g_{i}$ is set-multilinear in $\mathbf{x}_{2 i}, \mathbf{x}_{3}, \ldots, \mathbf{x}_{d}$ as follows: Consider an $N \in \mathcal{M}$ that is defined by a diagonal matrix $A_{1} \in \mathrm{GL}\left(w_{1}\right)$ whose $(i, i)$-th entry is $\rho$ and all other diagonal entries are 1; every other $A_{l}=I_{w_{l}}$ for $l \in[2, d-1]$. The transformation $N$ scales the variable $x_{i}^{(1)}$ by $\rho$ and the variables in $\mathbf{x}_{2 i}$ by $\rho^{-1}$. By comparing the coefficients of the monomials of $f(N \cdot \mathbf{x})$ and $f$, we can conclude that $g_{i}$ is set-multilinear in $\mathbf{x}_{2 i}, \mathbf{x}_{3}, \ldots, \mathbf{x}_{d}$ for every $i \in\left[w_{1}\right]$.

Let $\mathcal{M}^{\prime}$ be the subgroup of $\mathcal{M}$ containing those $M \in \mathcal{M}$ for which $A_{1}=I_{w_{1}}$. From Equation (5), we can infer that $g_{i}(M \cdot \mathbf{x})=g_{i}$ for $M \in \mathcal{M}^{\prime}$, and hence the left-right multiplications subgroup of $\mathcal{G}_{\mathrm{IMM}_{i}}$ is contained in the group of symmetries of $g_{i}$. As degree of $g_{i}$ is $d-1$, by induction ${ }^{37} g_{i}=\alpha_{i} \cdot \mathrm{IMM}_{i}$ for some nonzero $\alpha_{i} \in \mathbb{F}$ and

$$
f=\alpha_{1} \cdot \mathrm{IMM}_{1} \cdot x_{1}^{(1)}+\ldots+\alpha_{w_{1}} \cdot \mathrm{IMM}_{w_{1}} \cdot x_{w_{1}}^{(1)} \cdot
$$

Next we show that $\alpha_{1}=\ldots=\alpha_{w_{1}}$ thereby completing the proof.

For an $i \in\left[2, w_{1}\right]$, let $A_{1} \in \mathrm{GL}\left(w_{1}\right)$ be the upper triangular matrix whose diagonal entries are 1 , the $(1, i)$-th entry is 1 and remaining entries are zero. Let $U$ be the matrix in $\mathcal{M}$ defined by $A_{1}$ and $A_{l}=I_{w_{l}}$ for $l \in[2, d-1]$. The transformation $U$ has the following effect on the variables:

$$
\begin{aligned}
x_{i}^{(1)} & \mapsto x_{1}^{(1)}+x_{i}^{(1)} \text { and } \\
x_{1 j}^{(2)} & \mapsto x_{1 j}^{(2)}-x_{i j}^{(2)} \text { for every } j \in\left[w_{2}\right],
\end{aligned}
$$

every other $\mathbf{x}$ variable maps to itself. Applying $U$ to $f$ in Equation (6) we get

$$
\begin{aligned}
f= & f(U \cdot \mathbf{x})=\alpha_{1} \cdot\left(\mathrm{IMM}_{1}-\mathrm{IMM}_{i}\right) \cdot x_{1}^{(1)}+\ldots+\alpha_{i} \cdot \mathrm{IMM}_{i} \cdot\left(x_{1}^{(1)}+x_{i}^{(1)}\right)+\ldots+ \\
& \alpha_{w_{1}} \cdot \mathrm{IMM}_{w_{1}} \cdot x_{w_{1}}^{(1)} \\
= & f+\left(\alpha_{i}-\alpha_{1}\right) \cdot \mathrm{IMM}_{i} \cdot x_{1}^{(1)} \\
\Rightarrow & \alpha_{i}-\alpha_{1}=0 .
\end{aligned}
$$

Since this is true for any $i \in\left[2, w_{1}\right]$, we have $\alpha_{1}=\ldots=\alpha_{w_{1}}$.

\section{Proof of claims and lemmas from previous sections}

In this section we give proofs of claims and lemmas from the above sections. We begin by proving the incompleteness of the full rank ABP.

- Observation 60. For every sufficiently large $m \in \mathbb{N}$ there is an $m$ variate multilinear polynomial that is not computable by full rank $A B P$.

Proof. A full rank ABP computing an $m$ variate polynomial $f$ has both its width and length bounded by $m$, so $f$ can also be computed by an ABP (not full rank) of width and length exactly $m$. Hence, it is sufficient to show that there is an $m$ variate multilinear polynomial

\footnotetext{
${ }^{37}$ The base case $d=1$ is trivial to show.
} 
that is not computable by the latter kind of ABP. The number of edges in an ABP of width $m$ and length $m$ is $n=m^{2}(m-2)+2 m$. Let these $n$ edges be $e_{1}, e_{2}, \ldots, e_{n}$ and suppose the edge $e_{i}$ is labelled by the affine form $l_{i}=\sum_{j=1}^{m} c_{i j} x_{j}+c_{i 0}$. Treat $c_{i j}$ 's as formal variables. Then each of the $\left(\begin{array}{c}2 n \\ n\end{array}\right)$ coefficients of the polynomial $f$ computed by such an ABP is a polynomial in these $n(m+1)$ formal variables. Since $n(m+1)<2^{m}$ for sufficiently large $m$, the coefficients of $f$ restricted to just the multilinear monomials $\mathfrak{m}_{1}, \mathfrak{m}_{2}, \ldots, \mathfrak{m}_{2^{m}}$ are algebraically dependent. Let $h \neq 0$ be an annihilating polynomial of these coefficients. Since $h$ is nonzero, there is a point $\mathbf{a}=\left(a_{1}, \ldots, a_{2^{m}}\right) \in \mathbb{F}^{2^{m}}$ such that $h(\mathbf{a}) \neq 0$. It follows that the multilinear polynomial $g \stackrel{\text { def }}{=} \sum_{i=1}^{2^{m}} a_{i} \mathfrak{m}_{i}$ is not computable by an ABP of width $m$ and length $m$, which means $g$ is not computable by a full rank ABP.

\subsection{Proof of lemmas and claims in Section 2}

- Claim 21 (restated). If $f(\mathbf{x})=g(A \mathbf{x})$, where $f$ and $g$ are both $n$ variate polynomials and $A \in \mathrm{GL}(n)$, then the Lie algebra of $f$ is a conjugate of the Lie algebra of $g$ via $A$, i.e. $\mathfrak{g}_{f}=\left\{A^{-1} E A: E \in \mathfrak{g}_{g}\right\}=: A^{-1} \mathfrak{g}_{g} A$.

Proof. Let $Q=\left(q_{i, j}\right)_{i, j \in[n]} \in \mathfrak{g}_{f}$. Hence,

$$
\sum_{i, j \in[n]} q_{i j} x_{j} \cdot \frac{\partial f}{\partial x_{i}}=0 \Rightarrow \sum_{i, j \in[n]} q_{i j} x_{j} \cdot \frac{\partial g(A \mathbf{x})}{\partial x_{i}}=0 .
$$

Let $A=\left(a_{k i}\right)_{k, i \in[n]}$. Using chain rule of derivatives,

$$
\frac{\partial g(A \mathbf{x})}{\partial x_{i}}=\sum_{k \in[n]} \frac{\partial g}{\partial x_{k}}(A \mathbf{x}) \cdot a_{k i}
$$

Let $A^{-1}=\left(b_{j l}\right)_{j, l \in[n]}$ and $(A \mathbf{x})_{l}$ be the $l$-th entry of $A \mathbf{x}$. Then $x_{j}=\sum_{l \in[n]} b_{j l}(A \mathbf{x})_{l}$. From Equation (7),

$$
\begin{aligned}
\sum_{i, j \in[n]} q_{i j} \cdot\left(\sum_{l \in[n]} b_{j l}(A \mathbf{x})_{l}\right) \cdot\left(\sum_{k \in[n]} \frac{\partial g}{\partial x_{k}}(A \mathbf{x}) \cdot a_{k i}\right) & =0 \\
\Rightarrow \sum_{k, l \in[n]}(A \mathbf{x})_{l} \cdot \frac{\partial g}{\partial x_{k}}(A \mathbf{x}) \cdot\left(\sum_{i, j \in[n]} a_{k i} q_{i j} b_{j l}\right) & =0, \\
\Rightarrow \sum_{k, l \in[n]} x_{l} \cdot \frac{\partial g}{\partial x_{k}} \cdot\left(\sum_{i, j \in[n]} a_{k i} q_{i j} b_{j l}\right) & \left.=0 \quad \text { (Substituting } \mathbf{x} \text { by } A^{-1} \mathbf{x}\right) .
\end{aligned}
$$

Observe that $\sum_{i, j \in[n]} a_{k i} q_{i j} b_{j l}$ is the $(k, l)$-th entry of $A Q A^{-1}$. Hence, $A Q A^{-1} \in \mathfrak{g}_{g}$ implying $\mathfrak{g}_{f} \subseteq A^{-1} \mathfrak{g}_{g} A$. Similarly, $\mathfrak{g}_{g} \subseteq A \mathfrak{g}_{f} A^{-1}$ as $g=f\left(A^{-1} \mathbf{x}\right)$, implying $\mathfrak{g}_{f}=A^{-1} \mathfrak{g}_{g} A$.

- Claim 24 (restated). With probability at least $1-\frac{1}{\operatorname{poly}(n)}$, the rank of the matrix $M=\left(f_{j}\left(\mathbf{b}_{i}\right)\right)$ where $i, j \in[m]$, is $m-r$ where $\mathbf{b}_{1}, \mathbf{b}_{2}, \ldots, \mathbf{b}_{m}$ are chosen independently and uniformly at random from $S^{n} \subset \mathbb{F}^{n}$ with $|S|=d m \cdot \operatorname{poly}(n)$.

Proof. Recall, we assumed that the dimension of the $\mathbb{F}$-linear space spanned by the $n$ variate polynomials $f_{1}, f_{2}, \ldots, f_{m}$ is $m-r$. Without loss of generality assume $f_{1}, f_{2}, \ldots, f_{m-r}$ form a basis of this linear space. Clearly, the rank of $M=\left(f_{j}\left(\mathbf{b}_{i}\right)\right)_{i, j \in[m]}$ is less than or equal to $m-r$. Let $M_{m-r}=\left(f_{j}\left(\mathbf{b}_{i}\right)\right)_{i, j \in[m-r]}$. That $\operatorname{Det}\left(M_{m-r}\right) \neq 0$ with probability at least $1-\frac{1}{\operatorname{poly}(n)}$ over 
the random choices of $\mathbf{b}_{1}, \mathbf{b}_{2}, \ldots, \mathbf{b}_{m}$ can be argued as follows: Let $\mathbf{y}_{i}=\left\{y_{1}^{(i)}, y_{2}^{(i)}, \ldots, y_{n}^{(i)}\right\}$ for $i \in[m-r]$ be disjoint sets of variables. Rename the $\mathbf{x}=\left\{x_{1}, x_{2}, \ldots, x_{n}\right\}$ variables in $f_{j}(\mathbf{x})$ to $\mathbf{y}_{i}$ and call these new polynomials $f_{j}\left(\mathbf{y}_{i}\right)$ for $i, j \in[m-r]$. Let $Y$ be an $(m-r) \times(m-r)$ matrix whose $(i, j)$-th entry is $\left(f_{j}\left(\mathbf{y}_{i}\right)\right)_{i \in[m-r]}$. Since $f_{1}, f_{2}, \ldots, f_{m-r}$ are $\mathbb{F}$-linearly independent, $\operatorname{Det}(Y) \neq 0$ - this can be argued easily using induction. As $\operatorname{deg}(\operatorname{Det}(Y))=d(m-r) \leq d m$, by Schwartz-Zippel lemma, $\operatorname{Det}\left(M_{m-r}\right) \neq 0$ with probability at least $1-\frac{1}{\operatorname{poly}(n)}$.

- Claim 26 (restated). Let $r$ be the number of redundant variables in an $n$ variate polynomial $f$ of degree $d$. Then the dimension of the space $\mathcal{U}$ of $\mathbb{F}$-linear dependencies of $\left\{\partial_{x_{i}} f \mid i \in[n]\right\}$ is $r$. Moreover, we can construct an $A \in \mathrm{GL}(n)$ in randomized $\operatorname{poly}(n, d, \beta)$ time such that $f(A \mathbf{x})$ is free of the set of variables $\left\{x_{n-r+1}, x_{n-r+2}, \ldots, x_{n}\right\}$ with high probability, where $\beta$ is the bit length of the coefficients of $f$.

Proof. Let $B=\left(b_{i j}\right)_{i, j \in[n]} \in \mathrm{GL}(n)$ such that $f(B \mathbf{x})$ is a polynomial in $x_{1}, x_{2}, \ldots, x_{s}$, where $s=n-r$. For $n-r+1 \leq j \leq n$

$$
\begin{aligned}
\frac{\partial f(B \mathbf{x})}{\partial x_{j}} & =0 \\
\Rightarrow \sum_{i=1}^{n} b_{i j} \cdot \frac{\partial f}{\partial x_{i}}(B \mathbf{x}) & =0 \quad \text { (by chain rule) } \\
\Rightarrow \sum_{i=1}^{n} b_{i j} \cdot \frac{\partial f}{\partial x_{i}} & \left.=0 \quad \text { (substituting } \mathbf{x} \text { by } B^{-1} \mathbf{x}\right) .
\end{aligned}
$$

Since $B \in \mathrm{GL}(n)$, we conclude $\operatorname{dim}(\mathcal{U}) \geq r$. Let $\left\{\left(a_{1 j} a_{2 j} \ldots a_{n j}\right)^{T}:(n-\operatorname{dim}(\mathcal{U})+1) \leq j \leq n\right\}$ be a basis of $\mathcal{U}$. Then,

$$
\sum_{i=1}^{n} a_{i j} \cdot \frac{\partial f}{\partial x_{i}}=0
$$

Let $A \in \mathrm{GL}(n)$ such that for $(n-\operatorname{dim}(\mathcal{U})+1) \leq j \leq n$, the $j$-th column of $A$ is $\left(a_{1 j} a_{2 j} \ldots\right.$ $\left.a_{n j}\right)^{T}$ and the remaining columns of $A$ are arbitrary vectors that make $A$ a full rank matrix. Then,

$$
\sum_{i=1}^{n} a_{i j} \cdot \frac{\partial f}{\partial x_{i}}=0 \Rightarrow \sum_{i=1}^{n} a_{i j} \cdot \frac{\partial f}{\partial x_{i}}(A \mathbf{x})=0 \quad \Rightarrow \quad \frac{\partial f(A \mathbf{x})}{\partial x_{j}}=0 .
$$

This implies $f(A \mathbf{x})$ is a polynomial free of $x_{j}$ variable for $(n-\operatorname{dim}(\mathcal{U})+1) \leq j \leq n$. Hence, $\operatorname{dim}(\mathcal{U}) \leq r$.

Blackbox for polynomials $\partial_{x_{1}} f, \partial_{x_{2}} f, \ldots, \partial_{x_{n}} f$ can be constructed in poly $(n, d, \beta)$ time from blackbox access to $f$ and a basis for the space $\mathcal{U}$ of $\mathbb{F}$-linear dependencies of polynomials $\partial_{x_{1}} f, \partial_{x_{2}} f, \ldots, \partial_{x_{n}} f$ can also be constructed in randomized poly $(n, d, \beta)$ time (see Section 2.2). Thus, we can construct an $A \in \mathrm{GL}(n)$ (similar to the construction shown above) from a blackbox access to $f$ in randomized poly $(n, d, \beta)$ time such that $f(A \mathbf{x})$ is free of the set of variables $\left\{x_{n-r+1}, x_{n-r+2}, \ldots, x_{n}\right\}$. We summarize this in Algorithm 8.

- Lemma 27 (restated). There is a randomized algorithm that takes input blackbox access to two $n$ variate, degree $d$ polynomials $f$ and $g$, and with probability at least $1-\frac{1}{\text { poly }(n)}$ does the following: if $f$ is translation equivalent to $g$, outputs an $\mathbf{a} \in \mathbb{F}^{n}$ such that $f(\mathbf{x}+\mathbf{a})=g(\mathbf{x})$, else outputs ' $f$ and $g$ are not translation equivalent'. The running time of the algorithm is $\operatorname{poly}(n, d, \beta)$, where $\beta$ is the bit length of the coefficients of $f$ and $g$. 


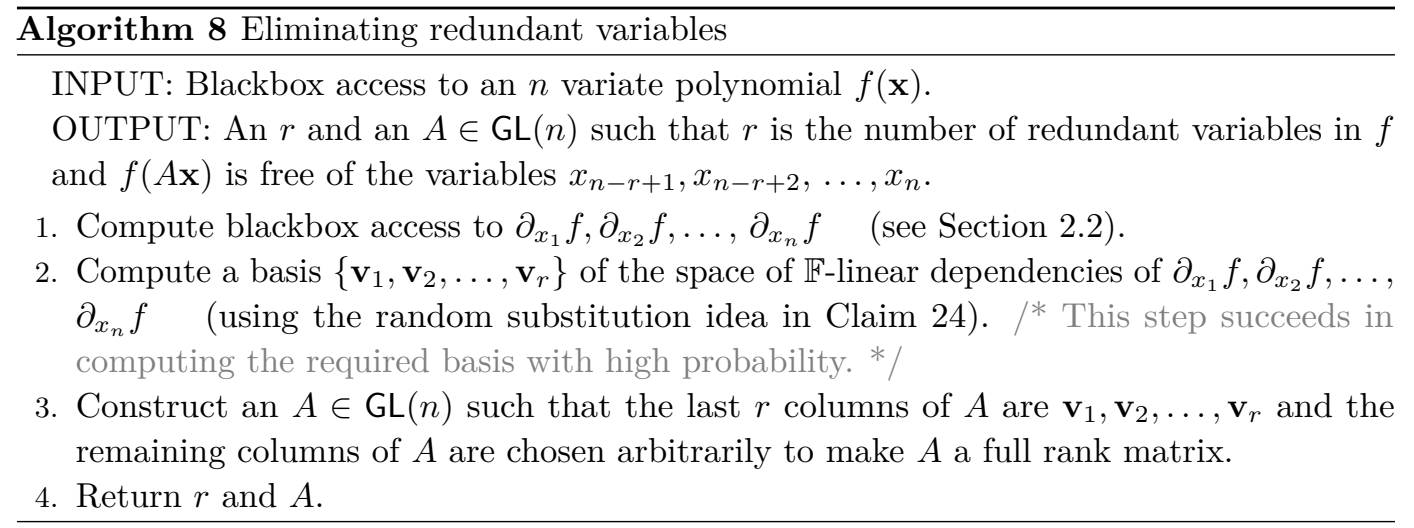

Proof. We present the algorithm formally in Algorithm 9. If it succeeds in computing a point $\mathbf{a} \in \mathbb{F}^{n}$ in the end (in step 20), it performs a randomized blackbox polynomial identity test (PIT) to check whether $f(\mathbf{x}+\mathbf{a})=g(\mathbf{x})$ (in step 22). If $f$ and $g$ are not translation equivalent, this final PIT finds it with probability at least $1-\frac{1}{\text { poly }(n)}$. So, for the analysis of the algorithm we can assume there is an $\mathbf{a}=\left(a_{1} a_{2} \ldots a_{n}\right)^{T} \in \mathbb{F}^{n}$ such that $f(\mathbf{x}+\mathbf{a})=g(\mathbf{x})$. The strategy outlined below helps to argue the correctness of Algorithm 9.

Strategy: Suppose $f(\mathbf{x}+\mathbf{a})=g(\mathbf{x})$. By equating the degree $d$ and degree $d-1$ homogeneous components of $f$ and $g$ we get the following equations,

$$
\begin{aligned}
& f^{[d]}=g^{[d]} \text { and } \\
& f^{[d-1]}+\sum_{i=1}^{n} a_{i} \cdot \frac{\partial f^{[d]}}{\partial x_{i}}=g^{[d-1]} \Rightarrow \sum_{i=1}^{n} a_{i} \cdot \frac{\partial f^{[d]}}{\partial x_{i}}=g^{[d-1]}-f^{[d-1]} .
\end{aligned}
$$

Let $f_{i}=\frac{\partial f^{[d]}}{\partial x_{i}}$ for $i \in[n]$. Blackbox access to the homogeneous components of $f: f^{[0]}, f^{[1]}, \ldots$, $f^{[d]}$, the homogeneous components of $g: g^{[0]}, g^{[1]}, \ldots, g^{[d]}$ and $f_{1}, f_{2}, \ldots f_{n}$ can be constructed from blackbox access to $f$ and $g$ in $\operatorname{poly}(n, d, \beta)$ time (see Section 2.2). If $f_{1}, f_{2}, \ldots, f_{n}$ are $\mathbb{F}$-linearly independent then with high probability over the random choices of $\mathbf{b}_{1}, \mathbf{b}_{2}, \ldots, \mathbf{b}_{n}$ $\in \mathbb{F}^{n}$ the matrix $\left(f_{j}\left(\mathbf{b}_{i}\right)\right)_{i, j \in[n]}$ has full rank (from Claim 24). Hence, we can solve for $a_{1}, a_{2}, \ldots, a_{n}$ uniquely from Equation (8). In the general case (when $f_{1}, f_{2}, \ldots, f_{n}$ may be $\mathbb{F}$-linearly dependent), the algorithm repeatedly applies variable reduction and degree reduction (as described below) to compute a.

Variable reduction. We construct a transformation $A \in \mathrm{GL}(n)$ such that $f^{[d]}(A \mathbf{x})$ has only the essential variables $x_{1}, \ldots, x_{m}$ (see Claim 26). Let $\tilde{f}=f(A \mathbf{x}), \tilde{g}=g(A \mathbf{x})$. It is sufficient to compute a point $\mathbf{b}=\left(\begin{array}{llll}b_{1} & b_{2} & \ldots & b_{n}\end{array}\right)^{T} \in \mathbb{F}^{n}$ such that $\tilde{f}(\mathbf{x}+\mathbf{b})=\tilde{g}(\mathbf{x})$ as

$$
\tilde{f}(\mathbf{x}+\mathbf{b})=\tilde{g}(\mathbf{x}) \Rightarrow f(A \mathbf{x}+A \mathbf{b})=g(A \mathbf{x}) \Rightarrow f(\mathbf{x}+A \mathbf{b})=g(\mathbf{x}) .
$$

So we can choose $\mathbf{a}=A \mathbf{b}$. As in Equation (8),

$$
\tilde{f}^{[d]}=\tilde{g}^{[d]} \quad \text { and } \quad \sum_{i=1}^{m} b_{i} \cdot \frac{\partial \tilde{f}^{[d]}}{\partial x_{i}}=\tilde{g}^{[d-1]}-\tilde{f}^{[d-1]} .
$$

The derivatives $\partial_{x_{i}} \tilde{f}^{[d]}$ for $i>m$ are zero as $\tilde{f}^{[d]}=f^{[d]}(A \mathbf{x})$ has only the essential variables $x_{1}, x_{2}, \ldots, x_{m}$. Also the polynomials $\left\{\partial_{x_{i}} \tilde{f}^{[d]}: i \in[m]\right\}$ are $\mathbb{F}$-linearly independent (by Claim 26). Hence, we can solve for unique $b_{1}, b_{2}, \ldots, b_{m}$ satisfying Equation (9) as before. 
Degree reduction. To compute $b_{m+1}, b_{m+2}, \ldots, b_{n}$ we reduce the problem to finding a point that asserts translation equivalence of two degree $d-1$ polynomials. Let $\mathbf{b}^{\prime}=$ $\left(\begin{array}{lllllll}b_{1} & b_{2} & \ldots & b_{m} & 0 & \ldots & 0\end{array}\right)^{T}, \widehat{f}=\tilde{f}\left(\mathbf{x}+\mathbf{b}^{\prime}\right)$. Further, let $\mathbf{e} \in \mathbb{F}^{n}$ such that $\hat{f}(\mathbf{x}+\mathbf{e})=\tilde{g}(\mathbf{x})$. Then the first $m$ coordinates of $\mathbf{e}$ must be zero ${ }^{38}$ and we can choose $\mathbf{b}=\mathbf{b}^{\prime}+\mathbf{e}$. We have the following equations,

$$
\begin{aligned}
& \widehat{f}^{[d]}(\mathbf{x}+\mathbf{e})+\left(\widehat{f}-\widehat{f}^{[d]}\right)(\mathbf{x}+\mathbf{e})=\tilde{g}^{[d]}(\mathbf{x})+\left(\tilde{g}-\tilde{g}^{[d]}\right)(\mathbf{x}) \\
& \Leftrightarrow \quad \tilde{f}^{[d]}(\mathbf{x}+\mathbf{e})+\left(\widehat{f}-\widehat{f}^{[d]}\right)(\mathbf{x}+\mathbf{e})=\tilde{g}^{[d]}(\mathbf{x})+\left(\tilde{g}-\tilde{g}^{[d]}\right)(\mathbf{x}) \quad\left(\text { as } \widehat{f}^{[d]}=\tilde{f}^{[d]}\right) .
\end{aligned}
$$

Since $\tilde{f}^{[d]}$ has only $x_{1}, x_{2}, \ldots, x_{m}$ variables and the first $m$ coordinates of $\mathbf{e}$ are zero, the above statement is equivalent to

$$
\begin{aligned}
& \tilde{f}^{[d]}(\mathbf{x})+\left(\widehat{f}-\widehat{f}^{[d]}\right)(\mathbf{x}+\mathbf{e})=\tilde{g}^{[d]}(\mathbf{x})+\left(\tilde{g}-\tilde{g}^{[d]}\right)(\mathbf{x}) \\
& \Leftrightarrow \quad\left(\widehat{f}-\widehat{f}^{[d]}\right)(\mathbf{x}+\mathbf{e})=\left(\tilde{g}-\tilde{g}^{[d]}\right)(\mathbf{x}) \quad(\text { from Equation (9)). }
\end{aligned}
$$

The polynomials $\widehat{f}-\widehat{f}^{[d]}$ and $\tilde{g}-\tilde{g}^{[d]}$ have degree at most $d-1$ and blackboxes for these polynomials can be constructed in poly $(n, d, \beta)$ time. Therefore the problem reduces to computing a point $\mathbf{e} \in \mathbb{F}^{n}$ that asserts translation equivalence of two degree $(d-1)$ polynomials.

Correctness of Algorithm 9: In steps 4-11, the algorithm carries out variable reduction and computes a part of the translation $\mathbf{b}$ that we call $\mathbf{b}^{\prime}$ in the above argument. The remaining part of $\mathbf{b}$ (which is the vector $\mathbf{e}$ above) is computed by carrying out degree reduction in step 12 and then inducting on lower degree polynomials. These parts are then added appropriately in step 17 , and finally an a is recovered in step 20 .

- Lemma 28 (restated). There is a randomized algorithm which when given blackbox access to an $n$ variate degree $d$ polynomial $f$, computes a basis of $\mathfrak{g}_{f}$ with probability at least $1-\frac{1}{\operatorname{poly}(n)}$ in time $\operatorname{poly}(n, d, \beta)$ where $\beta$ is the bit length of the coefficients in $f$.

Proof. Recall, the Lie algebra of $f$ is the set of all matrices $E=\left(e_{i j}\right)_{i, j \in[n]}$ such that $\sum_{i, j \in[n]} e_{i j} x_{j} \cdot \frac{\partial f}{\partial x_{i}}=0$. Hence, $\mathfrak{g}_{f}$ is the space of linear dependencies of the polynomials $x_{j} \cdot \frac{\partial f}{\partial x_{i}}$ for $i, j \in[n]$. Using Claim 23, we can derive blackboxes for these $n^{2}$ polynomials and then compute a basis of the space of linear dependencies with high probability using Claim 24.

\subsection{Proof of lemmas and claims in Section 3}

- Lemma 31 (restated). Let $\mathcal{W}_{1}, \mathcal{W}_{2}, \mathcal{W}_{3}$ be the following sets (spaces) of matrices:

1. $\mathcal{W}_{1}$ consists of all matrices $D=\left(d_{i j}\right)_{i, j \in[n]}$ such that $D$ is diagonal and

$$
\sum_{i=1}^{n} d_{i i} x_{i} \cdot \frac{\partial \mathrm{IMM}}{\partial x_{i}}=0
$$

2. $\mathcal{W}_{2}$ consists of all matrices $B=\left(b_{i j}\right)_{i, j \in[n]}$ such that

$$
\sum_{i, j \in[n]} b_{i j} x_{j} \cdot \frac{\partial \mathrm{IMM}}{\partial x_{i}}=0
$$

where in every summand $b_{i j} \neq 0$ only if $x_{i} \neq x_{j}$ and $x_{i}, x_{j} \in \mathbf{x}_{l}$ for some $l \in[d]$.

${ }^{38}$ As $b_{1}, b_{2}, \ldots, b_{m}$ can be solved uniquely. 


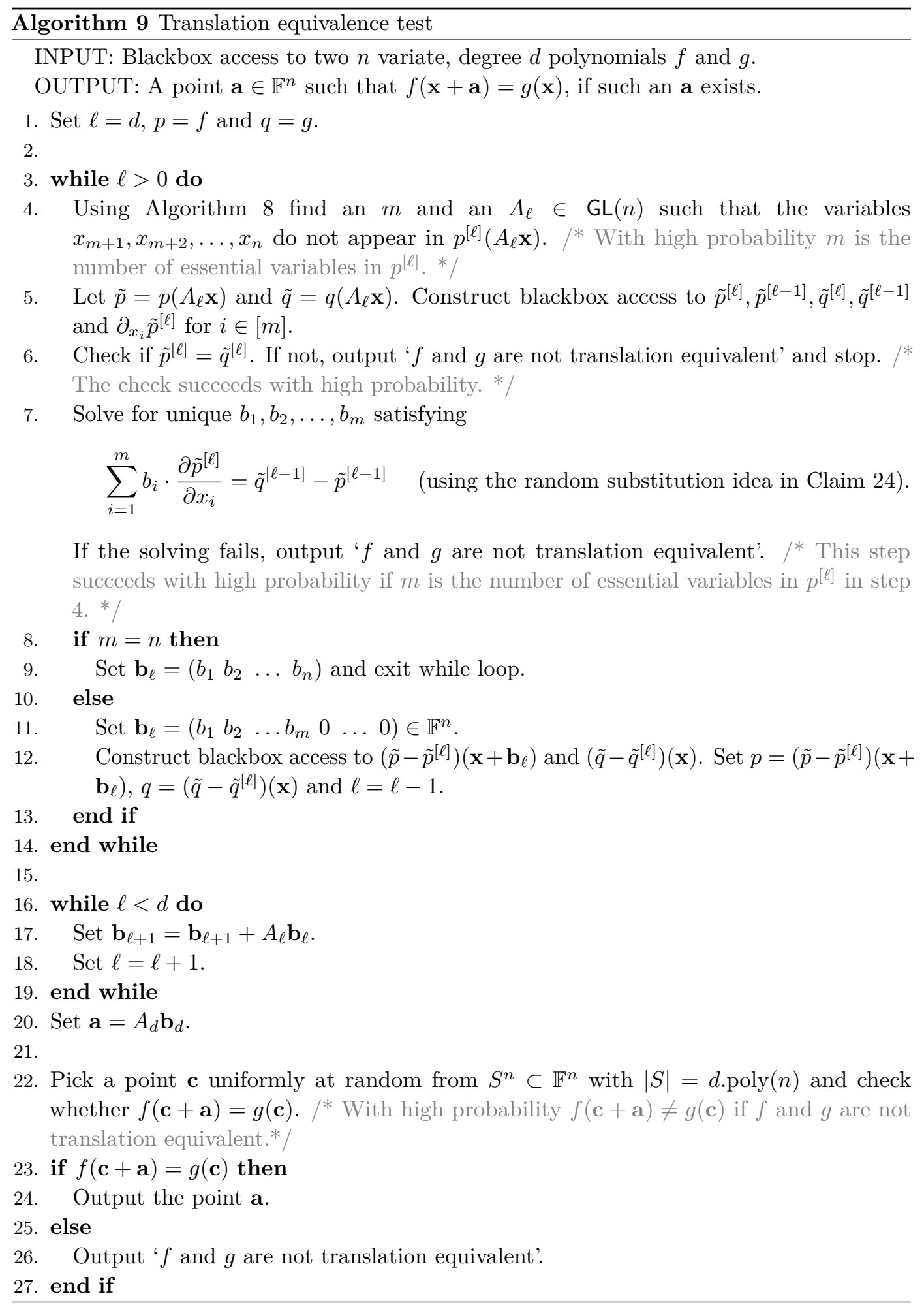


3. $\mathcal{W}_{3}$ consists of all matrices $C=\left(c_{i j}\right)_{i, j \in[n]}$ such that

$$
\sum_{i, j \in[n]} c_{i j} x_{j} \cdot \frac{\partial \mathrm{IMM}}{\partial x_{i}}=0
$$

where in every summand $c_{i j} \neq 0$ only if either $x_{i} \in \mathbf{x}_{2}, x_{j} \in \mathbf{x}_{1}$ or $x_{i} \in \mathbf{x}_{d-1}, x_{j} \in \mathbf{x}_{d}$. Then $\mathfrak{g}_{\mathrm{IMM}}=\mathcal{W}_{1} \oplus \mathcal{W}_{2} \oplus \mathcal{W}_{3}$.

Proof. Since $\mathcal{W}_{1} \cap \mathcal{W}_{2}=\left(\mathcal{W}_{1}+\mathcal{W}_{2}\right) \cap \mathcal{W}_{3}=\left\{\mathbf{0}_{n}\right\}$, where $\mathbf{0}_{n}$ is the $n \times n$ all zero matrix, it is sufficient to show $\mathfrak{g}_{\mathrm{MM}}=\mathcal{W}_{1}+\mathcal{W}_{2}+\mathcal{W}_{3}$. By definition, $\mathcal{W}_{1}+\mathcal{W}_{2}+\mathcal{W}_{3} \subseteq \mathfrak{g}_{\mathrm{MM}}$. We now show that $\mathfrak{g}_{\mathrm{MM}} \subseteq \mathcal{W}_{1}+\mathcal{W}_{2}+\mathcal{W}_{3}$. Let $E=\left(e_{i j}\right)_{i, j \in[n]}$ be a matrix in $\mathfrak{g}_{\mathrm{IMM}}$. Then $\sum_{i, j \in[n]} e_{i j} x_{j} \cdot \frac{\partial \mathrm{IMM}}{\partial x_{i}}=0$. We focus on a term $x_{j} \cdot \frac{\partial \mathrm{IMM}}{\partial x_{i}}$ and observe the following:

(a) If $x_{i}=x_{j}$ then the monomials of $x_{i} \cdot \frac{\partial \mathrm{IMM}}{\partial x_{i}}$ are also monomials of IMM. Such monomials do not appear in any term $x_{j} \cdot \frac{\partial \mathrm{IMM}}{\partial x_{i}}$, where $x_{i} \neq x_{j}$.

(b) If $x_{i} \neq x_{j}$ and $x_{i}, x_{j}$ belong to the same $\mathbf{x}_{l}$ then every monomial in $x_{j} \cdot \frac{\partial I M M}{\partial x_{i}}$ has exactly one variable from every $\mathbf{x}_{k}$ for $k \in[d]$. Such monomials do not appear in a term $x_{j} \cdot \frac{\partial \mathrm{IMM}}{\partial x_{i}}$, where $x_{i} \in \mathbf{x}_{l}$ and $x_{j} \in \mathbf{x}_{k}$ and $l \neq k$.

Due to this monomial disjointness, an equation $\sum_{i, j \in[n]} e_{i j} x_{j} \cdot \frac{\partial \mathrm{IMM}}{\partial x_{i}}=0$ corresponding to $E$ can be split into three equations:

1. $\sum_{i=1}^{n} d_{i i} x_{i} \cdot \frac{\partial \mathrm{IMM}}{\partial x_{i}}=0$.

2. $\sum_{i, j \in[n]} b_{i j} x_{j} \cdot \frac{\partial \mathrm{IMM}}{\partial x_{i}}=0$, where $b_{i j} \neq 0$ in a term only if $x_{i} \neq x_{j}$ and $x_{i}, x_{j} \in \mathbf{x}_{l}$ for some $l \in[d]$.

3. $\sum_{i, j \in[n]} c_{i j} x_{j} \cdot \frac{\partial \mathrm{IMM}}{\partial x_{i}}=0$, where $c_{i j} \neq 0$ in a term only if $x_{i} \in \mathbf{x}_{l}$ and $x_{j} \in \mathbf{x}_{k}$ for $l \neq k$.

Hence every $E=\left(e_{i j}\right)_{i, j \in[n]}$ in $\mathfrak{g}_{\mathrm{MM}}$ equals $D+B+C$ where

- $D \in \mathcal{W}_{1}$ is a diagonal matrix,

- $B \in \mathcal{W}_{2}$ is a block-diagonal ${ }^{39}$ matrix with diagonal entries zero,

- $C$ is a matrix with nonzero entries appearing outside the above block-diagonal.

To complete the proof of the lemma we show the following.

- Claim 61. Except those entries of $C$ whose rows and columns are indexed by $\mathbf{x}_{2}$ and $\mathbf{x}_{1}$ variables respectively, or $\mathbf{x}_{d-1}$ and $\mathbf{x}_{d}$ variables respectively, all the other entries are zero.

Proof. In a term $x_{p q}^{(l)} \cdot \frac{\partial \mathrm{IMM}}{\partial x_{i j}^{(k)}}$ where $l \neq k$, every monomial has two variables from $\mathbf{x}_{l}$ and no variable from $\mathbf{x}_{k}$. Hence from the equation corresponding to $C$ we get separate equations for every pair $(l, k)$ due to monomial disjointness:

$$
\sum_{p \in\left[w_{l-1}\right], q \in\left[w_{l}\right]} \sum_{i \in\left[w_{k-1}\right], j \in\left[w_{k}\right]} c_{p q, i j} x_{p q}^{(l)} \cdot \frac{\partial \mathrm{IMM}}{\partial x_{i j}^{(k)}}=0, \quad \text { where } l \neq k .
$$

Collecting coefficients corresponding to $\frac{\partial \mathrm{IMM}}{\partial x_{i j}^{(k)}}$ in the above equation we get

$$
\sum_{i \in\left[w_{k-1}\right], j \in\left[w_{k}\right]} \ell_{i j}^{(k)} \cdot \frac{\partial \mathrm{IMM}}{\partial x_{i j}^{(k)}}=0, \quad \text { where } \ell_{i j}^{(k)} \text { is a linear form in the variables from } \mathbf{x}_{l} .
$$

Figure 6 depicts a term $\ell_{i j}^{(k)} \cdot \frac{\partial \mathrm{IMM}}{\partial x_{i j}^{(k)}}$ using an ABP that computes it. So the LHS of the above equation can be computed by an ABP B that has edge labels identical to that of the ABP for IMM, except for the edges in layer $k$. The $(i, j)$-th edge of layer $k$ in B is labelled by $\ell_{i j}^{(k)}$.

\footnotetext{
${ }^{39}$ An entry is in the block-diagonal if and only if the variables labelling the row and column of the entry are in the same $\mathbf{x}_{l}$ for some $l \in[d]$.
} 


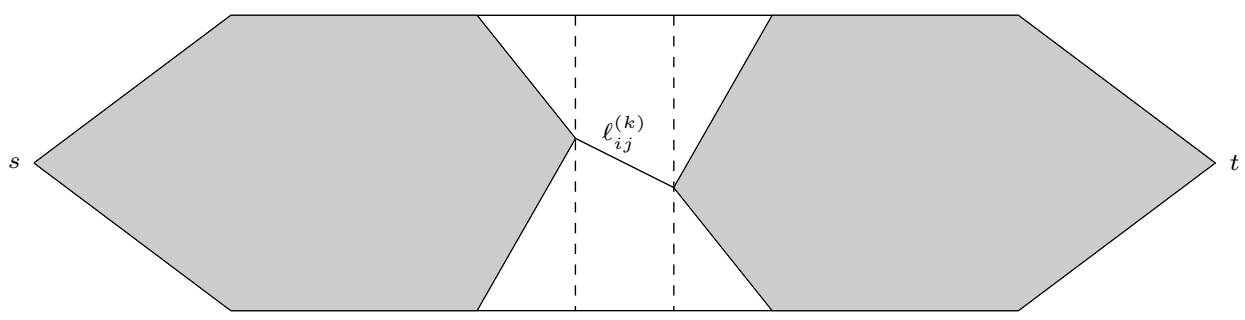

Figure 6 An ABP computing the term $\ell_{i j}^{(k)} \cdot \frac{\partial \mathrm{IMM}}{\partial x_{i j}^{(k)}}$.

Suppose $\ell_{i j}^{(k)} \neq 0$ and the coefficient of the variable $x_{p q}^{(l)}$ in $\ell_{i j}^{(k)}$ is nonzero, i.e. $c_{p q, i j} \neq 0$. If $(l, k)$ is neither $(1,2)$ nor $(d, d-1)$ then the assumption $c_{p q, i j} \neq 0$ leads to a contradiction as follows.

Consider an $s$ to $t$ path $P$ in B that goes through the $(i, j)$-th edge of layer $k$ (which is labelled by $\left.\ell_{i j}^{(k)}\right)$ but excludes the $(p, q)$-th edge of layer $l$ (which is labelled by $\left.x_{p q}^{(l)}\right)$, the $(p, i)$-th edge of layer $k-1$ if $l=k-1$ and the $(j, q)$-th edge of layer $k+1$ if $l=k+1$ (we can notice this is always possible since $(l, k)$ is neither $(1,2)$ nor $(d, d-1))$. Then, if we retain the variables labelling the edges of $P$ ouside the layer $k$ and the variable $x_{p q}^{(l)}$, and set every other variable to zero then $P$ becomes the unique $s$ to $t$ path in $\mathrm{B}$ with nonzero weight (since $c_{p q, i j} \neq 0$ ). But this contradicts the fact that ABP B is computing an identically zero polynomial (by Equation (10)).

Therefore, $\mathfrak{g}_{\mathrm{MM}} \subseteq \mathcal{W}_{1}+\mathcal{W}_{2}+\mathcal{W}_{3}$ implying $\mathfrak{g}_{\mathrm{MM}}=\mathcal{W}_{1} \oplus \mathcal{W}_{2} \oplus \mathcal{W}_{3}$

- Lemma 32 (restated). The space $\mathcal{W}_{3}=\mathcal{W}_{3}^{(a)} \oplus \mathcal{W}_{3}^{(b)}$ where $\mathcal{W}_{3}^{(a)}=\mathcal{A}_{1} \oplus \mathcal{A}_{2} \oplus \cdots \oplus \mathcal{A}_{w_{2}}$ and $\mathcal{W}_{3}^{(b)}=\mathcal{A}_{1}^{\prime} \oplus \mathcal{A}^{\prime}{ }_{2} \oplus \cdots \oplus \mathcal{A}^{\prime}{ }_{w_{d-2}}$ such that for every $i \in\left[w_{2}\right] \mathcal{A}_{i}$ is isomorphic to the space of $w_{1} \times w_{1}$ anti-symmetric matrices over $\mathbb{F}$, and for every $j \in\left[w_{d-2}\right] \mathcal{A}^{\prime}{ }_{j}$ is isomorphic to the space of $w_{d-1} \times w_{d-1}$ anti-symmetric matrices over $\mathbb{F}$. Hence $\operatorname{dim}\left(\mathcal{W}_{3}\right)=$ $\frac{1}{2}\left[w_{1} w_{2}\left(w_{1}-1\right)+w_{d-1} w_{d-2}\left(w_{d-1}-1\right)\right]$.

Proof. Recall, $\mathcal{W}_{3}$ is the space of all matrices $C=\left(c_{i j}\right)_{i, j \in[n]}$ such that

$$
\sum_{i, j \in[n]} c_{i j} x_{j} \cdot \frac{\partial \mathrm{IMM}}{\partial x_{i}}=0
$$

where in every nonzero summand either $x_{i} \in \mathbf{x}_{2}, x_{j} \in \mathbf{x}_{1}$ or $x_{i} \in \mathbf{x}_{d-1}, x_{j} \in \mathbf{x}_{d}$. In Equation (11) every monomial in a term $x_{p}^{(1)} \cdot \frac{\partial \mathrm{IMM}}{\partial x_{q r}^{(2)}}$ has two variables from $\mathbf{x}_{1}$. Similarly, every monomial in a term $x_{p}^{(d)} \cdot \frac{\partial \mathrm{IMM}}{\partial x_{q r}^{(d-1)}}$ has two variables from $\mathbf{x}_{d}$ respectively. Owing to monomial disjointness, Equation (11) gives two equations

$$
\begin{gathered}
\sum_{r \in\left[w_{2}\right]} \sum_{p, q \in\left[w_{1}\right]} c_{p q r}^{(1)} x_{p}^{(1)} \cdot \frac{\partial \mathrm{IMM}}{\partial x_{q r}^{(2)}}=0, \quad \text { and } \\
\sum_{q \in\left[w_{d-2}\right]} \sum_{p, r \in\left[w_{d-1}\right]} c_{p q r}^{(d)} x_{p}^{(d)} \cdot \frac{\partial \mathrm{IMM}}{\partial x_{q r}^{(d-1)}}=0 .
\end{gathered}
$$

Thus $\mathcal{W}_{3}=\mathcal{W}_{3}^{(a)} \oplus \mathcal{W}_{3}^{(b)}$ where $\mathcal{W}_{3}^{(a)}$ consists of matrices satisfying Equation (12) and $\mathcal{W}_{3}^{(b)}$ consists of matrices satisfying Equation (13). We argue the following about $\mathcal{W}_{3}^{(a)}$. 


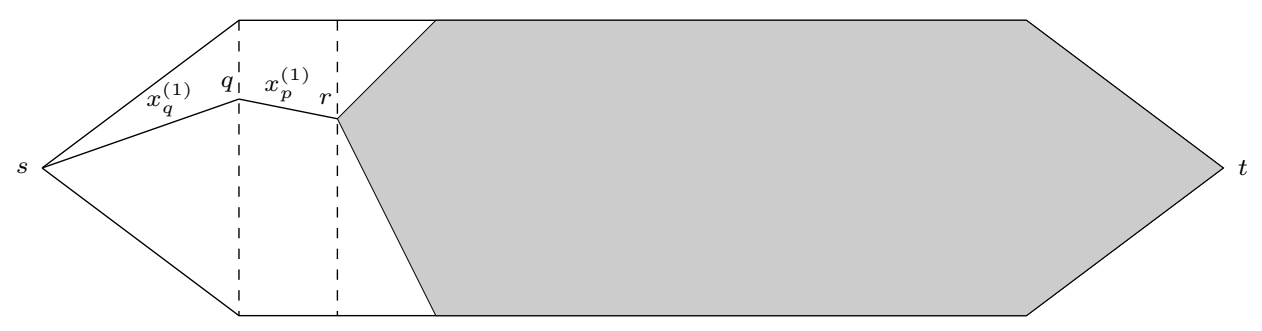

Figure 7 An ABP computing the term $x_{p}^{(1)} \cdot \frac{\partial \mathrm{IMM}}{\partial x_{q r}^{(2)}}$.

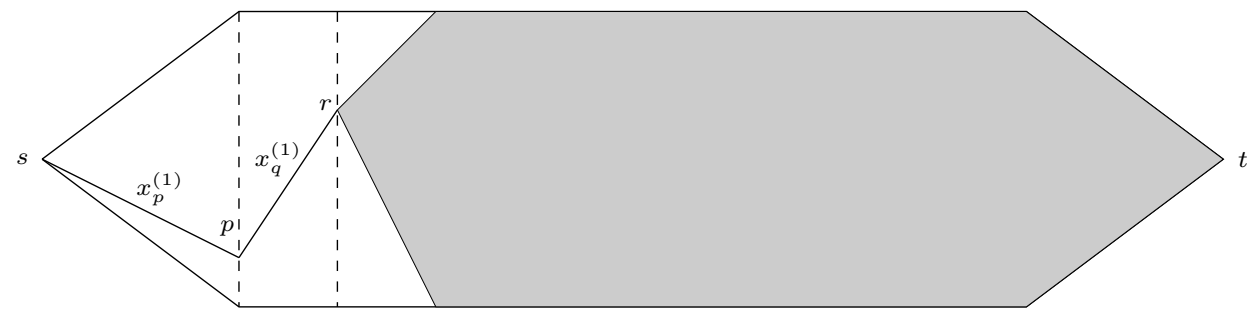

Figure 8 An ABP computing the term $x_{q}^{(1)} \cdot \frac{\partial I M M}{\partial x_{p r}^{(2)}}$.

- Claim 62. $\mathcal{W}_{3}^{(a)}=\mathcal{A}_{1} \oplus \mathcal{A}_{2} \oplus \cdots \oplus \mathcal{A}_{w_{2}}$ where every $\mathcal{A}_{i}$ is isomorphic to the space of $w_{1} \times w_{1}$ anti-symmetric matrices over $\mathbb{F}$.

Proof. Figure 7 depicts an ABP computing the term $x_{p}^{(1)} \cdot \frac{\partial \mathrm{IMM}}{\partial x_{q r}^{(2)}}$. Every monomial in $c_{p q r}^{(1)} x_{p}^{(1)} \cdot \frac{\partial \mathrm{IMM}}{\partial x_{q r}^{(2)}}$ is divisible by $x_{p}^{(1)} x_{q}^{(1)}$.

The only other term in Equation (12) that contains monomials divisible by $x_{p}^{(1)} x_{q}^{(1)}$ is $c_{q p r}^{(1)} x_{q}^{(1)} \cdot \frac{\partial \mathrm{IMM}}{\partial x_{p r}^{(2)}}$. Figure 8 depicts an ABP computing $x_{q}^{(1)} \cdot \frac{\partial \mathrm{IMM}}{\partial x_{p r}^{(2)}}$.

Since the terms in Figures 7 and 8 have no monomials in common with any other term in Equation (12) it must be that $c_{p q r}^{(1)}=-c_{q p r}^{(1)}$. Moreover, if $p=q$ then $c_{p q r}^{(1)}=0$. Thus Equation (12) gives an equation for every $r \in\left[w_{2}\right]$

$$
\sum_{p, q \in\left[w_{1}\right], p \neq q} c_{p q r}^{(1)} x_{p}^{(1)} \cdot \frac{\partial \mathrm{IMM}}{\partial x_{q r}^{(2)}}=0
$$

such that the matrix $C_{r}=\left(c_{p q r}^{(1)}\right)_{p, q \in\left[w_{1}\right]} \in \mathbb{F}^{w_{1} \times w_{1}}$ is anti-symmetric. Further any antisymmetric matrix can be used to get an equation like Equation (14). Thus, as shown in Figure 9, every matrix $C^{(a)} \in \mathcal{W}_{3}^{(a)}$ is such that for every $r \in\left[w_{2}\right]$, the $w_{1} \times w_{1}$ submatrix (say $C_{r}^{(a)}$ ) defined by the rows labelled by the $x_{q r}^{(2)}$ variables and the columns labelled by the $x_{p}^{(1)}$ variables for $p, q \in\left[w_{1}\right]$ is anti-symmetric.

Also, any matrix satisfying the above properties belongs to $\mathcal{W}_{3}^{(a)}$. Naturally, if we define $\mathcal{A}_{r}$ to be the space of $n \times n$ matrices such that the $w_{1} \times w_{1}$ submatrix defined by the rows labelled by the $x_{q r}^{(2)}$ variables and the columns labelled by the $x_{p}^{(1)}$ variables for $p, q \in\left[w_{1}\right]$ is anti-symmetric and all other entries are zero then $\mathcal{W}_{3}^{(a)}=\mathcal{A}_{1} \oplus \mathcal{A}_{2} \oplus \cdots \oplus \mathcal{A}_{w_{2}}$.

Similarly, it can be shown that $\mathcal{W}_{3}^{(b)}=\mathcal{A}^{\prime}{ }_{1} \oplus \mathcal{A}^{\prime}{ }_{2} \oplus \cdots \oplus \mathcal{A}^{\prime}{ }_{w_{d-2}}$ where every $\mathcal{A}^{\prime}{ }_{i}$ is isomorphic to the space of $w_{d-1} \times w_{d-1}$ anti-symmetric matrices. This completes the proof of Lemma 32 . 


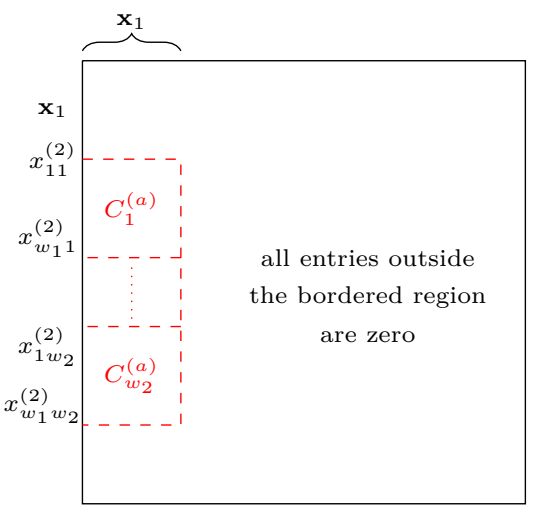

Figure 9 A matrix $C^{(a)}$ in $\mathcal{W}_{3}^{(a)}$.

Lemma 33 (restated). The space $\mathcal{W}_{2}=\mathcal{B}_{1} \oplus \mathcal{B}_{2} \oplus \cdots \oplus \mathcal{B}_{d-1}$ such that for every $k \in[d-1]$, $\mathcal{B}_{k}$ is isomorphic to the $\mathbb{F}$-linear space spanned by $t_{k} \times t_{k}$ matrices of the form

$$
\left[\begin{array}{cc}
-Z^{T} \otimes I_{w_{k-1}} & 0 \\
0 & I_{w_{k+1}} \otimes Z
\end{array}\right]_{t_{k} \times t_{k}} \quad \text { where } Z \in \mathcal{Z}_{w_{k}} \text { and } t_{k}=w_{k}\left(w_{k-1}+w_{k+1}\right) .
$$

Hence, $\operatorname{dim}\left(\mathcal{W}_{2}\right)=\sum_{k=1}^{d-1}\left(w_{k}^{2}-w_{k}\right)$.

Proof. Recall $w_{0}=w_{d}=1$ and $\mathcal{Z}_{w_{k}}$ denotes the space of $w_{k} \times w_{k}$ matrix with diagonal entries 0 , and $\mathcal{W}_{2}$ is the space of all matrices $B=\left(b_{i j}\right)_{i, j \in[n]}$ such that

$$
\sum_{i, j \in[n]} b_{i j} x_{j} \cdot \frac{\partial \mathrm{IMM}}{\partial x_{i}}=0
$$

where in every term $b_{i j} \neq 0$ only if $x_{i} \neq x_{j}$ and $x_{i}, x_{j} \in \mathbf{x}_{l}$ for some $l \in[d]$. The following observation is easy to verify.

Observation 63. Suppose $l \in[2, d-1]$. A term $x_{i_{1} j_{1}}^{(l)} \cdot \frac{\partial \mathrm{IMM}}{\partial x_{i_{2} j_{2}}^{(l)}}$ where $i_{1} \neq i_{2}$ and $j_{1} \neq j_{2}$ does not share a monomial with any other term in Equation (15).

Hence for $l \in[2, d-1]$, terms of the kind $x_{i_{1} j_{1}}^{(l)} \cdot \frac{\partial \mathrm{IMM}}{\partial x_{i_{2} j_{2}}^{(l)}}$ where $i_{1} \neq i_{2}$ and $j_{1} \neq j_{2}$ are absent in Equation (15). A monomial appearing in a nonzero term of Equation (15) is of the form $x_{i_{1}}^{(1)} \cdot x_{i_{1} i_{2}}^{(2)} \cdots x_{i_{k-1} i_{k}}^{(k)} \cdot x_{i_{k}^{\prime} i_{k+1}}^{(k+1)} \cdots x_{i_{d-1} i_{d}}^{(d-1)} \cdot x_{i_{d}}^{(d)}$ where $i_{k} \neq i_{k}^{\prime}$, for some $k \in[d-1]$. We say such a monomial is broken at the $k$-th interface. Observe the following.

- Observation 64. The terms $x_{p r}^{(k)} \cdot \frac{\partial \mathrm{IMM}}{\partial x_{p q}^{(k)}}$ where $p \in\left[w_{k-1}\right], q, r \in\left[w_{k}\right], q \neq r$, and $x_{m j}^{(k+1)} \cdot \frac{\partial \mathrm{IMM}}{\partial x_{i j}^{(k+1)}}$ where $i, m \in\left[w_{k}\right], j \in\left[w_{k+1}\right], i \neq m$ are the only two whose monomials are broken at the $k$-th interface.

Thus from Equation $(15)$ we get $(d-1)$ equations one for each interface by considering cancellations of monomials broken at that interface. For $k \in[2, d-2]$, let $\mathcal{B}_{k}$ be the space of all $n \times n$ matrices $B_{k}$ such that

1. the entry corresponding to the row labelled by $x_{p q}^{(k)}$ and the column labelled by $x_{p r}^{(k)}$ is $b_{p q, p r}^{(k)} \in \mathbb{F}$ for $p \in\left[w_{k-1}\right], q, r \in\left[w_{k}\right]$ and $q \neq r$,

2. the entry corresponding to the row labelled by $x_{i j}^{(k+1)}$ and the column labelled by $x_{m j}^{(k+1)}$ is $b_{i j, m j}^{(k+1)} \in \mathbb{F}$ for $i, m \in\left[w_{k}\right], j \in\left[w_{k+1}\right]$ and $i \neq m$, 
3. all other entries of $B_{k}$ are zero, and

4.

$$
\sum_{p \in\left[w_{k-1}\right],} b_{q, r \in\left[w_{k}\right],}^{(k)} x_{q \neq, p r} x_{p r}^{(k)} \cdot \frac{\partial \mathrm{IMM}}{\partial x_{p q}^{(k)}}+\sum_{i, m \in\left[w_{k}\right],} \sum_{j \in\left[w_{k+1}\right], i \neq m} b_{i j, m j}^{(k+1)} x_{m j}^{(k+1)} \cdot \frac{\partial \mathrm{IMM}}{\partial x_{i j}^{(k+1)}}=0 .
$$

We can define spaces $\mathcal{B}_{1}$ and $\mathcal{B}_{d-1}$ similarly considering monomials broken at the first and the last interface respectively. As Equation (15) can be split into $(d-1)$ equations, one for every interface, $\mathcal{W}_{2}=\mathcal{B}_{1}+\mathcal{B}_{2}+\cdots+\mathcal{B}_{d-1}$. Since the spaces $\mathcal{B}_{1}, \ldots, \mathcal{B}_{d-1}$ control different entries of $n \times n$ matrices, $\mathcal{W}_{2}=\mathcal{B}_{1} \oplus \mathcal{B}_{2} \oplus \cdots \oplus \mathcal{B}_{d-1}$.

- Claim 65. For $k \in[2, d-2], \mathcal{B}_{k}$ is isomorphic to the $\mathbb{F}$-linear space spanned by $t_{k} \times t_{k}$ matrices of the form

$$
\left[\begin{array}{cc}
-Z^{T} \otimes I_{w_{k-1}} & 0 \\
0 & I_{w_{k+1}} \otimes Z
\end{array}\right]_{t_{k} \times t_{k}} \quad \text { where } Z \in \mathcal{Z}_{w_{k}} \text { and } t_{k}=w_{k}\left(w_{k-1}+w_{k+1}\right) .
$$

Proof. Collecting same derivative terms in Equation (16) we get

$$
\sum_{p \in\left[w_{k-1}\right], q \in\left[w_{k}\right]} \ell_{p q}^{(k)} \cdot \frac{\partial \mathrm{IMM}}{\partial x_{p q}^{(k)}}+\sum_{i \in\left[w_{k}\right], j \in\left[w_{k+1}\right]} \ell_{i j}^{(k+1)} \cdot \frac{\partial \mathrm{IMM}}{\partial x_{i j}^{(k+1)}}=0,
$$

where $\ell_{p q}^{(k)}$ is a linear form containing variables $x_{p r}^{(k)}$ such that $r \neq q$, and $\ell_{i j}^{(k+1)}$ is a linear form containing variables $x_{m j}^{(k+1)}$ such that $m \neq i$. Here is a succinct way to write Equation (17):

$$
Q_{1} \cdot Q_{2} \cdots Q_{k}^{\prime} \cdot Q_{k+1} \cdot Q_{k+2} \cdots Q_{d-1} \cdot Q_{d}+Q_{1} \cdot Q_{2} \cdots Q_{k} \cdot Q_{k+1}^{\prime} \cdot Q_{k+2} \cdots Q_{d-1} \cdot Q_{d}=0,
$$

where $Q_{1}, \ldots, Q_{d}$ are matrices as in Section $2.3, Q_{k}^{\prime}=\left(\ell_{p q}^{(k)}\right)_{p \in\left[w_{k-1}\right], q \in\left[w_{k}\right]}$ and $Q_{k+1}^{\prime}=$ $\left(\ell_{i j}^{(k+1)}\right)_{i \in\left[w_{k}\right], j \in\left[w_{k+1}\right]}$. This implies

$$
Q_{k}^{\prime} \cdot Q_{k+1}+Q_{k} \cdot Q_{k+1}^{\prime}=0
$$

as $Q_{1}, \ldots, Q_{d}$ have distinct sets of variables, and the variables appearing in $Q_{k}^{\prime}$ and $Q_{k+1}^{\prime}$ are the same as in $Q_{k}$ and $Q_{k+1}$ respectively. The variable disjointness of $Q_{k}$ and $Q_{k+1}$ can be exploited to infer $Q_{k+1}^{\prime}=Z \cdot Q_{k+1}$ and $Q_{k}^{\prime}=-Q_{k} \cdot Z$ where $Z$ is in $\mathbb{F}^{w_{k} \times w_{k}}$ (even if $Q_{k}, Q_{k+1}$ may not be square matrices). As the linear form $\ell_{p q}^{(k)}$ is devoid of the variable $x_{p q}^{(k)}$, it must be that $Z \in \mathcal{Z}_{w_{k}}$. Moreover, any $Z \in \mathcal{Z}_{w_{k}}$ can be used along with the relations $Q_{k+1}^{\prime}=Z \cdot Q_{k+1}$ and $Q_{k}^{\prime}=-Q_{k} \cdot Z$ to satisfy Equation (18) and hence also Equations (16) and (17).

Let $Z=\left(z_{i m}\right)_{i, m \in\left[w_{k}\right]}$. Since $Q_{k+1}^{\prime}=Z \cdot Q_{k+1}$, the coefficient of $x_{m j}^{(k+1)}$ in $\ell_{i j}^{(k+1)}$ is $z_{i m}$ for every $j \in\left[w_{k+1}\right]$. Hence in Equation $(16), b_{i j, m j}^{(k+1)}=z_{i m}$ for every $j \in\left[w_{k+1}\right]$. Similarly, since $Q_{k}^{\prime}=-Q_{k} \cdot Z$ the coefficient of $x_{p r}^{(k)}$ in $\ell_{p q}^{(k)}$ is $-z_{r q}$ for every $p \in\left[w_{k-1}\right]$. Hence in Equation (16) $b_{p q, p r}^{(k)}=-z_{r q}$ for every $p \in\left[w_{k-1}\right]$. Thus the submatrix of $B_{k}$ defined by the rows and columns labelled by the variables in $\mathbf{x}_{k}$ and $\mathbf{x}_{k+1}$ looks like

$$
\left[\begin{array}{cc}
-Z^{T} \otimes I_{w_{k-1}} & 0 \\
0 & I_{w_{k+1}} \otimes Z
\end{array}\right]_{t_{k} \times t_{k}}
$$

where $t_{k}=w_{k}\left(w_{k-1}+w_{k+1}\right)$ and all other entries in $B_{k}$ are zero. Hence $\mathcal{B}_{k}$ is isomorphic to the space generated by $t_{k} \times t_{k}$ matrices of the above kind. This proves the claim. 
We can similarly show that $\mathcal{B}_{1}$ is isomorphic to the space generated by square matrices of the form

$$
\left[\begin{array}{cc}
-Z^{T} & 0 \\
0 & I_{w_{2}} \otimes Z
\end{array}\right]_{t_{1} \times t_{1}} \quad \text { where } Z \in \mathcal{Z}_{w_{1}} \text { and } t_{1}=w_{1}+w_{1} w_{2}
$$

and $\mathcal{B}_{d-1}$ is isomorphic to the space generated by square matrices of the form

$$
\left[\begin{array}{cc}
-Z^{T} \otimes I_{w_{d-2}} & 0 \\
0 & Z
\end{array}\right]_{t_{d-1} \times t_{d-1}} \quad \text { where } Z \in \mathcal{Z}_{w_{d-1}} \text { and } t_{d-1}=w_{d-1} w_{d-2}+w_{d-1} .
$$

This completes the proof of Lemma 33.

- Lemma 34 (restated). The space $\mathcal{W}_{1}$ contains the space $\mathcal{D}_{1} \oplus \mathcal{D}_{2} \oplus \cdots \oplus \mathcal{D}_{d-1}$ such that for every $k \in[d-1], \mathcal{D}_{k}$ is isomorphic to the $\mathbb{F}$-linear space spanned by $t_{k} \times t_{k}$ matrices of the form

$$
\left[\begin{array}{cc}
-Y \otimes I_{w_{k-1}} & 0 \\
0 & I_{w_{k+1}} \otimes Y
\end{array}\right]_{t_{k} \times t_{k}} \quad \text { where } Y \in \mathcal{Y}_{w_{k}} \text { and } t_{k}=w_{k}\left(w_{k-1}+w_{k+1}\right) .
$$

Hence, $\operatorname{dim}\left(\mathcal{W}_{1}\right) \geq \sum_{k=1}^{d-1} w_{k}$.

Proof. The proof is similar to the proof of Lemma 33. Recall $w_{0}=w_{d}=1$ and $\mathcal{Y}_{w_{k}}$ denotes the space of $w_{k} \times w_{k}$ diagonal matrices. Every $D \in \mathcal{W}_{1}$ satisfies an equation of the following form

$$
\sum_{i \in\left[w_{1}\right]} d_{i}^{(1)} x_{i}^{(1)} \cdot \frac{\partial \mathrm{IMM}}{\partial x_{i}^{(1)}}+\sum_{k=2}^{d-1} \sum_{i \in\left[w_{k-1}\right], j \in\left[w_{k}\right]} d_{i j}^{(k)} x_{i j}^{(k)} \cdot \frac{\partial \mathrm{IMM}}{\partial x_{i j}^{(k)}}+\sum_{i \in\left[w_{d-1}\right]} d_{i}^{(d)} x_{i}^{(d)} \cdot \frac{\partial \mathrm{IMM}}{\partial x_{i}^{(d)}}=0 .
$$

A succinct way to write the above equation is

$$
\sum_{k=1}^{d} Q_{1} Q_{2} \cdots Q_{k-1} Q_{k}^{\prime} Q_{k+1} \cdots Q_{d}=0
$$

where $Q_{1}^{\prime}=\left(d_{i}^{(1)} x_{i}^{(1)}\right)_{i \in\left[w_{1}\right]}$ is a row vector, $Q_{d}^{\prime}=\left(d_{i}^{(d)} x_{i}^{(d)}\right)_{i \in\left[w_{d-1}\right]}^{T}$ is a column vector, $Q_{k}^{\prime}=\left(d_{i j}^{(k)} x_{i j}^{(k)}\right)_{i \in\left[w_{k-1}\right], j \in\left[w_{k}\right]}$, and $Q_{1}, \ldots, Q_{d}$ are matrices as in Section 2.3. For every $k \in[d-1]$, let us focus on those $D_{k} \in \mathcal{W}_{1}$ for which the matrices $Q_{1}^{\prime}, \ldots, Q_{k-1}^{\prime}, Q_{k+2}^{\prime}, \ldots, Q_{d}^{\prime}$ are zero in Equation (19). Such a $D_{k}$ satisfies the following equation,

$$
Q_{1} \cdot Q_{2} \cdots Q_{k}^{\prime} \cdot Q_{k+1} \cdots Q_{d}+Q_{1} \cdot Q_{2} \cdots Q_{k} \cdot Q_{k+1}^{\prime} \cdots Q_{d}=0 .
$$

Using a similar argument as in the proof of Lemma 33 we get $Q_{k+1}^{\prime}=Y \cdot Q_{k+1}$ and $Q_{k}^{\prime}=-Q_{k} \cdot Y$ where $Y \in \mathcal{Y}_{w_{k}}$. Further, any $Y \in \mathcal{Y}_{w_{k}}$ can be used along with the relations $Q_{k+1}^{\prime}=Y \cdot Q_{k+1}$ and $Q_{k}^{\prime}=-Q_{k} \cdot Y$ to satisfy Equation (20). The set of $D_{k} \in \mathcal{W}_{1}$ satisfying Equation (20) forms an $\mathbb{F}$-linear space; call it $\mathcal{D}_{k}$. Every $D_{k} \in \mathcal{D}_{k}$ is such that the submatrix defined by the rows and the columns labelled by the variables in $\mathbf{x}_{k}$ and $\mathbf{x}_{k+1}$ looks like

$$
\left[\begin{array}{cc}
-Y \otimes I_{w_{k-1}} & 0 \\
0 & I_{w_{k+1}} \otimes Y
\end{array}\right]_{t_{k} \times t_{k}} \quad \text { where } Y \in \mathcal{Y}_{w_{k}} \text { and } t_{k}=w_{k}\left(w_{k-1}+w_{k+1}\right)
$$

and all other entries in $D_{k}$ are zero. Moreover, any $n \times n$ matrix with this structure is in $\mathcal{D}_{k}$. Thus $\mathcal{D}_{k}$ is isomorphic to the space of all $t_{k} \times t_{k}$ matrices of the form shown above. It can also be easily verified that every matrix in $\mathcal{D}_{1}+\ldots+\mathcal{D}_{d-1}$ can be expressed uniquely as a sum of matrices in these spaces. Hence $\mathcal{W}_{1} \supseteq \mathcal{D}_{1} \oplus \mathcal{D}_{2} \oplus \cdots \oplus \mathcal{D}_{d-1}$ completing the proof of Lemma 34. 


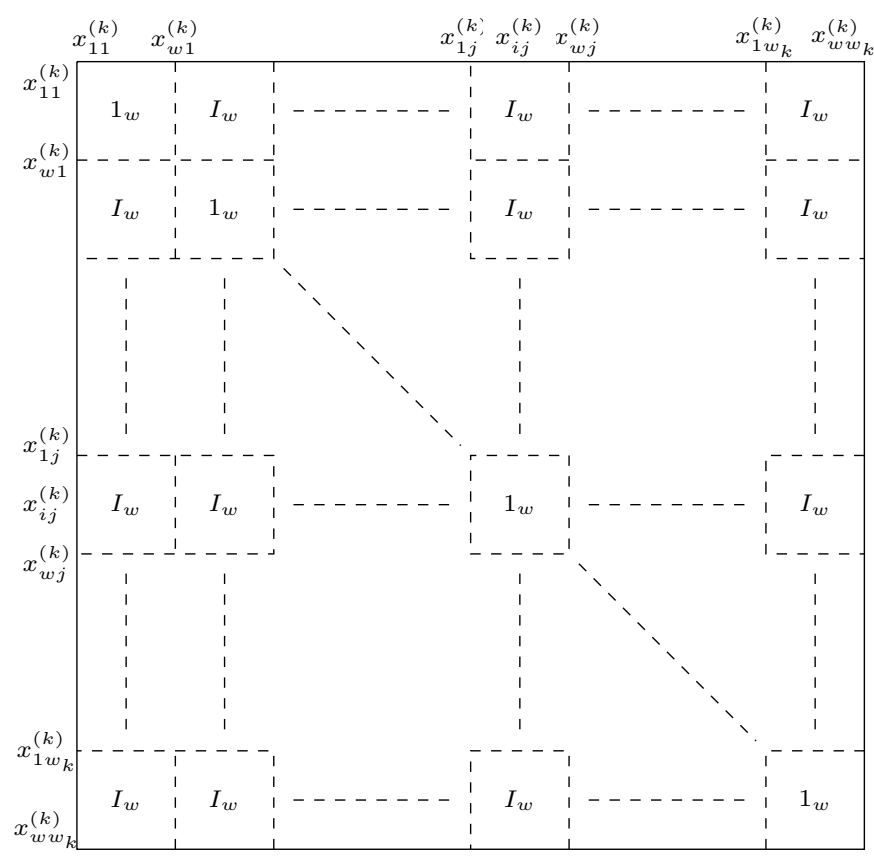

Figure 10 Submatrix of $L$ restricted to rows/columns indexed by $\mathbf{x}_{k}$.

- Claim 40 (restated). No invariant subspace of $\mathfrak{g}_{\mathrm{IMM}}$ is properly contained in $\mathcal{U}_{k}$ for $k \in$ $[2, d-1]$.

Proof. Let $\mathcal{U} \subseteq \mathcal{U}_{k}$ be an invariant subspace of $\mathfrak{g}_{\mathrm{ммм}}$. From Claim 38 it follows that $\mathcal{U}$ is a coordinate subspace. For $t \in \mathbb{N}$, let $\tilde{1}_{t} \stackrel{\text { def }}{=} 1_{t}-I_{t}$, where $1_{t}$ is the $t \times t$ all one matrix. From Lemma 33 , there are matrices $B_{k-1}$ and $B_{k}$ in $\mathfrak{g}_{\mathrm{MM}}$ such that the submatrix of $B_{k-1}$ restricted to the rows and the columns labelled by the variables in $\mathbf{x}_{k-1} \uplus \mathbf{x}_{k}$ looks like

$$
\left[\begin{array}{cc}
-\tilde{1}_{w_{k-1}} \otimes I_{w_{k-2}} & 0 \\
0 & I_{w_{k}} \otimes \tilde{1}_{w_{k-1}}
\end{array}\right], \text { and }
$$

the submatrix in $B_{k}$ restricted to the rows and the columns labelled by the variables in $\mathbf{x}_{k} \uplus \mathbf{x}_{k+1}$ looks like

$$
\left[\begin{array}{cc}
\tilde{1}_{w_{k}} \otimes I_{w_{k-1}} & 0 \\
0 & I_{w_{k+1}} \otimes-\tilde{1}_{w_{k}}
\end{array}\right] .
$$

From Lemma 34, there is a diagonal matrix $D_{k-1}$ in $\mathfrak{g}_{\mathrm{IMM}}$ such that the submatrix restricted to the rows and the columns labelled by the variables in $\mathbf{x}_{k-1} \uplus \mathbf{x}_{k}$ looks like

$$
\left[\begin{array}{cc}
-I_{w_{k-1}} \otimes I_{w_{k-2}} & 0 \\
0 & I_{w_{k}} \otimes I_{w_{k-1}}
\end{array}\right] .
$$

Let $L=B_{k-1}+B_{k}+D_{k-1}$. The submatrix of $L$ restricted to the rows and the columns labelled by the variables in $\mathbf{x}_{k}$ looks as shown in Figure 10.

For notational simplicity we write $w_{k-1}$ as $w$ in Figure 10. If $e_{x}$ is a unit vector in $\mathcal{U}$, where $x=x_{i j}^{(k)}$ is a variable in $\mathbf{x}_{k}$ then the matrix $L$ maps $e_{x}$ to $L e_{x}$ which is the column of $L$ labelled by the variable $x$. This column vector has all entries zero except for the rows labelled by the variables in $\mathbf{x}_{k}$. Restricting to these rows and looking at Figure 10, we infer that the 


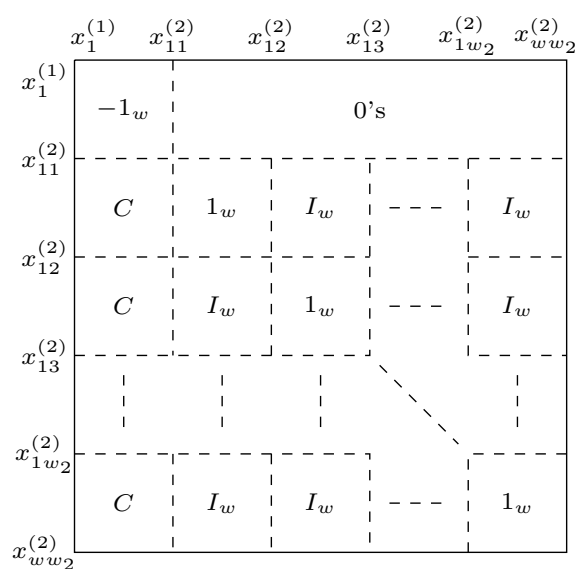

Figure 11 Submatrix of $M$ matrix restricted to rows/columns indexed by $\mathbf{x}_{1} \uplus \mathbf{x}_{2}$.

rows of $L e_{x}$ labelled by the variables $x_{1 j}^{(k)}, x_{2 j}^{(k)}, \ldots, x_{w_{k-1} j}^{(k)}$ are 1 (in particular, these entries are nonzero). We use this knowledge and that $L e_{x} \in \mathcal{U}$ to make the following observation, the proof of which is immediate from Claim 38.

- Observation 66. If $e_{x} \in \mathcal{U}$, where $x=x_{i j}^{(k)}$ then $e_{x^{\prime}} \in \mathcal{U}$ for every $x^{\prime} \in\left\{x_{1 j}^{(k)}, x_{2 j}^{(k)}, \ldots, x_{w_{k-1} j}^{(k)}\right\}$.

Moreover, it follows from the presence of $I_{w}$ matrices in Figure 10 that for every $j^{\prime} \in\left[w_{k}\right]$ there is the variable $y=x_{i j^{\prime}}^{(k)}$ such that the row labelled by $y$ in $L e_{x}$ is 1 , implying ${ }^{40} e_{y} \in \mathcal{U}$. Hence from Observation $66, e_{y^{\prime}} \in \mathcal{U}$ for every $y^{\prime} \in\left\{x_{1 j^{\prime}}^{(k)}, \ldots, x_{w_{k-1} j^{\prime}}^{(k)}\right\}$. Since this is true for every $j^{\prime} \in\left[w_{k}\right], e_{y} \in \mathcal{U}$ for every variable $y \in \mathbf{x}_{k}$ implying $\mathcal{U}=\mathcal{U}_{k}$.

- Claim 41 (restated). The invariant subspaces $\mathcal{U}_{1,2}$ and $\mathcal{U}_{d-1, d}$ are irreducible, and the only invariant subspace properly contained in $\mathcal{U}_{1,2}$ (respectively $\left.\mathcal{U}_{d-1, d}\right)$ is $\mathcal{U}_{2}$ (respectively $\mathcal{U}_{d-1}$ ).

Proof. We prove the claim for $\mathcal{U}_{1,2}$, the proof for $\mathcal{U}_{d-1, d}$ is similar. Suppose $\mathcal{U}_{1,2}=\mathcal{V} \oplus \mathcal{W}$ where $\mathcal{V}, \mathcal{W}$ are invariant subspaces of $\mathfrak{g}_{\mathrm{IM}}$ (and so also coordinate subspaces). A unit vector $e_{x}$, where $x \in \mathbf{x}_{1}$ is either in $\mathcal{V}$ or $\mathcal{W}$. Suppose $e_{x} \in \mathcal{V}$; we will show that $\mathcal{V}=\mathcal{U}_{1,2}$. Without loss of generality, let $x=x_{1}^{(1)}$. Arguing as in the proof of the previous claim, we infer that there is a matrix $M \in \mathfrak{g}_{\mathrm{Mm}}$ such that the submatrix of $M$ restricted to the rows and the columns labelled by the variables in $\mathbf{x}_{1}$ and $\mathbf{x}_{2}$ looks as shown in Figure 11, in which $w=w_{1}$ and $C$ is a $w_{1} \times w_{1}$ anti-symmetric matrix with all non-diagonal entries nonzero. All the other entries of $M$ are zero.

The vector $M e_{x}$ is the first column of $M$ and it is zero everywhere except for the rows labelled by the variables in $\mathbf{x}_{1} \uplus \mathbf{x}_{2}$. Among these rows, unless $y \in\left\{x_{11}^{(2)}, x_{12}^{(2)}, \ldots, x_{1 w_{2}}^{(2)}\right\}$ the row of $M e_{x}$ labelled by $y$ is nonzero. Thus (from Claim 38), $e_{y} \in \mathcal{V}$ for $y \in \mathbf{x}_{1}$ and $y=x_{i j}^{(2)}$ where $i \in\left[2, w_{1}\right]$ and $j \in\left[w_{2}\right]$. Let $y=x_{i j}^{(2)}$ for some $i \in\left[2, w_{1}\right]$ and $j \in\left[w_{2}\right]$. From Figure 11, the row of $M e_{y}$ labelled by $x_{1 j}^{(2)}$ is nonzero and so, for $y^{\prime}=x_{1 j}^{(2)}, e_{y^{\prime}}$ is also in $\mathcal{V}$. Hence, $\mathcal{V}=\mathcal{U}_{1,2}$ and $\mathcal{U}_{1,2}$ is irreducible. To argue that the only invariant subspace properly contained in $\mathcal{U}_{1,2}$ is $\mathcal{U}_{2}$, let $\mathcal{V} \subset \mathcal{U}_{1,2}$ be an invariant subspace of $\mathfrak{g}_{\mathrm{IMM}}$. From the above argument it follows that $e_{x} \notin \mathcal{V}$ for every $x \in \mathbf{x}_{1}$ (otherwise $\mathcal{V}=\mathcal{U}_{1,2}$ ). This implies $\mathcal{V} \subseteq \mathcal{U}_{2}$ and from Claim 40 we have $\mathcal{V}=\mathcal{U}_{2}$

\footnotetext{
${ }^{40}$ Follows again from Claim 38.
} 


\subsection{Proof of claims in Section 4}

- Claim 43 (restated). For all $i \in[s]$, let $\mathcal{N}_{i}$ and $\mathcal{N}_{i}^{\prime}$ be the null spaces of $g_{i}(R)$ and $g_{i}\left(R^{\prime}\right)$. Then

1. $\mathbb{F}^{n}=\mathcal{N}_{1} \oplus \mathcal{N}_{2} \oplus \cdots \oplus \mathcal{N}_{s}=\mathcal{N}_{1}^{\prime} \oplus \mathcal{N}_{2}^{\prime} \oplus \cdots \oplus \mathcal{N}_{s}^{\prime}$.

2. For all $i \in[s], \operatorname{dim}\left(\mathcal{N}_{i}\right)=\operatorname{dim}\left(\mathcal{N}_{i}^{\prime}\right)=\operatorname{deg}_{x}\left(g_{i}\right)$.

Proof. Since $\mathcal{N}_{i}^{\prime}=A^{-1} \mathcal{N}_{i}$ and $A^{-1} \in \mathrm{GL}(n)$, it is sufficient to show $\mathbb{F}^{n}=\mathcal{N}_{1} \oplus \mathcal{N}_{2} \oplus \cdots \oplus \mathcal{N}_{s}$ and $\operatorname{dim}\left(\mathcal{N}_{i}\right)=\operatorname{deg}_{x}\left(g_{i}\right)$. Further, observe that each subspace $\mathcal{N}_{i}$ is non-trivial - if $\mathcal{N}_{1}=\{0\}$ then for all $\mathbf{v} \in \mathbb{F}^{n}, h(R) \cdot \mathbf{v}=g_{1}(R) g_{2}(R) \cdots g_{s}(R) \cdot \mathbf{v}=0$ implying $g_{2}(R) \cdots g_{s}(R) \cdot \mathbf{v}=0$. As the characteristic polynomial and the minimal polynomial have the same irreducible factors this gives a contradiction.

To show the sum of $\mathcal{N}_{i}$ 's is a direct sum it is sufficient to show the following: if $\sum_{l=1}^{s} \mathbf{u}_{l}=0$ where $\mathbf{u}_{l} \in \mathcal{N}_{l}$ then $\mathbf{u}_{l}=0$ for $l \in[s]$. Define for $i \in[s]$

$$
\hat{g}_{i}:=\prod_{j=1, j \neq i}^{s} g_{j}(x)=\frac{h(x)}{g_{i}(x)} .
$$

Since $\hat{g}_{i}(R) \cdot \mathbf{u}_{j}=0$ for $j \neq i$,

$$
\hat{g}_{i}(R) \cdot\left(\sum_{l=1}^{s} \mathbf{u}_{l}\right)=\hat{g}_{i}(R) \cdot \mathbf{u}_{i}=0 .
$$

As $g_{i}(x)$ and $\hat{g}_{i}(x)$ are coprime polynomials, there are $p_{i}(x), q_{i}(x) \in \mathbb{F}[x]$ such that

$$
\begin{aligned}
& p_{i}(x) g_{i}(x)+q_{i}(x) \hat{g}_{i}(x)=1 \Rightarrow p_{i}(R) g_{i}(R)+q_{i}(R) \hat{g}_{i}(R)=I_{n} \\
& \Rightarrow \quad\left(p_{i}(R) g_{i}(R)\right) \cdot \mathbf{u}_{i}+\left(q_{i}(R) \hat{g}_{i}(R)\right) \cdot \mathbf{u}_{i}=\mathbf{u}_{i} .
\end{aligned}
$$

$\operatorname{Both}\left(p_{i}(R) g_{i}(R)\right) \cdot \mathbf{u}_{i}=0\left(\right.$ as $\left.\mathbf{u}_{i} \in \mathcal{N}_{i}\right)$ and $\left(q_{i}(R) \hat{g}_{i}(R)\right) \cdot \mathbf{u}_{i}=0$ (by Equation $(22)$ ). Hence $\mathbf{u}_{i}=0$ for all $i \in[s]$.

Let $\tilde{R}$ be the linear the linear map $R$ restricted to the subspace $\mathcal{N}_{i}$ (this is well defined as $\mathcal{N}_{i}$ is an invariant subspace of $R$ ). Then, $g_{i}(\tilde{R})=0$. Since $g_{i}$ is irreducible, from CayleyHamilton theorem it follows that $g_{i}$ divides the characteristic polynomial of $\tilde{R}$ implying $\operatorname{deg}_{x}\left(g_{i}\right) \leq \operatorname{dim}\left(\mathcal{N}_{i}\right)$. As a consequence, we have

$$
n=\sum_{i=1}^{s} \operatorname{deg}_{x} g_{i} \leq \sum_{i=1}^{s} \operatorname{dim} \mathcal{N}_{i} \leq \operatorname{dim} \mathbb{F}^{n}=n .
$$

Each inequality is an equality, which proves the claim.

- Claim 44 (restated). Suppose $g_{i}(x)$ is an irreducible factor of the characteristic polynomial $h_{k}(x)$ of $R_{k}$ (depicted in Figure 4) for some $k \in[d]$. Then the following holds:

1. If $k \in[2, d-1]$ then $\mathcal{N}_{i} \subseteq \mathcal{U}_{k}$ (equivalently $\mathcal{N}_{i}^{\prime} \subseteq A^{-1} \mathcal{U}_{k}$ ).

2. If $k=1$ then $\mathcal{N}_{i} \subseteq \mathcal{U}_{1,2}$ (equivalently $\mathcal{N}_{i}^{\prime} \subseteq A^{-1} \mathcal{U}_{1,2}$ ), and if $k=d$ then $\mathcal{N}_{i} \subseteq \mathcal{U}_{d-1, d}$ (equivalently $\left.\mathcal{N}_{i}^{\prime} \subseteq A^{-1} \mathcal{U}_{d-1, d}\right)$.

Proof. Figure 12 depicts the matrix $h_{k}(R)$ and as shown in it, call the submatrix restricted to the rows labelled by variables in $\mathbf{x}_{2}$ and columns labelled by variables in $\mathbf{x}_{1} \uplus \mathbf{x}_{2}, M_{k, 2}$; define $M_{k, d-1}$ similarly.

Let $\mathbf{v} \in \mathcal{N}_{i}$. For every $j \in[d]$, let $\mathbf{v}_{j}$ be the subvector of $\mathbf{v}$ restricted to the rows labelled by variables in $\mathbf{x}_{j}$, and $\mathbf{v}_{1,2}$ (respectively $\mathbf{v}_{d-1, d}$ ) be the subvector of $\mathbf{v}$ restricted to the rows 


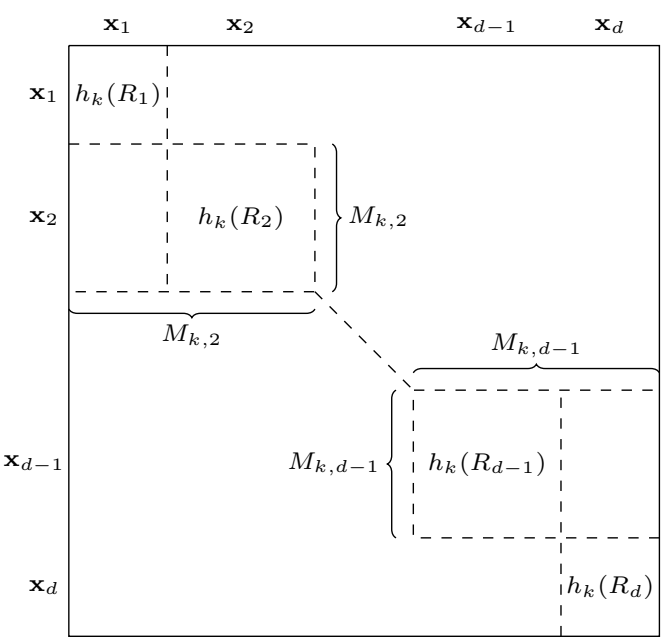

Figure 12 Matrix $h_{k}(R)$.

labelled by variables in $\mathbf{x}_{1} \uplus \mathbf{x}_{2}$ (respectively $\mathbf{x}_{d-1} \uplus \mathbf{x}_{d}$ ). Since $\mathbf{v} \in \mathcal{N}_{i}, g_{i}(R) \cdot \mathbf{v}=0$ implying $h_{k}(R) \cdot \mathbf{v}=0$. Thus we have the following set of equations:

$$
\begin{aligned}
h_{k}\left(R_{1}\right) \cdot \mathbf{v}_{1} & =0 \\
M_{k, 2} \cdot \mathbf{v}_{1,2} & =0 \\
h_{k}\left(R_{j}\right) \cdot \mathbf{v}_{j} & =0 \quad \text { for } j \in[3, d-2] \\
M_{k, d-1} \cdot \mathbf{v}_{d-1, d} & =0 \\
h_{k}\left(R_{d}\right) \cdot \mathbf{v}_{d} & =0 .
\end{aligned}
$$

Case A: $\boldsymbol{k} \in[\mathbf{2}, \boldsymbol{d}-\mathbf{1}]: \quad$ Since $h_{j}(x)$ is the characteristic polynomial of $R_{j}, h_{j}\left(R_{j}\right)=0$ implying $h_{j}\left(R_{j}\right) \cdot \mathbf{v}_{j}=0$ for every $j \in[d]$. As $k \neq 1, h_{k}(x)$ and $h_{1}(x)$ are coprime and from Equation $(24) h_{k}\left(R_{1}\right) \cdot \mathbf{v}_{1}=0$. Hence, $\mathbf{v}_{1}=0$ and for a similar reason $\mathbf{v}_{d}=0$ as $k \neq d$. Thus in Equation (24) we have

$$
\begin{aligned}
& M_{k, 2} \cdot \mathbf{v}_{1,2}=h_{k}\left(R_{2}\right) \cdot \mathbf{v}_{2}=0 \\
& M_{k, d-1} \cdot \mathbf{v}_{d-1, d}=h_{k}\left(R_{d-1}\right) \cdot \mathbf{v}_{d-1}=0 .
\end{aligned}
$$

Therefore for every $j \in[d], h_{k}\left(R_{j}\right) \cdot \mathbf{v}_{j}=0$. If $j \neq k$ then $h_{j}(x)$ and $h_{k}(x)$ are coprime, thus from $h_{j}\left(R_{j}\right) \cdot \mathbf{v}_{j}=0$ we infer $\mathbf{v}_{j}=0$ and hence $\mathbf{v} \in \mathcal{U}_{k}$.

Case B: $\boldsymbol{k}=\mathbf{1}$ or $\boldsymbol{k}=\boldsymbol{d}$ : $\quad$ Let $k=1$, the proof for $k=d$ is similar. Since $h_{k}\left(R_{d}\right) \cdot \mathbf{v}_{d}=0$, $h_{d}\left(R_{d}\right) \cdot \mathbf{v}_{d}=0$, and $h_{k}(x), h_{d}(x)$ are coprime, we get $\mathbf{v}_{d}=0$. Hence from Equation (24),

$$
M_{k, d-1} \cdot \mathbf{v}_{d-1, d}=h_{k}\left(R_{d-1}\right) \cdot \mathbf{v}_{d-1}=0 .
$$

Again for $j \in[3, d], h_{k}\left(R_{j}\right) \cdot \mathbf{v}_{j}=0$ and $h_{j}(x), h_{k}(x)$ are coprime for every $j \neq k$. Hence $\mathbf{v}_{j}=0$ for $j \in[3, d]$ implying $\mathbf{v} \in \mathcal{U}_{1,2}$.

\subsection{Proof of lemma and claim in Section 5}

Lemma 49 (restated). If $f=X_{1} \cdot X_{2} \cdots X_{d}$ and $\mathcal{Y}_{1}, \mathcal{Y}_{1,2}, \mathcal{Y}_{3}, \ldots, \mathcal{Y}_{d-2}, \mathcal{Y}_{d-1, d}, \mathcal{Y}_{d}$ is the output of Algorithm 5 then there is a permutation $\sigma$ on $[3, d-2]$ such that the following hold: 

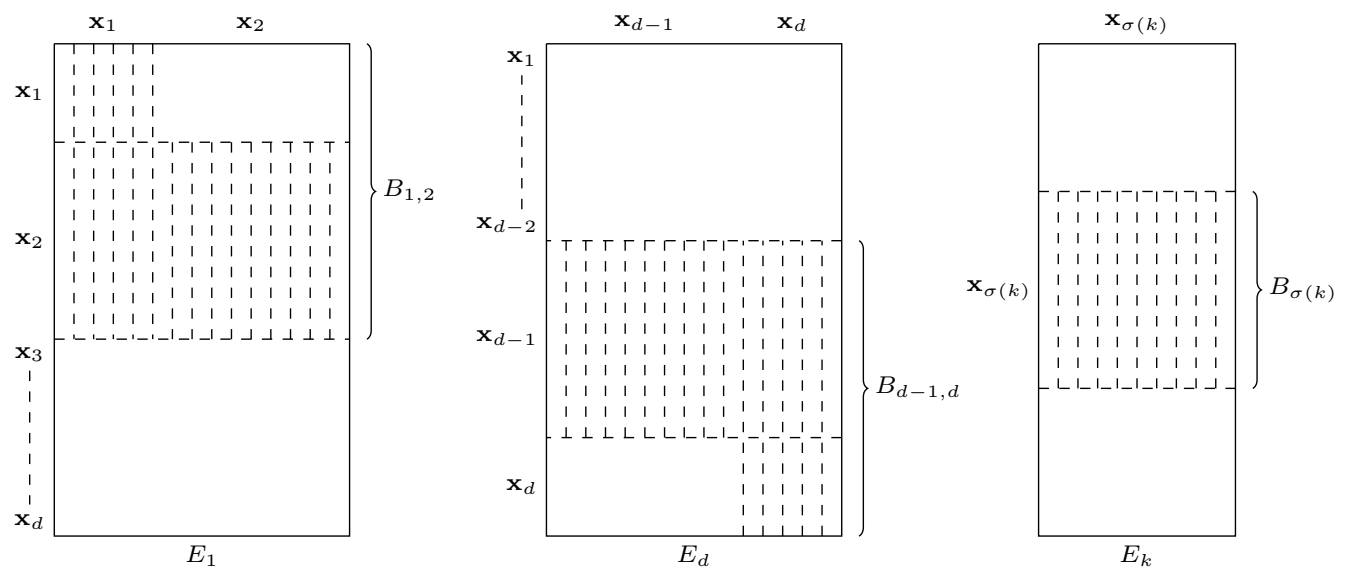

Figure 13 Matrices $E_{1}, E_{d}$ and $E_{k}$.

1. For every $k \in[3, d-2], \mathcal{Y}_{k}=\mathcal{X}_{\sigma(k)}$.

2. Either $\mathcal{Y}_{1}, \mathcal{Y}_{1,2}$ and $\mathcal{Y}_{d}, \mathcal{Y}_{d-1, d}$ are $\mathcal{X}_{1}, \mathcal{X}_{1,2}$ and $\mathcal{X}_{d}, \mathcal{X}_{d-1, d}$ respectively, or $\mathcal{Y}_{1}, \mathcal{Y}_{1,2}$ and $\mathcal{Y}_{d}, \mathcal{Y}_{d-1, d}$ are $\mathcal{X}_{d}, \mathcal{X}_{d-1, d}$ and $\mathcal{X}_{1}, \mathcal{X}_{1,2}$ respectively.

Proof. Assume $\mathcal{V}_{1}$ and $\mathcal{V}_{d}$ are the spaces $A^{-1} \mathcal{U}_{1,2}$ and $A^{-1} \mathcal{U}_{d-1, d}$ respectively. In this case we will show $\mathcal{Y}_{1}, \mathcal{Y}_{1,2}$ and $\mathcal{Y}_{d}, \mathcal{Y}_{d-1, d}$ are $\mathcal{X}_{1}, \mathcal{X}_{1,2}$ and $\mathcal{X}_{d}, \mathcal{X}_{d-1, d}$ respectively ${ }^{41}$. Hence, $u_{1}=w_{1}+w_{1} w_{2}, u_{2}=w_{1} w_{2}, u_{d-1}=w_{d-2} w_{d-1}$ and $u_{d}=w_{d-1}+w_{d-2} w_{d-1}$. From the order of the columns in $V_{1}$ and $V_{d}$ we have $V_{1}=A^{-1} E_{1}$ and $V_{d}=A^{-1} E_{d}$, where $E_{1}$ and $E_{d}$ are $n \times u_{1}$ and $n \times u_{d}$ matrices respectively and they look as shown in Figure 13 .

The rows of $E_{1}$ and $E_{d}$ are labelled by $n$ variables in $\mathbf{x}_{1}$ to $\mathbf{x}_{d}$, whereas the columns of $E_{1}$ are labelled by variables in $\mathbf{x}_{1}$ and $\mathbf{x}_{2}$ and the columns of $E_{d}$ are labelled by variables in $\mathbf{x}_{d-1}$ and $\mathbf{x}_{d}$. Moreover, the nonzero entries in these matrices are restricted to the shaded region in Figure 13.

For $k \in[3, d-2], \mathcal{V}_{k}=A^{-1} \mathcal{U}_{\sigma(k)}$ where $\sigma$ is a permutation on $[3, d-2]$. Hence, $u_{k}=w_{\sigma(k)-1} w_{\sigma(k)}$ and $V_{k}=A^{-1} E_{k}$ where $E_{k}$ is a $n \times u_{k}$ matrix and looks as shown in Figure 13. Again the rows of $E_{k}$ are labelled by the variables $\mathbf{x}_{1}$ to $\mathbf{x}_{d}$, whereas the columns of $E_{k}$ are labelled by variables in $\mathbf{x}_{\sigma(k)}$. The nonzero entries in $E_{k}$ are restricted to the shaded region in Figure 13 whose rows are labelled by variables in $\mathbf{x}_{\sigma(k)}$. Let $E$ be the concatenation of these matrices, $E=\left[E_{1}\left|E_{3}\right| E_{4}|\ldots| E_{d-2} \mid E_{d}\right]$. The rows of $E$ are labelled by $\mathbf{x}_{1}, \mathbf{x}_{2}, \ldots, \mathbf{x}_{d}$ as usual, but now the columns are labelled by $\mathbf{x}_{1}, \mathbf{x}_{2}, \mathbf{x}_{\sigma(3)}$, $\ldots, \mathbf{x}_{\sigma(d-2)}, \mathbf{x}_{d-1}, \mathbf{x}_{d}$ in order as shown in Figure 14. Then $V=A^{-1} E$ implying $V^{-1}=E^{-1} A$. Owing to the structure of $E, E^{-1}$ looks as shown in Figure 14.

The rows of $E^{-1}$ are labelled by $\mathbf{x}_{1}, \mathbf{x}_{2}, \mathbf{x}_{\sigma(3)}, \ldots, \mathbf{x}_{\sigma(d-2)}, \mathbf{x}_{d-1}, \mathbf{x}_{d}$ in order, whereas the columns are labelled by the usual ordering $\mathbf{x}_{1}, \mathbf{x}_{2}, \ldots, \mathbf{x}_{d}$. The submatrix of $E^{-1}$ restricted to the rows and columns labelled by the variables in $\mathbf{x}_{1}$ and $\mathbf{x}_{2}$ is $B_{1,2}^{-1}$ and that labelled by the variables in $\mathbf{x}_{d-1}$ and $\mathbf{x}_{d}$ is $B_{d-1, d}^{-1}$. For $k \in[3, d-2]$ the submatrix restricted to the rows and columns labelled by $\mathbf{x}_{\sigma(k)}$ is $B_{\sigma(k)}^{-1}$. We infer the following facts:

(I) The space spanned by the first $u_{1}-u_{2}$ (that is $w_{1}$ ) rows of $V^{-1}$ is equal to the space spanned by the first $w_{1}$ rows of $A$, the latter space is $\mathcal{X}_{1}$.

${ }^{41}$ If $\mathcal{V}_{1}$ and $\mathcal{V}_{d}$ are the spaces $A^{-1} \mathcal{U}_{d-1, d}$ and $A^{-1} \mathcal{U}_{1,2}$ respectively, then $\mathcal{Y}_{1}, \mathcal{Y}_{1,2}$ and $\mathcal{Y}_{d}, \mathcal{Y}_{d-1, d}$ are $\mathcal{X}_{d}, \mathcal{X}_{d-1, d}$ and $\mathcal{X}_{1}, \mathcal{X}_{1,2}$ respectively - the proof of this case is similar. 

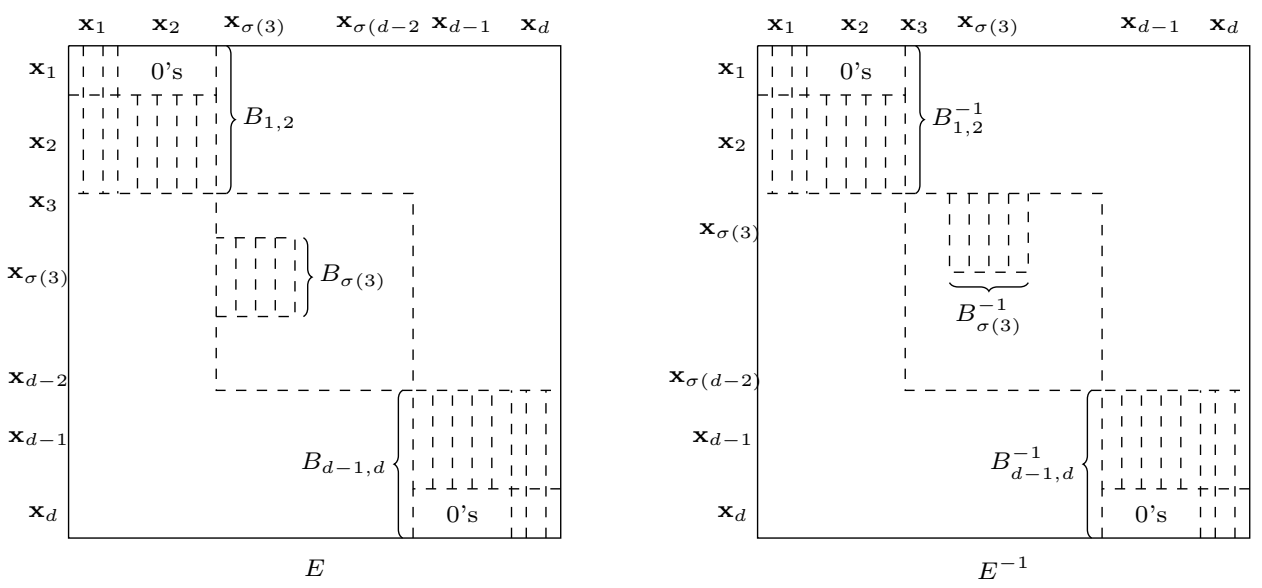

Figure 14 Matrices $E$ and $E^{-1}$.

(II) The space spanned by the first $u_{1}$ (that is $w_{1}+w_{1} w_{2}$ ) rows of $V^{-1}$ is equal to the space spanned by the first $w_{1}+w_{1} w_{2}$ rows of $A$, the latter space is $\mathcal{X}_{1,2}$.

(III) The space spanned by the last $u_{d}$ (that is $w_{d-1}+w_{d-2} w_{d-1}$ ) rows of $V^{-1}$ is equal to the space spanned by the last $w_{d-1}+w_{d-2} w_{d-1}$ rows of $A$, the latter space is $\mathcal{X}_{d-1, d}$.

(IV) The space spanned by the last $u_{d}-u_{d-1}$ (that is $w_{d-1}$ ) rows of $V^{-1}$ is equal to the space spanned by the last $w_{d-1}$ rows of $A$, the latter space is $\mathcal{X}_{d}$.

(V) For $k \in[3, d-2]$ the space spanned by the rows of $V^{-1}$ that are numbered by $t_{k-1}+1$ to $t_{k-1}+u_{k}$ is equal to the space spanned by the rows of $A$ labelled by $\mathbf{x}_{\sigma(k)}$, the latter space is $\mathcal{X}_{\sigma(k)}$.

- Claim 50 (restated). There is a randomized polynomial time algorithm that takes input the bases of the layer spaces $\mathcal{Y}_{1}, \mathcal{Y}_{1,2}, \mathcal{Y}_{3}, \ldots, \mathcal{Y}_{d-2}, \mathcal{Y}_{d-1, d}, \mathcal{Y}_{d}$ and with probability at least $1-\frac{1}{\text { poly }(n)}$ reorders these layer spaces and outputs a width vector $\mathbf{w}^{\prime}$ such that the reordered sequence of spaces and $\mathbf{w}^{\prime}$ are:

1. either $\mathcal{X}_{1}, \mathcal{X}_{1,2}, \mathcal{X}_{3}, \ldots, \mathcal{X}_{d-2}, \mathcal{X}_{d-1, d}, \mathcal{X}_{d}$ and $\left(w_{1}, w_{2}, \ldots, w_{d-1}\right)$ respectively,

2. or $\mathcal{X}_{d}, \mathcal{X}_{d-1, d}, \mathcal{X}_{d-2}, \ldots, \mathcal{X}_{3}, \mathcal{X}_{1,2}, \mathcal{X}_{1}$ and $\left(w_{d}, w_{d-1}, \ldots, w_{1}\right)$ respectively.

Proof. The algorithm employs evaluation dimension to uncover the permutation $\sigma$. Assume that $\mathcal{Y}_{1}, \mathcal{Y}_{1,2}, \mathcal{Y}_{3}, \ldots, \mathcal{Y}_{d-2}, \mathcal{Y}_{d-1, d}, \mathcal{Y}_{d}$ are the spaces $\mathcal{X}_{1}, \mathcal{X}_{1,2}, \mathcal{X}_{\sigma(3)}, \ldots, \mathcal{X}_{\sigma(d-2)}, \mathcal{X}_{d-1, d}, \mathcal{X}_{d}$ respectively ${ }^{42}$. In this case, the algorithm reorders the spaces to a sequence $\mathcal{X}_{1}, \mathcal{X}_{1,2}, \mathcal{X}_{3}, \ldots$, $\mathcal{X}_{d-2}, \mathcal{X}_{d-1, d}, \mathcal{X}_{d}$ and outputs $\mathbf{w}^{\prime}=\mathbf{w}$. For every $k \in[3, d-2]$, let $\mathbf{z}_{k}$ be a set of $\operatorname{dim}\left(\mathcal{Y}_{k}\right)$ many variables. Let $\mathbf{z}_{1}$ (similarly, $\mathbf{z}_{d}$ ) be a set of $\operatorname{dim}\left(\mathcal{Y}_{1}\right)$ (similarly, $\operatorname{dim}\left(\mathcal{Y}_{d}\right)$ ) variables, and let $\mathbf{z}_{2}\left(\operatorname{similarly}, \mathbf{z}_{d-1}\right)$ be a set of $\operatorname{dim}\left(\mathcal{Y}_{1,2}\right)-\operatorname{dim}\left(\mathcal{Y}_{1}\right)\left(\operatorname{similarly}, \operatorname{dim}\left(\mathcal{Y}_{d-1, d}\right)-\operatorname{dim}\left(\mathcal{Y}_{d}\right)\right)$ variables. Finally, let $\mathbf{z}=\mathbf{z}_{1} \uplus \ldots \uplus \mathbf{z}_{d}$ be the set of these $n$ fresh variables.

Compute a linear map $\mu$ that maps $\mathbf{x}$ variables to linear forms in $\mathbf{z}$ variables such that the following conditions are satisfied:

(a) For every $k \in[3, d-2]$, the linear forms corresponding ${ }^{43}$ to the basis vectors of $\mathcal{Y}_{k}$ map to distinct variables in $\mathbf{z}_{k}$.

(b) The linear forms corresponding to the basis vectors in $\mathcal{Y}_{1}$ (similarly, $\mathcal{Y}_{d}$ ) map to distinct variables in $\mathbf{z}_{1}$ ( similarly, $\mathbf{z}_{d}$ ).

\footnotetext{
42 The proof of the other case is similar.

${ }^{43}$ Recall, linear forms in $\mathbf{x}$ variables and vectors in $\mathbb{F}^{n}$ are naturally identified with each other.
} 
(c) The linear forms corresponding to the basis vectors in $\mathcal{Y}_{1,2}$ (similarly, $\mathcal{Y}_{d-1, d}$ ) map to distinct variables in $\mathbf{z}_{1} \uplus \mathbf{z}_{2}$ ( similarly, $\mathbf{z}_{d-1} \uplus \mathbf{z}_{d}$ ).

Conditions (b) and (c) can be simultaneously satisfied as the basis of $\mathcal{Y}_{1}$ (similarly, $\mathcal{Y}_{d}$ ) is contained in the basis of $\mathcal{Y}_{1,2}$ (similarly, $\mathcal{Y}_{d-1, d}$ ) by their very constructions in Algorithm 5. As $f=\mathrm{IMM}_{\mathbf{w}, d}(A \mathbf{x})$, the map $\mu$ takes $f$ to a polynomial $h(\mathbf{z})$ that is computed by a full rank $\mathrm{ABP} \mathrm{A}^{\prime}$ of width $\mathbf{w}$ and length $d$ such that the sets of variables appearing in the $d$ layers of $\mathrm{A}^{\prime}$ from left to right are $\mathbf{z}_{1}, \mathbf{z}_{1} \uplus \mathbf{z}_{2}, \mathbf{z}_{\sigma^{-1}(3)}, \ldots, \mathbf{z}_{\sigma^{-1}(d-2)}, \mathbf{z}_{d-1} \uplus \mathbf{z}_{d}, \mathbf{z}_{d}$ in order.

The following observation, the proof of which is given later, helps find $\sigma^{-1}$ incrementally from blackbox access to $h(\mathbf{z})$. Let $\mathbf{y}_{2}=\mathbf{z}_{1} \uplus \mathbf{z}_{2}$ and $\mathbf{y}_{j}=\mathbf{z}_{1} \uplus \mathbf{z}_{2} \uplus \mathbf{z}_{\sigma^{-1}(3)} \uplus \cdots \uplus \mathbf{z}_{\sigma^{-1}(j)}$, for $j \in[3, d-2]$.

- Observation 67. For every $j \in[2, d-3]$ and $k \in[3, d-2]$ such that $\mathbf{z}_{k} \not \subset \mathbf{y}_{j}$,

1. $\operatorname{Evaldim}_{\mathbf{y}_{j} \uplus \mathbf{z}_{k}}(h)<\left|\mathbf{z}_{k}\right|$, if $k=\sigma^{-1}(j+1)$, and

2. $\operatorname{Evaldim}_{\mathbf{y}_{j} \uplus \mathbf{z}_{k}}(h)>\left|\mathbf{z}_{k}\right|$, if $k \neq \sigma^{-1}(j+1)$.

The proof of the observation also includes an efficient randomized procedure to compute $\operatorname{Evaldim}_{\mathbf{y}_{j} \uplus \mathbf{z}_{k}}(h)$.

Finally, the algorithm outputs the reordered layer spaces $\mathcal{Y}_{1}, \mathcal{Y}_{1,2}, \mathcal{Y}_{\sigma^{-1}(3)}, \ldots, \mathcal{Y}_{\sigma^{-1}(d-2)}$, $\mathcal{Y}_{d-1, d}, \mathcal{Y}_{d}$ which is the ordered sequence of spaces $\mathcal{X}_{1}, \mathcal{X}_{1,2}, \mathcal{X}_{3}, \ldots, \mathcal{X}_{d-2}, \mathcal{X}_{d-1, d}, \mathcal{X}_{d}$. The width vector $\mathbf{w}^{\prime}$ can be readily calculated now by inspecting the dimensions:

$$
\begin{aligned}
w_{1}^{\prime} & =\operatorname{dim}\left(\mathcal{X}_{1}\right)=w_{1}, \\
w_{2}^{\prime} & =\frac{\operatorname{dim}\left(\mathcal{X}_{1,2}\right)-w_{1}}{w_{1}}=w_{2}, \\
w_{k}^{\prime} & =\frac{\operatorname{dim}\left(\mathcal{X}_{k}\right)}{w_{k-1}}=w_{k}, \quad \text { for } k \in[3, d-2], \\
w_{d}^{\prime} & =\operatorname{dim}\left(\mathcal{X}_{d}\right)=w_{d}, \quad \text { and } \\
w_{d-1}^{\prime} & =\frac{\operatorname{dim}\left(\mathcal{X}_{d-1, d}\right)-w_{d}}{w_{d}}=w_{d-1} .
\end{aligned}
$$

This gives $\mathbf{w}^{\prime}=\mathbf{w}$.

Proof of Observation 67. Let $Z_{1} \cdot Z_{2} \cdots Z_{d}$ be equal to $\mathrm{A}^{\prime}$, the full rank ABP of width $\mathbf{w}=\left(w_{1}, w_{2}, \ldots, w_{d-1}\right)$ computing $h$, where the linear forms in $Z_{i}$ are in $\mathbf{z}_{\sigma^{-1}(i)}$ variables for $i \in[3, d-2]$, the linear forms in $Z_{1}, Z_{d}$ are in variables $\mathbf{z}_{1}, \mathbf{z}_{d}$ respectively, and the linear forms in $Z_{2}, Z_{d-1}$ are in $\mathbf{z}_{1} \uplus \mathbf{z}_{2}, \mathbf{z}_{d-1} \uplus \mathbf{z}_{d}$ variables respectively.

Case 1: Suppose $k=\sigma^{-1}(j+1)$, implying $\left|\mathbf{z}_{k}\right|=w_{j} w_{j+1}$. Let $G=Z_{j+2} \cdot Z_{j+3} \cdots Z_{d}$ and the $t$-th entry of $G$ be $g_{t}$ for $t \in\left[w_{j+1}\right]$. As the linear forms in $Z_{1}, Z_{2}, \ldots, Z_{j+1}$ are $\mathbb{F}$-linearly independent, for every $t \in\left[w_{j+1}\right]$ there is a partial evaluation of $h$ at $\mathbf{y}_{j} \uplus \mathbf{z}_{k}$ variables that makes $h$ equal to $g_{t}$. Also, every partial evaluation of $h$ at $\mathbf{y}_{j} \uplus \mathbf{z}_{k}$ variables can be expressed as an $\mathbb{F}$-linear combination of $g_{1}, g_{2}, \ldots, g_{w_{j+1}}$. Hence, from Observation 53 it follows, Evaldim $\mathbf{y}_{j} \uplus \mathbf{z}_{k}(h)=w_{j+1}<\left|\mathbf{z}_{k}\right|$.

Case 2: Suppose $k \neq \sigma^{-1}(j+1)$. The variables $\mathbf{z}_{k}$ appear in the matrix $Z_{\sigma(k)}$, so $\left|\mathbf{z}_{k}\right|=w_{\sigma(k)-1} w_{\sigma(k)}$. Let $G=Z_{\sigma(k)+1} \cdot Z_{\sigma(k)+2} \cdots Z_{d}$ and the $t$-th entry of $G$ be $g_{t}$ for $t \in\left[w_{\sigma(k)}\right]$. Further, let $P=\left(p_{l m}\right)_{l \in\left[w_{j}\right], m \in\left[w_{\sigma(k)-1}\right]}$ be equal to $Z_{j+1} \cdot Z_{j+2} \cdots Z_{\sigma(k)-1}$. As the linear forms in $Z_{1}, Z_{2}, \ldots, Z_{j}$ and $Z_{\sigma(k)}$ are $\mathbb{F}$-linearly independent, there is a partial evaluation of $h$ at the $\mathbf{y}_{j} \uplus \mathbf{z}_{k}$ variables that makes $h$ equal to $p_{l m} g_{t}$ for $l \in\left[w_{j}\right], m \in\left[w_{\sigma(k)-1}\right]$ and $t \in\left[w_{\sigma(k)}\right]$. By Observation 53, $\left\{g_{t} \mid t \in\left[w_{\sigma(k)}\right]\right\}$ are $\mathbb{F}$-linearly independent; using a proof similar to that of Observation 53 we can show that the polynomials $\left\{p_{l m} \mid l \in\right.$ 
$\left.\left[w_{j}\right], m \in\left[w_{\sigma(k)-1}\right]\right\}$ are also $\mathbb{F}$-linearly independent. This implies the set of polynomials $\left\{p_{l m} g_{t} \mid l \in\left[w_{j}\right], m \in\left[w_{\sigma(k)-1}\right]\right.$ and $\left.t \in\left[w_{\sigma(k)}\right]\right\}$ are $\mathbb{F}$-linearly independent, as $p_{l m}$ and $g_{t}$ are on disjoint sets of variables. Since every partial evaluation of $h$ at $\mathbf{y}_{j} \uplus \mathbf{z}_{k}$ variables can be expressed as an $\mathbb{F}$-linear combination of the set of polynomials $\left\{p_{l m} g_{t} \mid l \in\left[w_{j}\right], m \in\right.$ $\left[w_{\sigma(k)-1}\right]$ and $\left.t \in\left[w_{\sigma(k)}\right]\right\}, \operatorname{Evaldim}_{\mathbf{y}_{j} \uplus \mathbf{z}_{k}}(h)=w_{j} w_{\sigma(k)-1} w_{\sigma(k)}=w_{j} \cdot\left|\mathbf{z}_{k}\right|>\left|\mathbf{z}_{k}\right|$.

A randomized procedure to compute Evaldim $\mathbf{y}_{\boldsymbol{j}} \uplus \mathbf{z}_{\boldsymbol{k}}(\boldsymbol{h})$ : Choose evaluation points $\mathbf{a}_{1}, \ldots$, $\mathbf{a}_{n^{2}}$ for the variables $\mathbf{y}_{j} \uplus \mathbf{z}_{k}$ independently and uniformly at random from a set $S^{\left|\mathbf{y}_{j} \uplus \mathbf{z}_{k}\right|} \subset$ $\mathbb{F}^{\left|\mathbf{y}_{j} \uplus \mathbf{z}_{k}\right|}$ with $|S|=\operatorname{poly}(n)$. Output the dimension of the $\mathbb{F}$-linear space spanned by the polynomials $h\left(\mathbf{a}_{1}\right), \ldots, h\left(\mathbf{a}_{n^{2}}\right)$ using Claim 24 .

We argue that the above procedure outputs Evaldim $_{\mathbf{y}_{j} \uplus \mathbf{z}_{k}}(h)$ with probability at least $1-\frac{1}{\operatorname{poly}(n)}$. Let Evaldim $\mathbf{y}_{j} \uplus \mathbf{z}_{k}(h)=e$. Observe that in both Case 1 and $2, e \leq n^{2}$. Also, in both the cases $h$ can be expressed as

$$
h=\sum_{i \in[e]} f_{i}\left(\mathbf{y}_{j} \uplus \mathbf{z}_{k}\right) \cdot q_{i},
$$

where $f_{i}$ and $q_{i}$ are variable disjoint. The polynomials $q_{1}, \ldots, q_{e}$ are the polynomials $g_{1}, \ldots, g_{w_{j+1}}$ in Case 1 ; they are the polynomials $\left\{p_{l m} g_{t} \mid l \in\left[w_{j}\right], m \in\left[w_{\sigma(k)-1}\right]\right.$ and $t \in$ $\left.\left[w_{\sigma(k)}\right]\right\}$ in Case 2. Just as we argue that $q_{1}, \ldots, q_{e}$ are $\mathbb{F}$-linearly independent, we can show that $f_{1}, \ldots, f_{e}$ are also $\mathbb{F}$-linearly independent. So, by Claim 24 the rank of the matrix $M=\left(f_{c}\left(\mathbf{a}_{r}\right)\right)_{r, c \in[e]}$ is $e$ with high probability. This implies the polynomials $h\left(\mathbf{a}_{1}\right), \ldots, h\left(\mathbf{a}_{e}\right)$ are $\mathbb{F}$-linearly independent also with high probability. The correctness of the procedure follows from the observation that the dimension of the $\mathbb{F}$-linear space spanned by $h\left(\mathbf{a}_{1}\right), \ldots, h\left(\mathbf{a}_{n^{2}}\right)$ is upper bounded by $e$ (from Equation (25)).

- Observation 52 (restated). If $h$ is computable by a full rank almost set-multilinear ABP of width $\mathbf{w}$ then there is a full rank almost set-multilinear ABP of width $\mathbf{w}$ in canonical form computing $h$.

Proof. Suppose $X_{1} \cdot X_{2} \cdots X_{d}$ is a full rank almost set-multilinear ABP of width $\mathbf{w}=$ $\left(w_{1}, w_{2}, \ldots, w_{d-1}\right)$ computing $h$. Let $X_{1}^{\prime}=\left(x_{1}^{(1)} x_{2}^{(1)} \ldots x_{w_{1}}^{(1)}\right)$ and $X_{d}^{\prime}=\left(x_{1}^{(d)} x_{2}^{(d)} \ldots x_{w_{d-1}}^{(d)}\right)$. We show there are matrices $X_{2}^{\prime}$ and $X_{d-1}^{\prime}$ satisfying conditions (1b) and (2b) respectively of canonical form (defined in Section 2.4) such that $h=X_{1}^{\prime} \cdot X_{2}^{\prime} \cdot X_{3} \cdots X_{d-2} \cdot X_{d-1}^{\prime} \cdot X_{d}^{\prime}$. We prove the existence of $X_{2}^{\prime}=\left(l_{i j}^{\prime}\right)_{i \in\left[w_{1}\right], j \in\left[w_{2}\right]}$; the proof for $X_{d-1}^{\prime}$ is similar. It is sufficient to show that there is such an $X_{2}^{\prime}$ satisfying $X_{1} \cdot X_{2}=X_{1}^{\prime} \cdot X_{2}^{\prime}$. Denote the $j$-th entry of the $1 \times w_{2}$ matrix $X_{1} \cdot X_{2}$ as $X_{1} \cdot X_{2}(j)$. Similarly $X_{1}^{\prime} \cdot X_{2}^{\prime}(j)$ represents the $j$-th entry of $X_{1}^{\prime} \cdot X_{2}^{\prime}$. Let $g_{i}$ be the sum of all monomials in $X_{1} \cdot X_{2}(j)$ of the following types: $x_{i}^{(1)} x_{k}^{(1)}$ for $k \in\left[i, w_{1}\right]$, and $x_{i}^{(1)} x_{p q}^{(2)}$ for $p \in\left[w_{1}\right], q \in\left[w_{2}\right]$. Clearly,

$$
X_{1} \cdot X_{2}(j)=g_{1}+g_{2}+\cdots+g_{w_{1}} \text {. }
$$

If $l_{i j}^{\prime} \stackrel{\text { def }}{=} g_{i} / x_{i}^{(1)}$ then

$$
X_{1} \cdot X_{2}(j)=x_{1}^{(1)} l_{1 j}^{\prime}+x_{2}^{(1)} l_{2 j}^{\prime}+\cdots+x_{w_{1}}^{(1)} l_{w_{1} j}^{\prime} .
$$

Since $l_{i j}^{\prime}$ is the $(i, j)$-th entry of $X_{2}^{\prime}$, we infer $X_{1} \cdot X_{2}(j)=X_{1}^{\prime} \cdot X_{2}^{\prime}(j)$. By definition, $x_{k}^{(1)}$ does not appear in $l_{i j}^{\prime}$ for $k<i$, and thus condition (1b) is satisfied by $X_{2}^{\prime}$.

- Observation 53 (restated). Let $X_{1} \cdot X_{2} \cdots X_{d}$ be a full rank almost set-multilinear ABP, and $C_{k}=X_{k} \cdots X_{d}$ for $k \in[2, d]$. Let the l-th entry of $C_{k}$ be $h_{k l}$ for $l \in\left[w_{k-1}\right]$. Then the polynomials $\left\{h_{k 1}, h_{k 2}, \cdots, h_{k w_{k-1}}\right\}$ are $\mathbb{F}$-linearly independent. 
Proof. Suppose $\sum_{p=1}^{w_{k-1}} \alpha_{p} \cdot h_{k p}=0$ such that $\alpha_{p} \in \mathbb{F}$ for $p \in\left[w_{k-1}\right]$, and not all $\alpha_{p}=0$. Assume without loss of generality $\alpha_{1} \neq 0$. Since the linear forms in $X_{k}, \ldots, X_{d}$ are $\mathbb{F}$-linearly independent, there is an evaluation of the variables in $\mathbf{x}_{k} \uplus \cdots \uplus \mathbf{x}_{d}$ to field constants such that $h_{k 1}=1$ and every other $h_{k p}=0$ under this evaluation. This implies $\alpha_{1}=0$, contradicting our assumption.

\subsection{Proof of observation in Section 6}

- Observation 57 (restated). There are matrices $A_{1}, \ldots, A_{d-1}$ with $A_{k} \in \mathrm{GL}\left(w_{k}\right)$ for every $k \in[d-1]$, such that $X_{1}=Q_{1} \cdot A_{1}, X_{2}\left(\mathbf{x}_{2}\right)=A_{1}^{-1} \cdot Q_{2} \cdot A_{2}, X_{d-1}\left(\mathbf{x}_{d-1}\right)=A_{d-2}^{-1} \cdot Q_{d-1} \cdot A_{d-1}$, $X_{d}=A_{d-1}^{-1} \cdot Q_{d}$, and $X_{k}=A_{k-1}^{-1} \cdot Q_{k} \cdot A_{k}$ for $k \in[3, d-2]$.

Proof. To simplify notations, we write $X_{2}\left(\mathbf{x}_{2}\right), X_{d-1}\left(\mathbf{x}_{d-1}\right)$ as $X_{2}, X_{d-1}$ respectively. We have

$$
X_{1} \cdot X_{2} \cdots X_{d-1} \cdot X_{d}=Q_{1} \cdot Q_{2} \cdots Q_{d-1} \cdot Q_{d}=\mathrm{IMM}
$$

where the dimensions of the matrices $X_{k}$ and $Q_{k}$ are the same, and the set of variables appearing in both $X_{k}$ and $Q_{k}$ is $\mathbf{x}_{k}$, for every $k \in[d]$. Since the linear forms in $X_{1}$ are $\mathbb{F}$-linearly independent, there is an $A_{1} \in \mathrm{GL}\left(w_{1}\right)$ such that $X_{1}=Q_{1} \cdot A_{1}$, implying

$$
\begin{aligned}
& Q_{1} \cdot\left[A_{1} \cdot X_{2} \cdots X_{d-1} \cdot X_{d}-Q_{2} \cdots Q_{d-1} \cdot Q_{d}\right]=0 \\
\Rightarrow \quad & X_{2} \cdots X_{d-1} \cdot X_{d}=A_{1}^{-1} \cdot Q_{2} \cdots Q_{d-1} \cdot Q_{d},
\end{aligned}
$$

as the formal variable entries of $Q_{1}$ do not appear in the matrices $X_{k}, Q_{k}$ for $k \in[2, d]$. The rest of the proof proceeds inductively: Suppose for some $k \in[2, d-1]$,

$$
X_{k} \cdots X_{d-1} \cdot X_{d}=A_{k-1}^{-1} \cdot Q_{k} \cdots Q_{d-1} \cdot Q_{d}, \quad \text { where } A_{k-1} \in \mathrm{GL}\left(w_{k-1}\right) .
$$

Let $p_{k}=\sum_{i=k+1}^{d}\left|\mathbf{x}_{i}\right|$. Since the linear forms in $X_{k+1}, \ldots, X_{d-1}, X_{d}$ are $\mathbb{F}$-linearly independent, for every $l \in\left[w_{k}\right]$ there is a point $\mathbf{a}_{l} \in \mathbb{F}^{p_{k}}$ such that the $w_{k} \times 1$ matrix $X_{k+1} \cdots X_{d-1} \cdot X_{d}$ evaluated at $\mathbf{a}_{l}$ has 1 at the $l$-th position and all its other entries are zero. Let $A_{k}$ be the $w_{k} \times w_{k}$ matrix such that the $l$-th column of $A_{k}$ is equal to $Q_{k+1} \cdots Q_{d-1} \cdot Q_{d}$ evaluated at $\mathbf{a}_{l}$. Then, $X_{k}=A_{k-1}^{-1} \cdot Q_{k} \cdot A_{k}$. As the linear forms in $X_{k}$ and $Q_{k}$ are $\mathbb{F}$-linearly independent, it must be that $A_{k} \in \mathrm{GL}\left(w_{k}\right)$. Putting this expression for $X_{k}$ in the equation above and arguing as before, we get a similar equation with $k$ replaced by $k+1$. The proof then follows by induction.

\section{References}

1 Scott Aaronson. Arithmetic natural proofs theory is sought. http://www. scottaaronson. $\mathrm{com} / \mathrm{blog} / \mathrm{p}=336,2008$.

2 Manindra Agrawal. Proving Lower Bounds Via Pseudo-random Generators. In FSTTCS 2005: Foundations of Software Technology and Theoretical Computer Science, 25th International Conference, Hyderabad, India, December 15-18, 2005, Proceedings, pages 92-105, 2005.

3 Manindra Agrawal, Rohit Gurjar, Arpita Korwar, and Nitin Saxena. Hitting-Sets for ROABP and Sum of Set-Multilinear Circuits. SIAM J. Comput., 44(3):669-697, 2015.

4 Manindra Agrawal and Nitin Saxena. Equivalence of f-algebras and cubic forms. In STACS 2006, 23rd Annual Symposium on Theoretical Aspects of Computer Science, Marseille, France, February 23-25, 2006, Proceedings, pages 115-126, 2006.

5 Dana Angluin. Queries and concept learning. Machine Learning., 2(4):319-342, 1988. 
6 Vikraman Arvind, Partha Mukhopadhyay, and Srikanth Srinivasan. New Results on Noncommutative and Commutative Polynomial Identity Testing. In Proceedings of the 23rd Annual IEEE Conference on Computational Complexity, CCC 2008, 23-26 June 2008, College Park, Maryland, USA, pages 268-279, 2008.

7 Amos Beimel, Francesco Bergadano, Nader H. Bshouty, Eyal Kushilevitz, and Stefano Varricchio. Learning functions represented as multiplicity automata. J. ACM, 47(3):506$530,2000$.

8 Michael Ben-Or and Richard Cleve. Computing algebraic formulas using a constant number of registers. In Proceedings of the 20th Annual ACM Symposium on Theory of Computing, May 2-4, 1988, Chicago, Illinois, USA, pages 254-257, 1988.

9 Elwyn Berlekamp. Factoring polynomials over finite fields. Bell System Technical Journal, 46:1853-1859, 1967.

10 Lenore Blum, Mike Shub, and Steve Smale. On a Theory of Computation over the Real Numbers; NP Completeness, Recursive Functions and Universal Machines (Extended Abstract). In 29th Annual Symposium on Foundations of Computer Science, White Plains, New York, USA, 24-26 October 1988, pages 387-397, 1988.

11 David G. Cantor and Hans Zassenhaus. A new algorithm for factoring polynomials over finite fields. Mathematics of Computation, 36:587-592, 1981.

12 Enrico Carlini. Reducing the number of variables of a polynomial. In Algebraic geometry and geometric modelling, Mathematics and Visualization, Springer, pages 237-247, 2006.

13 Zeev Dvir, Rafael Mendes de Oliveira, and Amir Shpilka. Testing equivalence of polynomials under shifts. In Automata, Languages, and Programming - 41st International Colloquium, ICALP 2014, Copenhagen, Denmark, July 8-11, 2014, Proceedings, Part I, pages 417-428, 2014.

14 Michael A. Forbes and Amir Shpilka. Quasipolynomial-time identity testing of noncommutative and read-once oblivious algebraic branching programs. In 54th Annual IEEE Symposium on Foundations of Computer Science, FOCS 2013, 26-29 October, 2013, Berkeley, CA, USA, pages 243-252, 2013.

15 Fulvio Gesmundo. Gemetric aspects of iterated matrix multiplication. Journal of Algebra, 461:42-64, 2016.

16 Joshua A. Grochow. Symmetry and equivalence relations in classical and geometric complexity theory. PhD thesis, The University of Chicago, 2012.

17 Ankit Gupta, Neeraj Kayal, and Satyanarayana V. Lokam. Efficient Reconstruction of Random Multilinear Formulas. In IEEE 52nd Annual Symposium on Foundations of Computer Science, FOCS 2011, Palm Springs, CA, USA, October 22-25, 2011, pages 778-787, 2011.

18 Ankit Gupta, Neeraj Kayal, and Satyanarayana V. Lokam. Reconstruction of depth-4 multilinear circuits with top fan-in 2. In Proceedings of the 44th Symposium on Theory of Computing Conference, STOC 2012, New York, NY, USA, May 19-22, 2012, pages 625-642, 2012.

19 Ankit Gupta, Neeraj Kayal, and Youming Qiao. Random Arithmetic Formulas Can Be Reconstructed Efficiently. In Proceedings of the 28th Conference on Computational Complexity, CCC 2013, K.lo Alto, California, USA, 5-7 June, 2013, pages 1-9, 2013.

20 Johan Håstad. Tensor Rank is NP-Complete. In Automata, Languages and Programming, 16th International Colloquium, ICALP89, Stresa, Italy, July 11-15, 1989, Proceedings, pages 451-460, 1989.

21 Joos Heintz and Claus-Peter Schnorr. Testing Polynomials which Are Easy to Compute (Extended Abstract). In Proceedings of the 12th Annual ACM Symposium on Theory of Computing, April 28-30, 1980, Los Angeles, California, USA, pages 262-272, 1980. 
22 Erich Kaltofen and Barry M. Trager. Computing with Polynomials Given By Black Boxes for Their Evaluation: Greatest Common Divisors, Factorization, Separation of Numerators and Denominators. In 29th Annual Symposium on Foundations of Computer Science, White Plains, New York, USA, 24-26 October 1988, pages 296-305, 1988.

23 Zohar Shay Karnin and Amir Shpilka. Reconstruction of generalized depth-3 arithmetic circuits with bounded top fan-in. In Proceedings of the 24th Annual IEEE Conference on Computational Complexity, CCC 2009, Paris, France, 15-18 July 2009, pages 274-285, 2009.

24 Neeraj Kayal. Efficient algorithms for some special cases of the polynomial equivalence problem. In Proceedings of the Twenty-Second Annual ACM-SIAM Symposium on Discrete Algorithms, SODA 2011, San Francisco, California, USA, January 23-25, 2011, pages 1409-1421, 2011.

25 Neeraj Kayal. Affine projections of polynomials: extended abstract. In Proceedings of the 44th Symposium on Theory of Computing Conference, STOC 2012, New York, NY, USA, May 19-22, 2012, pages 643-662, 2012.

26 Neeraj Kayal. An exponential lower bound for the sum of powers of bounded degree polynomials. Electronic Colloquium on Computational Complexity (ECCC), 19:81, 2012.

27 Neerak Kayal, Chandan Saha, and Sébastien Tavenas. An Average-Case Matrix Factorization Problem. Work in progress, 2017.

28 Adam Klivans, Pravesh Kothari, and Igor Carboni Oliveira. Constructing Hard Functions Using Learning Algorithms. In Proceedings of the 28th Conference on Computational Complexity, CCC 2013, K.lo Alto, California, USA, 5-7 June, 2013, pages 86-97, 2013.

29 Adam Klivans and Amir Shpilka. Learning arithmetic circuits via partial derivatives. In Computational Learning Theory and Kernel Machines, 16th Annual Conference on Computational Learning Theory and 7th Kernel Workshop, COLT/Kernel 2003, Washington, DC, USA, August 24-27, 2003, Proceedings, pages 463-476, 2003.

30 Adam Klivans and Daniel A. Spielman. Randomness efficient identity testing of multivariate polynomials. In Proceedings on 33rd Annual ACM Symposium on Theory of Computing, July 6-8, 2001, Heraklion, Crete, Greece, pages 216-223, 2001.

31 A.K. Lenstra, H.W.jun. Lenstra, and Lászlo Lovász. Factoring polynomials with rational coefficients. Math. Ann., 261:515-534, 1982.

32 Meena Mahajan and V. Vinay. Determinant: Combinatorics, algorithms, and complexity. Chicago J. Theor. Comput. Sci., 1997, 1997.

33 Daniel Minahan and Ilya Volkovich. Complete Derandomization of Identity Testing and Reconstruction of Read-Once Formulas. Electronic Colloquium on Computational Complexity (ECCC), 23:171, 2016.

34 Noam Nisan. Lower bounds for non-commutative computation (extended abstract). In Proceedings of the 23rd Annual ACM Symposium on Theory of Computing, May 5-8, 1991, New Orleans, Louisiana, USA, pages 410-418, 1991.

35 Jacques Patarin. Hidden fields equations (HFE) and isomorphisms of polynomials (IP): two new families of asymmetric algorithms. In Advances in Cryptology - EUROCRYPT'96, International Conference on the Theory and Application of Cryptographic Techniques, Saragossa, Spain, May 12-16, 1996, Proceeding, pages 33-48, 1996.

36 Alexander A. Razborov and Steven Rudich. Natural proofs. In Proceedings of the TwentySixth Annual ACM Symposium on Theory of Computing, 23-25 May 1994, Montréal, Québec, Canada, pages 204-213, 1994.

37 Amir Shpilka. Interpolation of depth-3 arithmetic circuits with two multiplication gates. In Proceedings of the 39th Annual ACM Symposium on Theory of Computing, San Diego, California, USA, June 11-13, 200\%, pages 284-293, 2007. 
38 Amir Shpilka and Ilya Volkovich. Improved Polynomial Identity Testing for Read-Once Formulas. In Approximation, Randomization, and Combinatorial Optimization. Algorithms and Techniques, 12th International Workshop, APPROX 2009, and 13th International Workshop, RANDOM 2009, Berkeley, CA, USA, August 21-23, 2009. Proceedings, pages 700-713, 2009.

39 Amir Shpilka and Amir Yehudayoff. Arithmetic circuits: A survey of recent results and open questions. Foundations and Trends in Theoretical Computer Science, 5(3-4):207-388, 2010.

40 Gaurav Sinha. Reconstruction of real depth-3 circuits with top fan-in 2. In 31st Conference on Computational Complexity, CCC 2016, May 29 to June 1, 2016, Tokyo, Japan, pages 31:1-31:53, 2016.

41 Thomas Thierauf. The isomorphism problem for read-once branching programs and arithmetic circuits. Chicago J. Theor. Comput. Sci., 1998, 1998.

42 Ilya Volkovich. A guide to learning arithmetic circuits. In Proceedings of the 29th Conference on Learning Theory, COLT 2016, New York, USA, June 23-26, 2016, pages 1540-1561, 2016. 